2. To: (Receiving Organization)

TWRS Equipment Engineering

5. Proj./Prog./Dept./Div.:

Stabilization

8. Originator Remarks:

Release of HNF-3776, rev. 0.

USQ\# TF-98-0466 REVIO
11. Receiver Remarks: 11A. Design Baseline Document? [X] Yes [] No
3. From: (Originating Organization)

B. E. Bielicki, FDNW

6. Design Authority/ Design Agent/Cog. Engr.:

J. R. Kriskovich
4. Related EDT No.:

N/A

7. Purchase Order No.:

$$
\text { N/A }
$$

9. Equip./Component No.:

500 CFM Portable Exhausters

10. System/Blog./Facility: TWRS-200G

12. Major Assm. Dwg. No.:

H-14-100867

13. Permit/Permit Application No.: $N / A$

14. Required Response Date:

ASAP

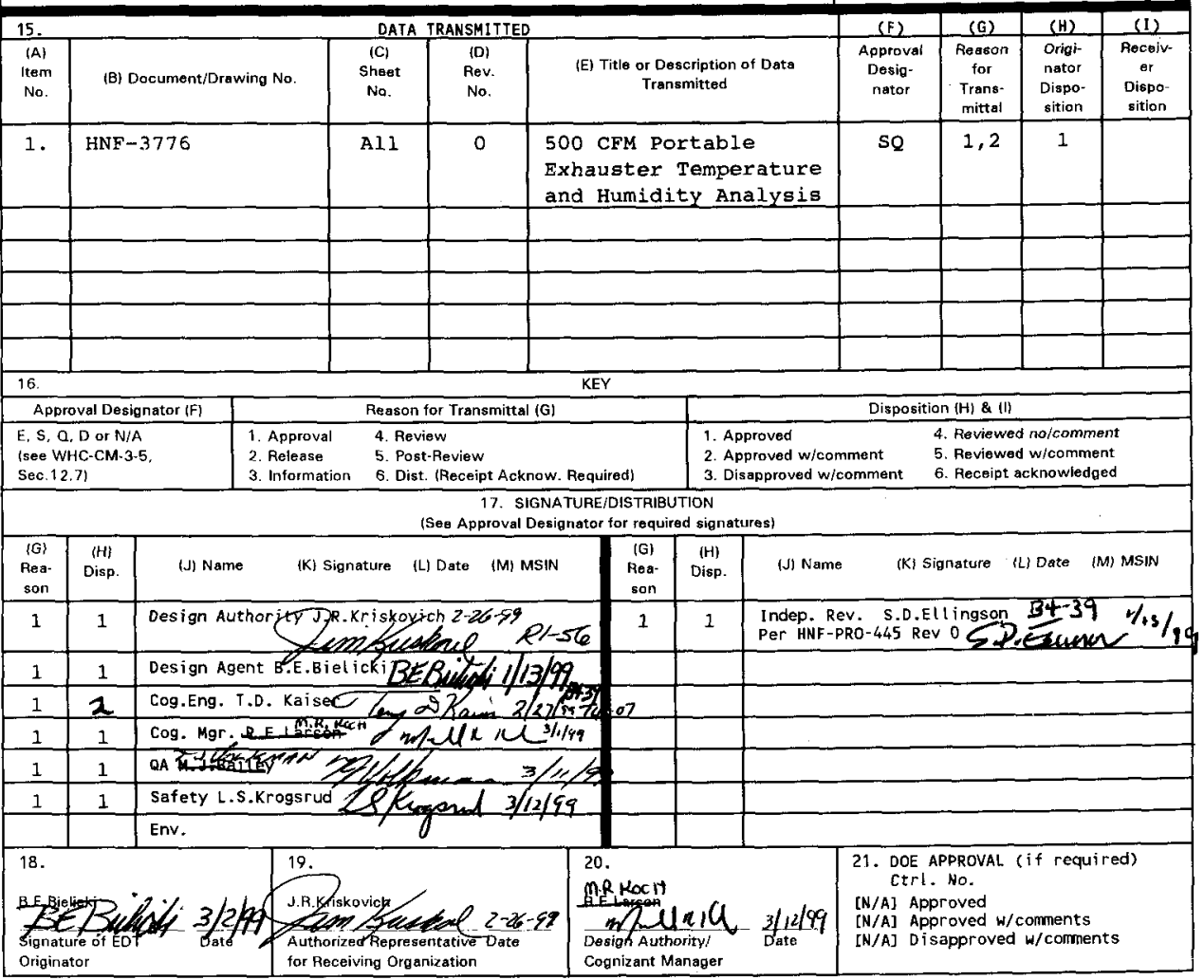




\title{
500 CFM Portable Exhauster Temperature and Humidity Analysis
}

\author{
B. B. Bielicki \\ Fluor Daniel Northwegt, Richland, WA 99352 \\ U.S. Department of Energy Contract DE-ACO6-96RL13200 \\ EDT/ECN : EDT-620994 \\ UC: 2030 \\ Org Code: 454 \\ B\&R Code: EW3120072 \\ Charge Code: 103360 EFoO \\ Total Pages: 96 \\ Key Words: Portable Exhauster, GEMS, skid, Saltwells, POR04, POR05, \\ POR06 \\ Abstract: Condensation must be prevented from collecting on the 500 CFM \\ portable exhauster filters. To ensure the air stream is above the dew \\ point, it will be heated. The purpose of this document is to determine \\ the maximum temperature and water content of the air stream and how much \\ it should be heated to prevent condensation formation problems. \\ Additionally, the maximum possible temperature the continuous air \\ monitor (CAM) head will be exposed to will be compared to the allowable.
}

TRADEMARK DISCLAIMER. Reference herein to any specific comercial product, process, or service by trade name, trademark, manufacturer, or otherwise, does not necessarily constitute or imply its endorsement, recommendation, or favoring by the United States Government or any agency thereof or $i$ ts contractors or subcontractors.

Printed in the United states of America. To obtain copies of this document, contact: Document Control Services, P.O. Box 950. Mailstop H6-08, Richland WA 99352, Phone (509) 372-2420; Fax (509) 376-4989.
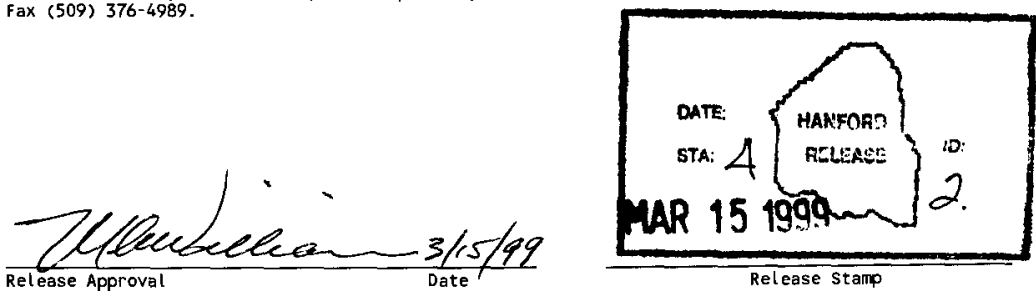

Release stamp 
HNF-3776, Rev. 0

Page 1 of 95

\section{BACKGROUND}

$500 \mathrm{cfm}$ portable exhausters will be utilized on single shell tanks involved in saltwell pumping. This will be done, in part, to remove flammable gases from the tank vapor space. The exhaust filter train, fan, stack, and associated instrumentation and equipment are mounted on a portable skid. The design analysis and basis for the skid system design are documented in reference 1 .

A pumped drainage collection system is being added to the existing portable exhausters. Additional equipment and instrumentation are also being added to the exhausters, including a vacuum pump cabinet and a generic effluent monitoring system (GEMS). The GEMS will provide sampling and monitoring capabilities.

\section{PURPOSE}

The purpose of this analysis is three fold. First, to determine the maximum saltwell tank vapor space temperature. Second, to determine an allowable exhauster inlet air temperature increase to ensure the humidity is less than $70 \%$. Third, to assess potential adverse temperature effects to the continuous air monitor (CAM) sample head.

The results of this analysis will be used to ensure that air stream temperatures in the portable exhausters are increased sufficiently to prevent condensation from forming on either the pre or HEPA filters without adversely effecting the CAM.

\section{ANALYSIS APPROACH}

The following will be determined in this analysis:

I. Determine the Maximum Saltwell Tank Vapor Space Temperature

II. Determine a Reasonable Exhauster Inlet Air Temperature Increase

III. Evaluate Air Temperature Relative to CAM Sample Head Operation

\section{References}

1. Design Calculations and Methods for $500 \mathrm{cfm}$ Portable Exhauster VTP-2212A, HNF-SD-WM-DA-230, rev. 0.

2. H-14-100867

3. ASHRAE Fundamentals Handbook, 1993 edition.

4. Functional Requirements for Portable Exhauster Systems to Be Used During Saltwell Pumping, HNF-SD-WM-DB-035 Rev. 2. 
HNF-3776, Rev. 0

Page 2 of 95

\section{ASSUMPTIONS AND BASIS}

1. The tank vapor space evaluation covers October 1995 thru October 1998.

2. The humidity of the air stream must be equal to or less than $70 \%$ relative humidity after being heated.

3. The maximum exhauster inlet temperature from any saltwell tank, prior to heating, is 120 degrees Fahrenheit at $100 \%$ relative humidity (see ref. 4).

4. None of the saltwell tanks will receive higher activity (thermally hotter) waste.

5. The data used as a basis for this report originates from the Surveillance Analysis Computer System (SACS). This data is the most defensible and up-to-date information available.

\section{SUMMARY OF KEY FINDINGS}

1. The maximum inlet exhauster temperature is conservatively 120 degrees Fahrenheit.

2. Increasing the air stream temperature a maximum of 20 degrees Fahrenheit will prevent condensation.

3. The CAM will not be adversely effected by sample stream temperatures.

\section{ANALYSIS}

\section{Determine the Maximum Saltwell Tank Vapor Space Temperature}

To determine the vapor space temperatures, a list of the saltwell tanks was first developed. The portable exhauster has the potential of being utilized on any of the saltwell tanks. Then tank liquid levels were determined and compared with thermocouple locations. With this information, vapor space temperatures were then determined and plotted. The summary of the temperature results for each of the saltwell tanks and the SACS data from which these temperatures were determined are attached.

With the exception of two temperature spikes, which appear to be anomalies, the minimum and maximum temperature range which bounds all the tanks is 71 to 107 degrees Fahrenheit. The maximum temperature spike was 115 in tank A-101. For conservatism, the value of 120 degrees Fahrenheit will be used as the maximum exhauster inlet temperature at $100 \%$ relative humidity. This is also the maximum temperature given in reference 4 . Using the maximum temperature as the basis for the analysis will ensure that the highest amount of moisture in the air stream is accounted for. 
HNF-3776, Rev. 0

Page 3 of 95

\section{Determine a Reasonable Exhauster Inlet Air Temperature Increase}

The approach will be to assess a 20 degree inlet air temperature increase and evaluate it's reasonableness.

$\phi=P_{v} / P_{a}($ Ref. 3)

\section{Where}

$\phi=\mathbf{R H}=$ relative humidity

$\mathrm{P}_{\mathrm{v}}=$ partial pressure of the vapor in the actual gas mixture, inches $\mathrm{Hg}$, absolute

$\mathrm{P}_{\mathrm{a}}=$ partial pressure of the vapor in a saturated gas mixture, inches $\mathrm{Hg}$, absolute

At 120 degrees $\mathrm{F}$ and $100 \% \mathrm{RH}, \mathrm{P}_{\mathrm{a}}=\mathrm{P}_{\mathrm{v}}=3.45052$ in. $\mathrm{Hg}$ (Ref. 3)

At 140 degrees $\mathrm{F}$ and $100 \% \mathrm{RH}$, the partial pressure of water is $\mathrm{P}_{\mathrm{a}}=5,88939$ in. $\mathrm{Hg}$ (Ref. 3)

The inlet and outlet mass is the same. Therefore:

$\phi=3.45052$ in. $\mathrm{Hg} . / 5.88939 \mathrm{in} . \mathrm{Hg}=.586$

In percent, $\phi=58.6 \%<70 \%$

As a check, the alternative approach below will be used.

$\phi=\mathrm{n}_{\mathrm{v}} / \mathrm{n}_{\mathrm{a}}$ (Ref. 3)

Where

$\phi=\mathrm{RH}=$ relative humidity

$\mathrm{n}_{v}=$ mole fraction of the vapor in the mixture, constant at $120 \mathrm{~F}$ and $140 \mathrm{~F}$

$n_{a}=$ mole fraction of the vapor in a saturated mixture

$1 \mathrm{lb}$ dry air (da) $x 1 \mathrm{lbmole} / 28,96 \mathrm{lb} \mathrm{da}=.034530 \mathrm{lbmole}$ da

At $120 \mathrm{~F}, \mathrm{~W}=$ humidity ratio $=.081560 \mathrm{lb}$ water $/ \mathrm{lb} \mathrm{da}$

$.081560 \mathrm{lb}$ water $\mathrm{x} 1 \mathrm{lbmole} / 18 \mathrm{lb}$ water $=.004531 \mathrm{lbmole}$ water

$\mathrm{n}_{\mathrm{v}}=.004531 \mathrm{lbmole}$ water $/(.004531 \mathrm{lbmole}$ water $+.034530 \mathrm{lbmole} \mathrm{da})=.115998$ 
HNF-3776, Rev. 0

Page 4 of 95

At $140 \mathrm{~F}, \mathrm{~W}=$ humidity ratio $=.153538 \mathrm{lb}$ water $/ \mathrm{b} \mathrm{da}$

$.153538 \mathrm{lb}$ water $\mathrm{x} 1 \mathrm{lbmole} / 18 \mathrm{lb}$ water $=.008530 \mathrm{lbmole}$ water

$\mathrm{n}_{\mathrm{a}}=.008530 \mathrm{lbmole}$ water $/(.008530 \mathrm{lbmole}$ water $+.03453 \mathrm{lbmole} \mathrm{da})=.198094$

$\phi=100\left(\mathrm{n}_{\mathrm{v}} / \mathrm{n}_{\mathrm{a}}\right)=100(.115998 / .198094)=58.6 \%<70 \%$

Heating the exhauster inlet air 20 degrees $\mathrm{F}$ exceeds the design requirements.

\section{Evaluate Air Temperature Relative to (CAM) Sample Head Operation}

Eberline vendor information states that the CAM sample head can operate at a temperature up to 140 Fahrenheit without degradation or adversely effecting monitoring readings. Because the maximum exhauster inlet temperature has been conservatively taken as 120 degrees Fahrenheit, the maximum it can be internally heated to is 140 degrees Fahrenheit. Additionally, the air stream can also experience heat loss or heat gain as it is routed from the inlet, through the filtration bank, fan, stack (to the point where the sample is taken), and thru the sample lines, depending on the ambient conditions. However, the residence time of the air stream in the exhaust system is very short, making temperature changes due to external influences insignificant. Therefore the temperature is not expected to exceed the 140 degree maximum, protecting the sample head CAM from adverse effects. 
HNF-3776, Rev. 0

Page 5 of 95

\begin{tabular}{|c|c|c|c|}
\hline \multicolumn{4}{|c|}{$\begin{array}{l}500 \text { CFM PORTABLE EXHAUSTER TANK VAPOR SPACE TEMPERATURE } \\
\text { BASIS DATA SUMMARY }\end{array}$} \\
\hline \multicolumn{4}{|c|}{ Note: Basis Data from the Surveillance Analysis Computer System (SACS) } \\
\hline $\begin{array}{l}\text { TANK } \\
\text { NUMBER }\end{array}$ & $\begin{array}{l}\text { MAX VAPOR SPACE } \\
\text { TEMP IN LAST } 3 \text { YRS, } \\
\text { FAHRENHEIT }\end{array}$ & $\begin{array}{l}\text { TEMP SPIKES JUDGED } \\
\text { TO BE ANOMALIES, } \\
\text { FAHRENHEIT }\end{array}$ & COMMENTS \\
\hline & & & \\
\hline $\mathrm{A}-101$ & 106 & $1 @ 115$ & \\
\hline$A X-101$ & 95 & & \\
\hline BY-105 & 88 & & \\
\hline BY-106 & 89 & & \\
\hline C-103 & 107 & & \\
\hline S-101 & 97 & $1 @ 102$ & \\
\hline S-102 & 86 & & \\
\hline S-103 & 81 & & \\
\hline S-106 & 78 & & \\
\hline S-107 & 89 & & \\
\hline S-109 & 84 & & \\
\hline S-111 & 81 & & \\
\hline $\mathrm{S}-112$ & 79 & & \\
\hline$S x-101$ & 107 & & \\
\hline$S X-102$ & 99 & & \\
\hline $5 x-103$ & 98 & & \\
\hline$S X-104$ & 100 & & \\
\hline $5 x-105$ & 100 & & \\
\hline $5 X-106$ & 93 & & TC in top of liq. \\
\hline$T-104$ & 73 & & \\
\hline$T-110$ & 71 & & \\
\hline$U-102$ & 87 & & \\
\hline$U-103$ & 87 & & \\
\hline U-105 & 88 & & \\
\hline U-106 & 83 & & \\
\hline U-107 & 79 & & \\
\hline U-108 & 82 & & \\
\hline U-109 & 81 & & \\
\hline $\mathrm{U}-111$ & 80 & & \\
\hline
\end{tabular}

The Surveillance Analysis Computer System (SACS) data used as input for the above table follows. 
HNF-3776 REV. O PG. 6 OF 95

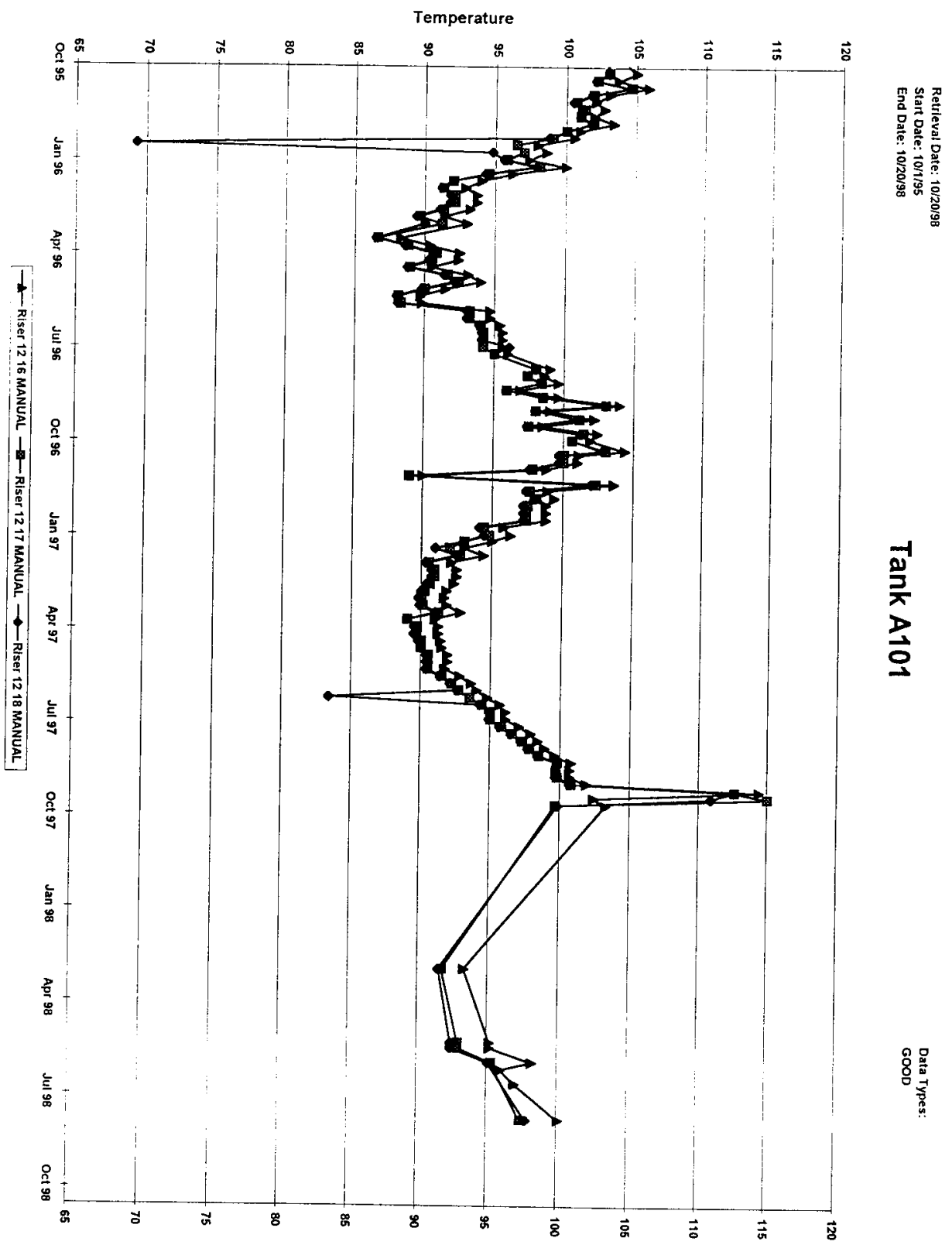


HNF-3776 REV. O PG. 7 OF 95

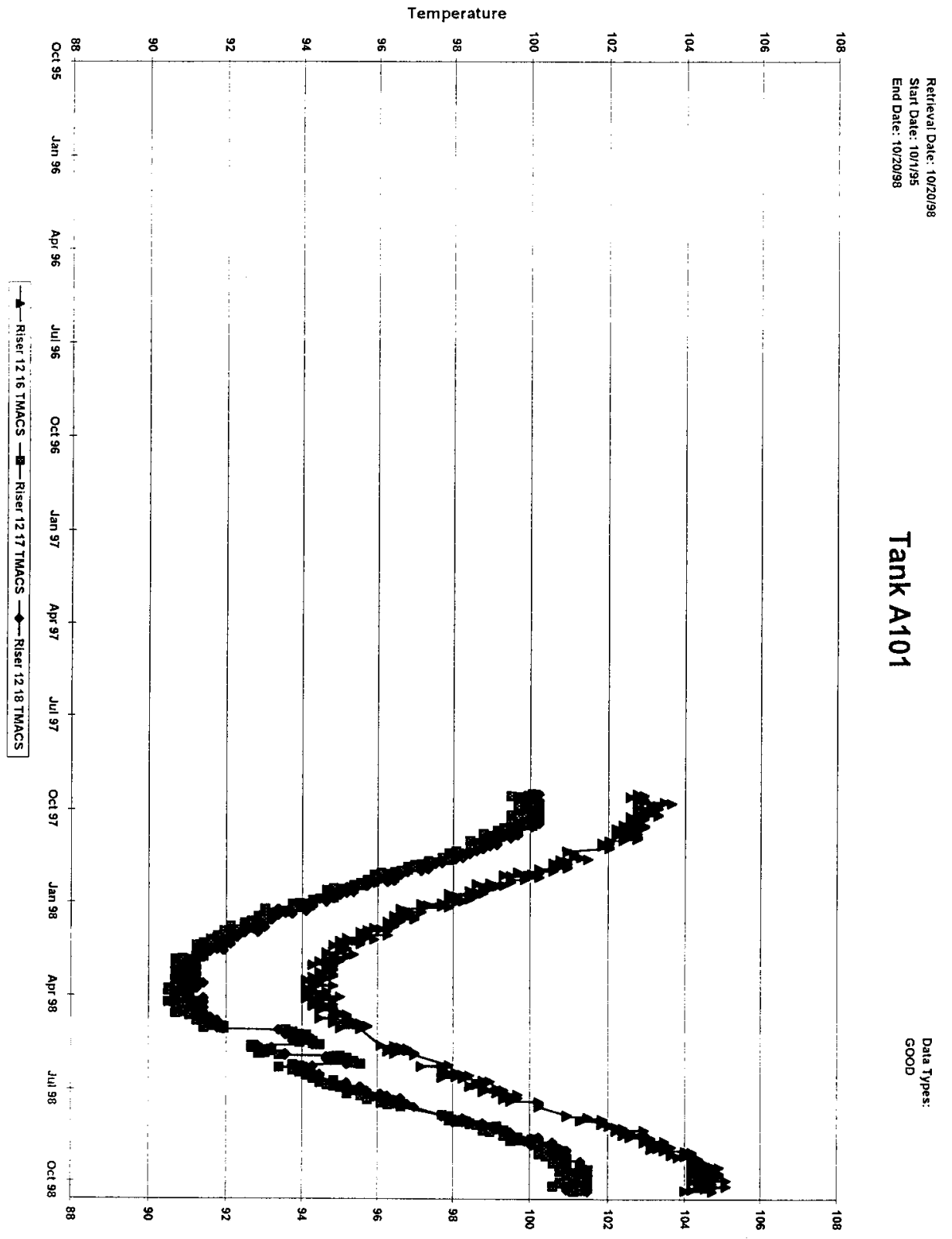




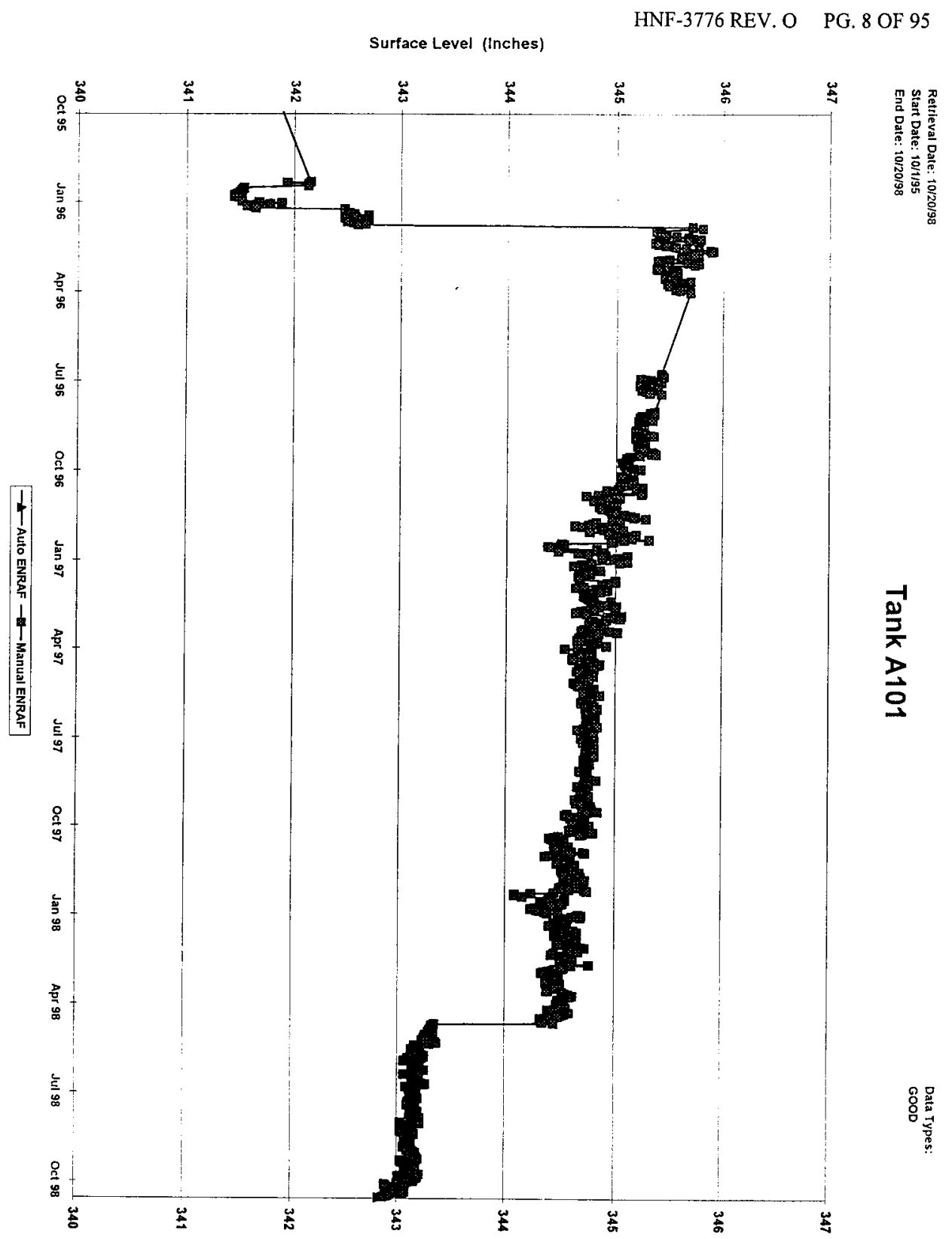


TMACS

Tagname

(null)

(null)

(null)

(null)

(nuil)

(nuli)

(null)

(null)

(null)

(null)

(null)

(null)

(null)

(null)

(null)

(null)

(null)

(null)

(null)

(null)

(null)

(null)

(null)

(null)

(nuli)

(nuli)

(null)

(nuil)

(nuli)

(nuli)

(null)

(nuli)

(null)

(null)

(null)

(null)

(null)

(null)

(null)

(nult)

inuli)

(null)

(null)

(nuli)

(null)

(null)
SACS

ID

A101-RISER-12-1

A101-RISER-12-10

A101-RISER-12-11

A101-RISER-12-12

A101-RISER-12-13

A101-RISER-12-14

A 101-RISER-12-15

A 101-RISER-12-16

A101-RISER-12-17

A 101-RISER-12-18

A101-RISER-12-2

A101-RISER-12-3

A 101-RISER-12-4

A101-RISER-12-5

A101-RISER-12-6

A101-RISER-12-7

A101-RISER-12-8

A101-RISER-12-9

A102-RISER-7-1

A102-RISER-7-10

A102-RISER-7-11

A102-RISER-7-12

A102-RISER-7-13

A 102-RISER-7- 14

A102-RISER-7-15

A102-RISER-7-16

A102-RISER-7-17

A102-RISER-7-18

A102-RISER-7-2

A102-RISER-7-3

A102-RISER-7-4

A102-RISER-7-5

A102-RISER-7-6

A102-RISER-7-7

A102-RISER-7-8

A 102-RISER-7-9

A.103-RISER-15-1

A103-RISER-15-10

A103-RISER-15-11

A103-RISER-15-12

A.103-RISER-15-13

A. 103-RISER-15-14

A103-RISER-15-15

A 103-RISER-15-15

A1 103-RISER-15-17

A.103-RISER-15-18
Height

Above

Bottom

3.96

219.96

243.96

267.96

291.96

315.96

339.96

363.96

411.96

459.96

27.96

51.96

75.96

99.96

123.96

147.96

171.96

195.96

4.00

220.00

244.00

268.00

292.00

316.00

340.00

364.00

412.00

460.00

28.00

52.00

76.00

100.00

124.00

148.00

172.00

196.00

3.96

219.96

243.96

267.96

291.96

315.96

339.96

363.96

411.96

459.96
Collection

System

HDAT

HDAT

HDAT

HDAT

HDAT

HDAT

HDAT

HDAT

HDAT

HDAT

HDAT

HDAT

HDAT

HDAT

HDAT

HDAT

HDAT

HDAT

HDAT

HDAT

HDAT

HDAT

HDAT

HDAT

HDAT

HDAT

HDAT

HDAT

HDAT

HDAT

HDAT

HDAT

HDAT

HDAT

HDAT

HDAT

HDAT

HDAT

HDAT

HDAT

HDAT

HDAT

HDAT

HDAT

HDAT

HDAT 
HNF-3776 REV. O PG. 10 OF 95

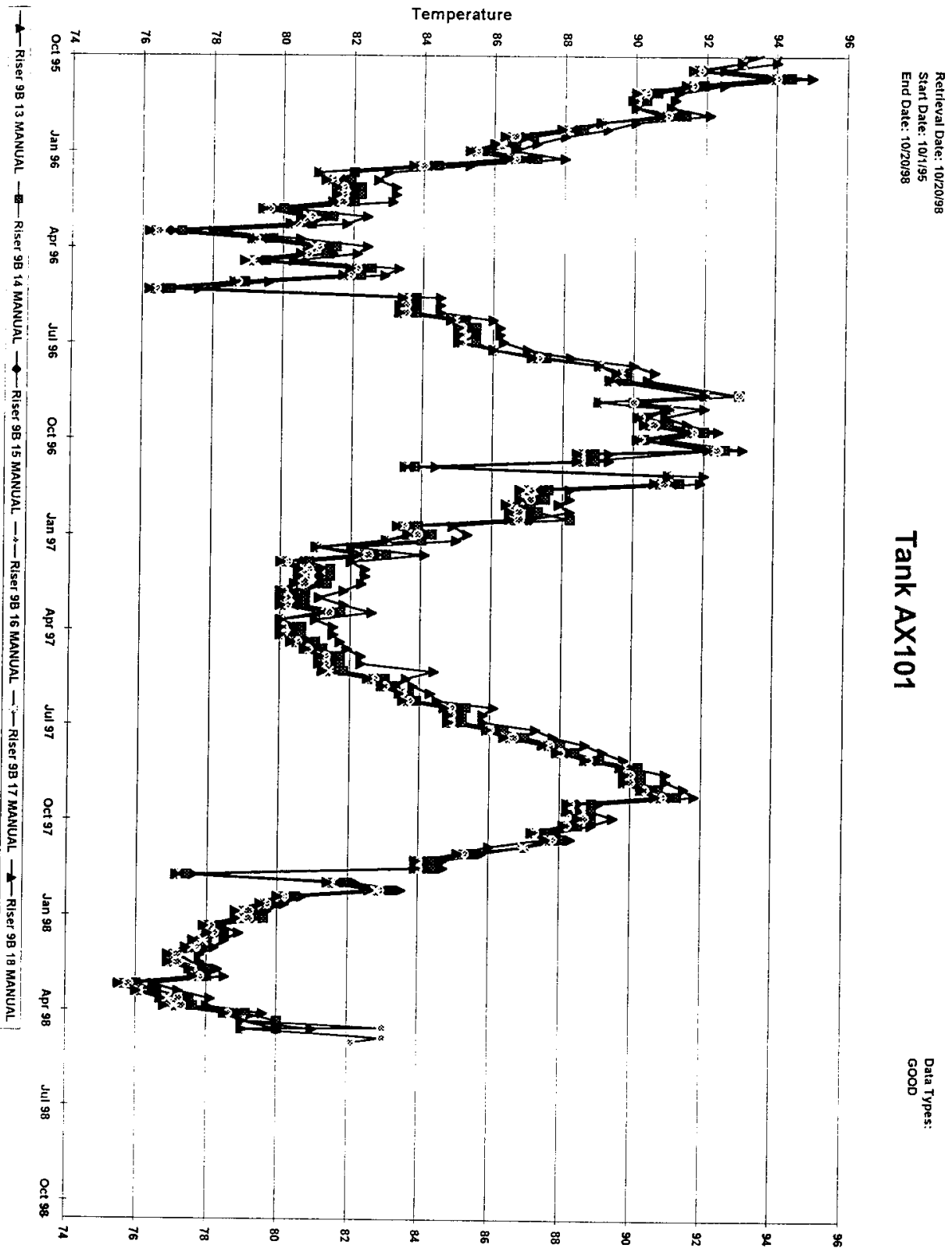


HNF-3776 REV. O PG. 11 OF 95

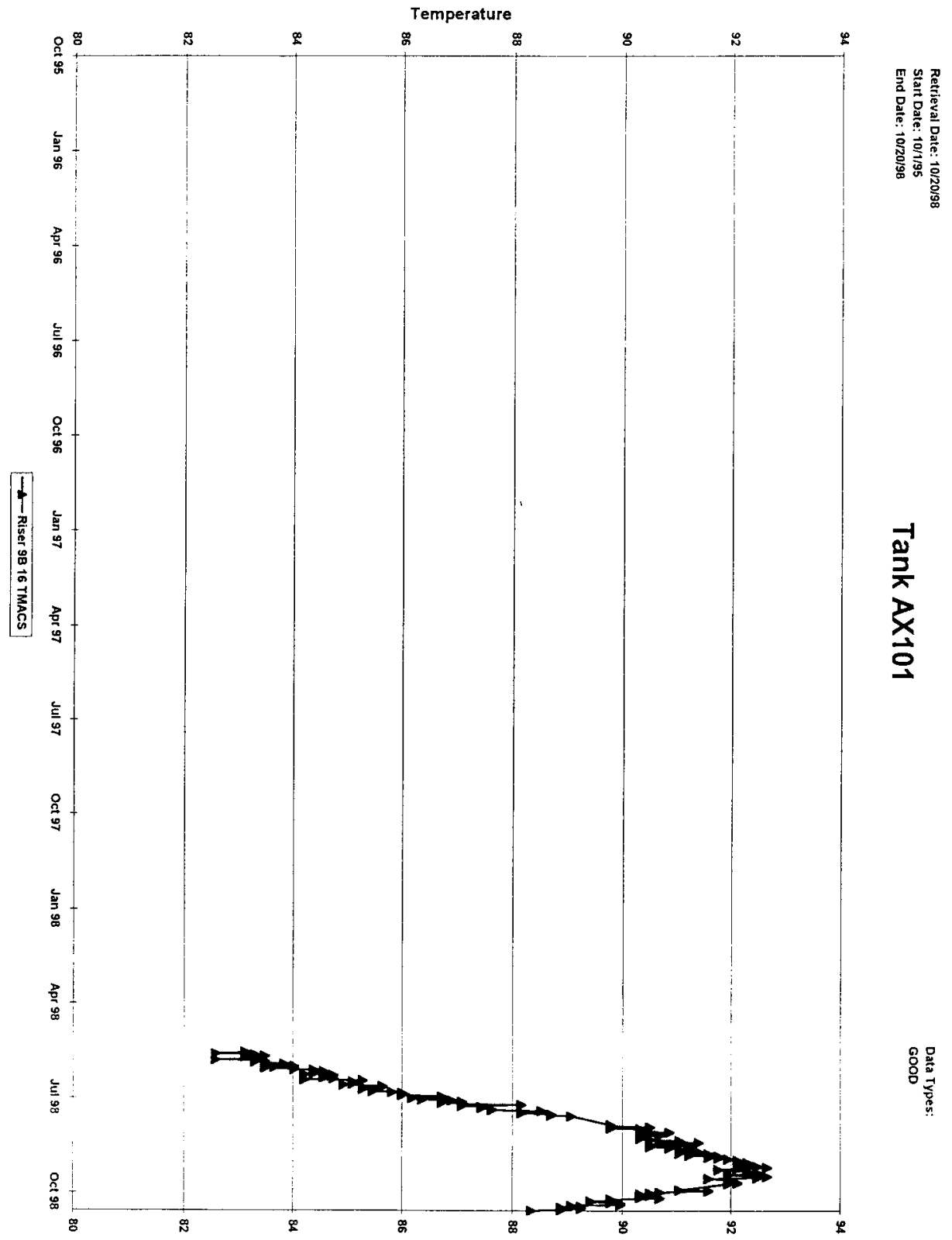




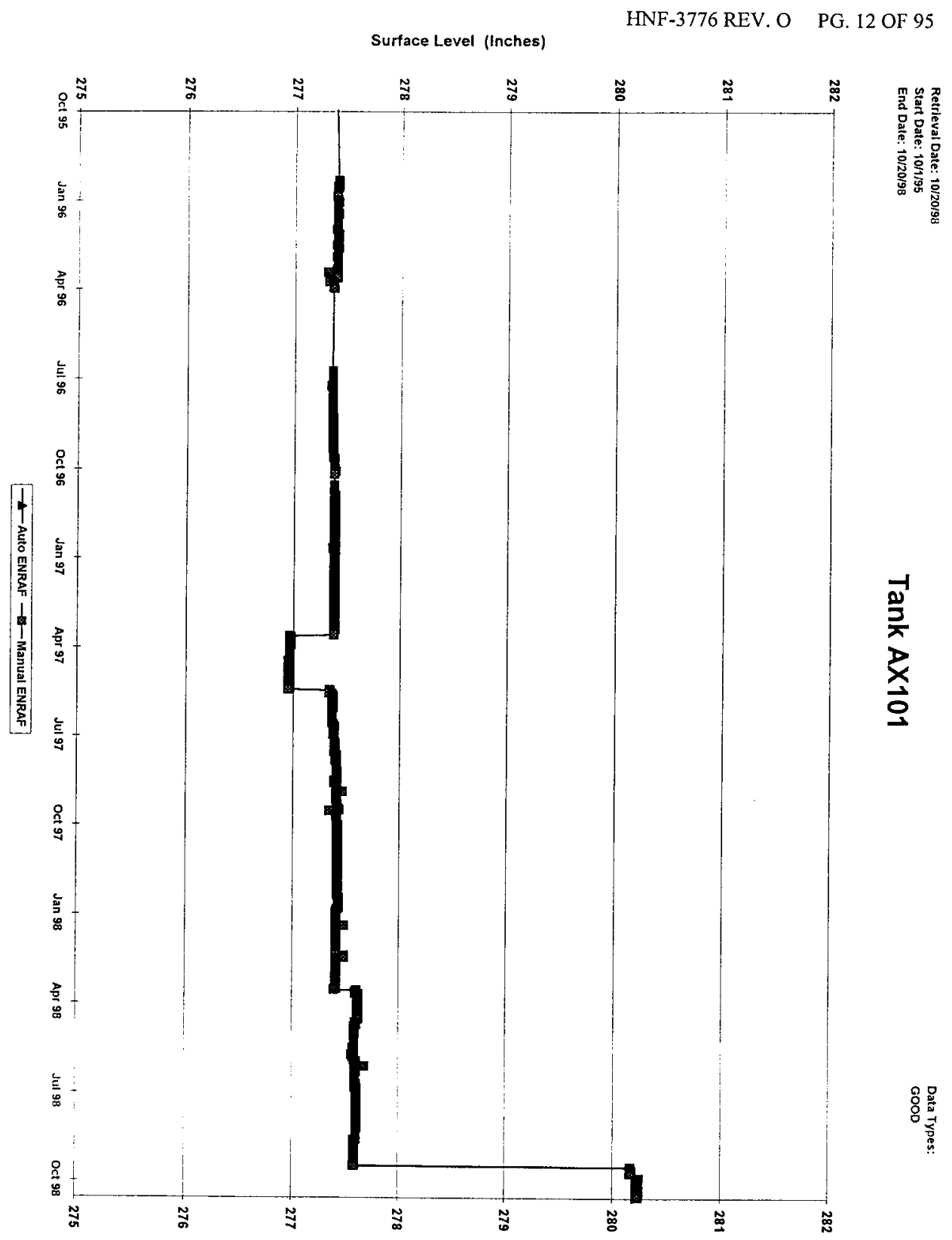




\begin{tabular}{|c|c|c|}
\hline (null) & AW-106-RISER-4A-9 & 195.96 \\
\hline (null) & AX101-RISER-9B-1 & 6.00 \\
\hline (nuili) & AX101-RISER-9B-10 & 222.00 \\
\hline (null) & AX101-RISER-9B-11 & 246.00 \\
\hline (null) & AX101-RISER-9B-12 & 270.00 \\
\hline (nuli) & AX101-RISER-9B-13 & 294.00 \\
\hline (null) & AX101-RISER-9B-14 & 318.00 \\
\hline (null) & AX101-RISER-9B-15 & 342.00 \\
\hline (null) & AX101-RISER-9B-16 & 366.00 \\
\hline (null) & AX101-RISER-9B-17 & 414.00 \\
\hline (null) & AX101-RISER-9B-18 & 462.00 \\
\hline (null) & AX101-RISER-9B-2 & 30.00 \\
\hline (null) & AX101-RISER-9B-3 & 54.00 \\
\hline (null) & AX101-RISER-9B-4 & 78.00 \\
\hline (null) & AX101-RISER-9B-5 & 102.00 \\
\hline (nuil) & AX101-RISER-9B-6 & 126.00 \\
\hline (null) & AX101-RISER-9B-7 & 150.00 \\
\hline (null) & AX101-RISER-9B-8 & 174.00 \\
\hline (null) & AX101-RISER-9B-9 & 198.00 \\
\hline (null) & AX102-RISER-9C-1 & 13.08 \\
\hline (null) & AX102-RISER-9C-10 & 229.08 \\
\hline (null) & AX102-RISER-9C-11 & 253.08 \\
\hline (null) & AX102-RISER-9C-12 & 277.08 \\
\hline (null) & AX102-RISER-9C-13 & 301.08 \\
\hline (nuil) & AX102-RISER-9C-14 & 325.08 \\
\hline (null) & AX102-RISER-9C-15 & 349.08 \\
\hline (null) & AX102-RISER-9C-16 & 373.08 \\
\hline (null) & AX102-RISER-9C-17 & 421.08 \\
\hline (null) & AX102-RISER-9C-18 & 469.08 \\
\hline (nuli) & AX102-RISER-9C-2 & 37.08 \\
\hline (null) & AX102-RISER-9C-3 & 61.08 \\
\hline (null) & AX102-RISER-9C-4 & 85.08 \\
\hline (null) & AX102-RISER-9C-5 & 109.08 \\
\hline (null) & AX102-RISER-9C-6 & 133.08 \\
\hline (null) & AX102-RISER-9C-7 & 157.08 \\
\hline (null) & AX102-RISER-9C-8 & 181.08 \\
\hline (null) & AX102-RISER-9C-9 & 205.08 \\
\hline (null) & AX103-R|SER-13C-1 & 2.04 \\
\hline (null) & AX103-RISER-13C-2 & 26.04 \\
\hline (nuill) & AX103-RISER-13C-3 & 50.04 \\
\hline (nuil) & AX103-RISER-13C-4 & 74.04 \\
\hline (null) & AX103-RISER-13C-5 & 98.04 \\
\hline (null) & AX103-RISER-13C-6 & 365.04 \\
\hline (nuli) & $\therefore \times 104-R I S E R-9 C-1$ & 12.84 \\
\hline (null) & $\dot{A} \times 104-R I S E R-9 C-10$ & 228.84 \\
\hline (null) & AX104-RISER-9C-11 & 252.84 \\
\hline (null) & $2 \times 104-R I S E R-9 C-12$ & 276.84 \\
\hline (null) & $A \times 104-R I S E R-9 C-13$ & 300.84 \\
\hline (nuli) & AX104-RISER-9C-14 & 324.84 \\
\hline (null) & AX104-RISER-9C-15 & 348.84 \\
\hline (null) & A $\times 104$-RISER-9C-16 & 372.84 \\
\hline
\end{tabular}


HNF-3776 REV. O PG. 14 OF 95

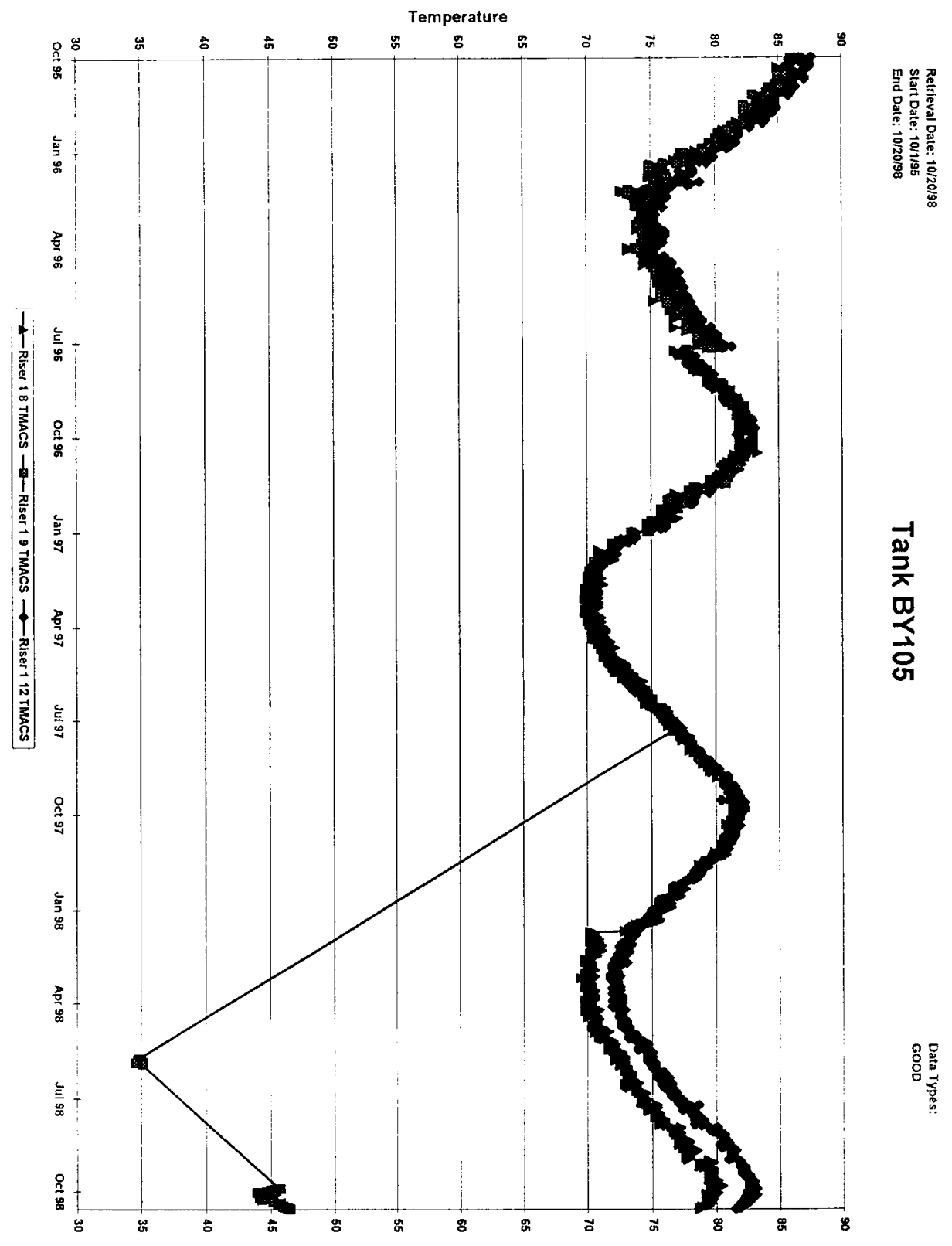


Surface Level (Inches)

HNF-3776 REV. O PG. 15 OF 95

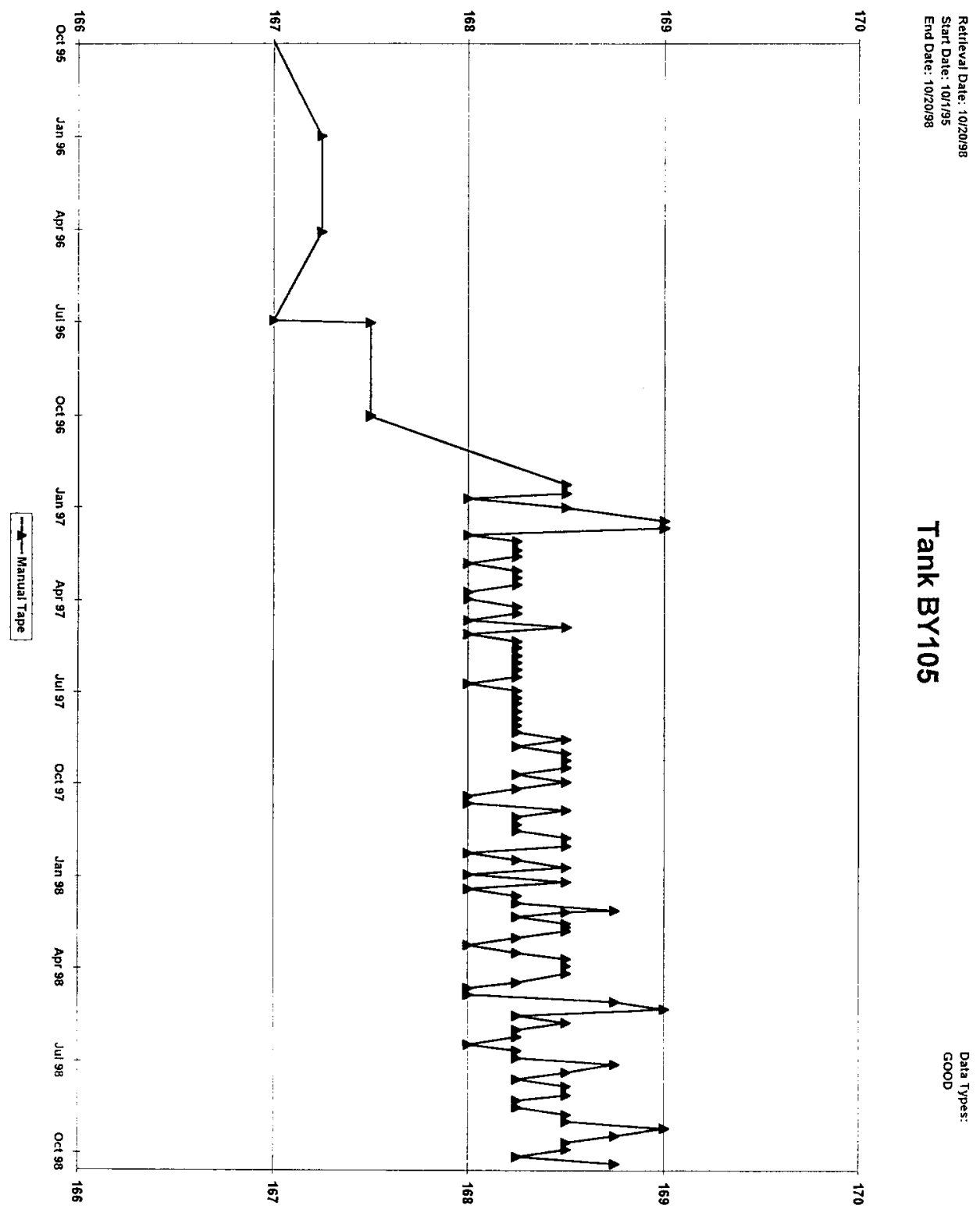


Sheet1

\begin{tabular}{|c|c|c|c|}
\hline TMACS & BY104-TI-R001-05 & BY104-RISER-1-5 & 102.00 \\
\hline TMACS & BY104-TI-R001-06 & BY104-RISER-1-6 & 290.00 \\
\hline TMACS & BY104-TI-R108-01 & BY104-RISER-10B-1 & 5.00 \\
\hline TMACS & BY104-TI-R10B-02 & BY104-RISER-10B-2 & 28.00 \\
\hline TMACS & BY104-TI-R10B-03 & BY104-RISER-10B-3 & 52.00 \\
\hline TMACS & BY104-TI-R108-04 & BY104-RISER-10B-4 & 88.00 \\
\hline TMACS & BY104-TI-R108-05 & BY104-RISER-10B-5 & 124.00 \\
\hline TMACS & BY104-TI-R10B-06 & BY104-RISER-10B-6 & 292.00 \\
\hline TMACS & BY105-TI-R001-01 & BY 105-RISER-1-1 & 4.00 \\
\hline TMACS & BY105-TI-R001-02 & BY105-RISER-1-2 & 28.00 \\
\hline TMACS & BY105-TI-R001-03 & BY105-RISER-1-3 & 52.00 \\
\hline TMACS & BY105-TI-R001-04 & BY105-RISER-1-4 & 76.00 \\
\hline TMACS & BY105-TI-R001-05 & BY 105-RISER-1-5 & 100.00 \\
\hline TMACS & BY105-TI-R001-06 & BY105-RISER-1-6 & 124.00 \\
\hline TMACS & BY105-TI-R001-07 & BY105-RISER-1-7 & 148.00 \\
\hline TMACS & BY105-TI-R001-08 & BY105-RISER-1-8 & 172.00 \\
\hline TMACS & BY105-TI-R001-09 & BY105-RISER-1-9 & 196.00 \\
\hline TMACS & BY105-TI-R001-10 & BY105-RISER-1-10 & 220.00 \\
\hline TMACS & BY105-TI-R001-11 & BY105-RISER-1-11 & 244.00 \\
\hline TMACS & BY105-TI-R001-12 & BY105-RISER-1-12 & 268.00 \\
\hline TMACS & BY105-TI-R10C01 & BY105-RISER-10C-1 & 8.00 \\
\hline TMACS & BY105-TI-R10CO2 & BY105-RISER-10C-2 & 32.00 \\
\hline TMACS & BY105-TI-R10C03 & BY105-RISER-10C-3 & 56.00 \\
\hline TMACS & BY105-TI-R10CO4 & BY105-RISER-10C-4 & 80.00 \\
\hline TMACS & BY105-TI-R10C05 & BY105-RISER-10C-5 & 104.00 \\
\hline TMACS & BY105-TI-R10C06 & BY105-RISER-10C-6 & 296.00 \\
\hline TMACS & BY106-TI-R001-01 & BY106-RISER-1-1 & 8.00 \\
\hline TMACS & BY106-TI-R001-02 & BY $106-R I S E R-1-2$ & 32.00 \\
\hline TMACS & BY106-TI-R001-03 & BY106-RISER-1-3 & 56.00 \\
\hline TMACS & BY106-TI-R001-04 & BY 106-RISER-1-4 & 80.00 \\
\hline TMACS & BY106-TI-R001-05 & BY106-RISER-1-5 & 104.00 \\
\hline TMACS & BY106-TI-R001-06 & BY106-RISER-1-6 & 296.00 \\
\hline TMACS & BY107-TI-R001-01 & BY107-RISER-1-1 & 6.00 \\
\hline TMACS & BY107-TI-R001-02 & BY107-RISER-1-2 & 30.00 \\
\hline TMACS & BY107-TI-R001-03 & BY107-RISER-1-3 & 54.00 \\
\hline TMACS & BY107-TI-R001-04 & BY 107-RISER-1-4 & 78.00 \\
\hline TMACS & BY107-TI-R001-05 & BY107-RISER-1-5 & 102.00 \\
\hline TMACS & BY107-TI-R001-06 & BY107-RISER-1-6 & 126.00 \\
\hline TMACS & BY107-TI-R001-07 & BY107-RISER+1-7 & 150.00 \\
\hline TMACS & BY107-TI-R001-08 & BY107-RISER-1-8 & 174.00 \\
\hline TMACS & BY107-TI-R001-09 & BY107-RISER-1-9 & 198.00 \\
\hline TMACS & BY107-TI-R001-10 & BY107-RISER-1-10 & 222.00 \\
\hline TMACS & BY107-TI-R001-11 & BY107-RISER-1-11 & 246.00 \\
\hline TMACS & BY 107-TI-R001-12 & BY 107-RISER-1-12 & 270.00 \\
\hline TMACS & BY107-TI-R001-13 & BY107-RISER-1-13 & 318.00 \\
\hline TMACS & BY107-TI-R001-14 & BY107-RISER-1-14 & 366.00 \\
\hline TMACS & BY107-TI-R005-01 & BY107-RISER-5-1 & 4.00 \\
\hline TMACS & BY107-TI-R005-02 & EY107-RISER-5-2 & 9.00 \\
\hline TMACS & BY107-TI-R005-03 & BY107-RISER-5-3 & 15.00 \\
\hline TMACS & BY107-TI-R005-04 & BY107-RISER-5-4 & 27.00 \\
\hline TMACS & BY107-TI-R005-05 & BY107-RISER-5-5 & 45.00 \\
\hline
\end{tabular}


HNF-3776 REV. O PG. 17 OF 95

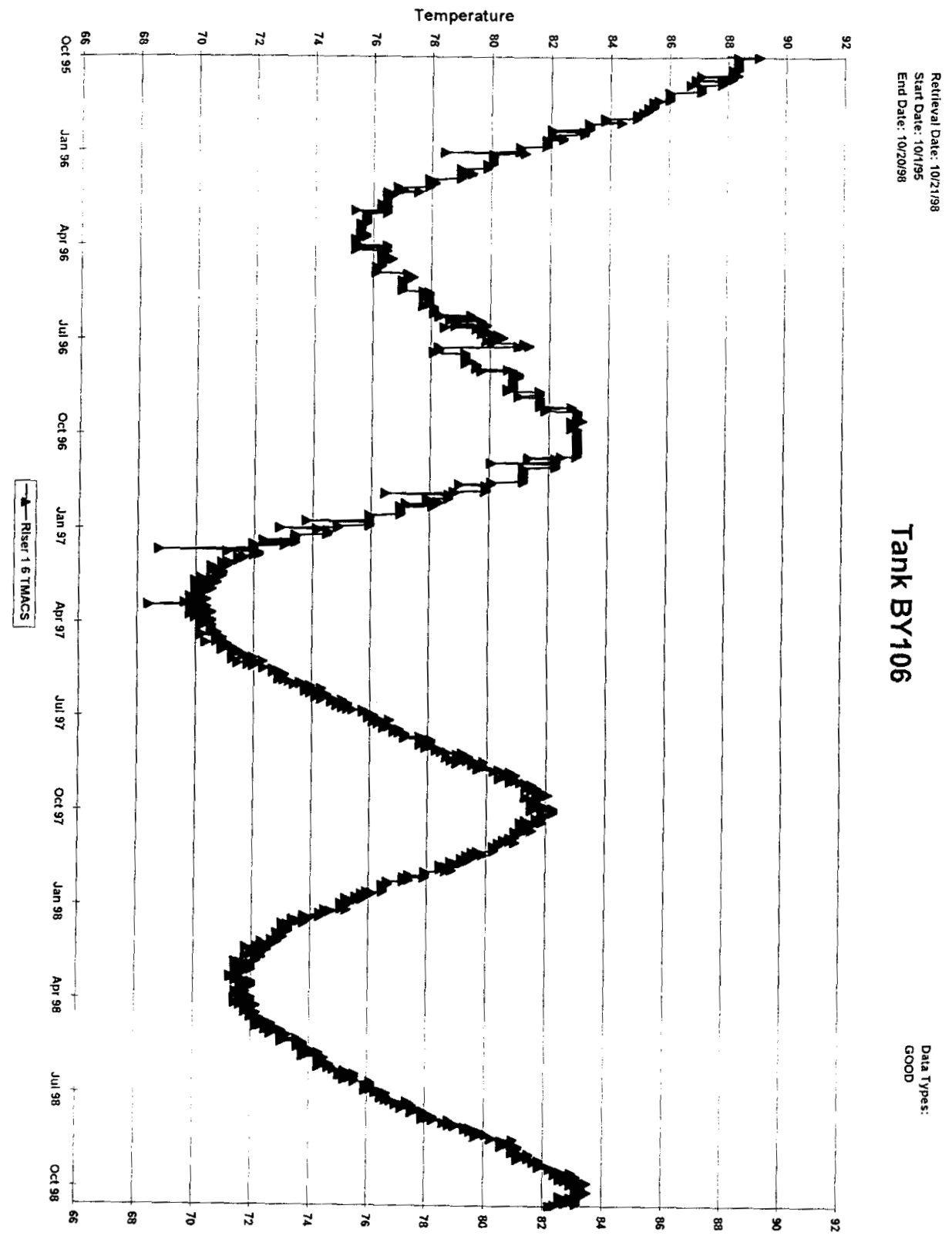


HNF-3776 REV. O PG. 18 OF 95

Surface Level (Inches)

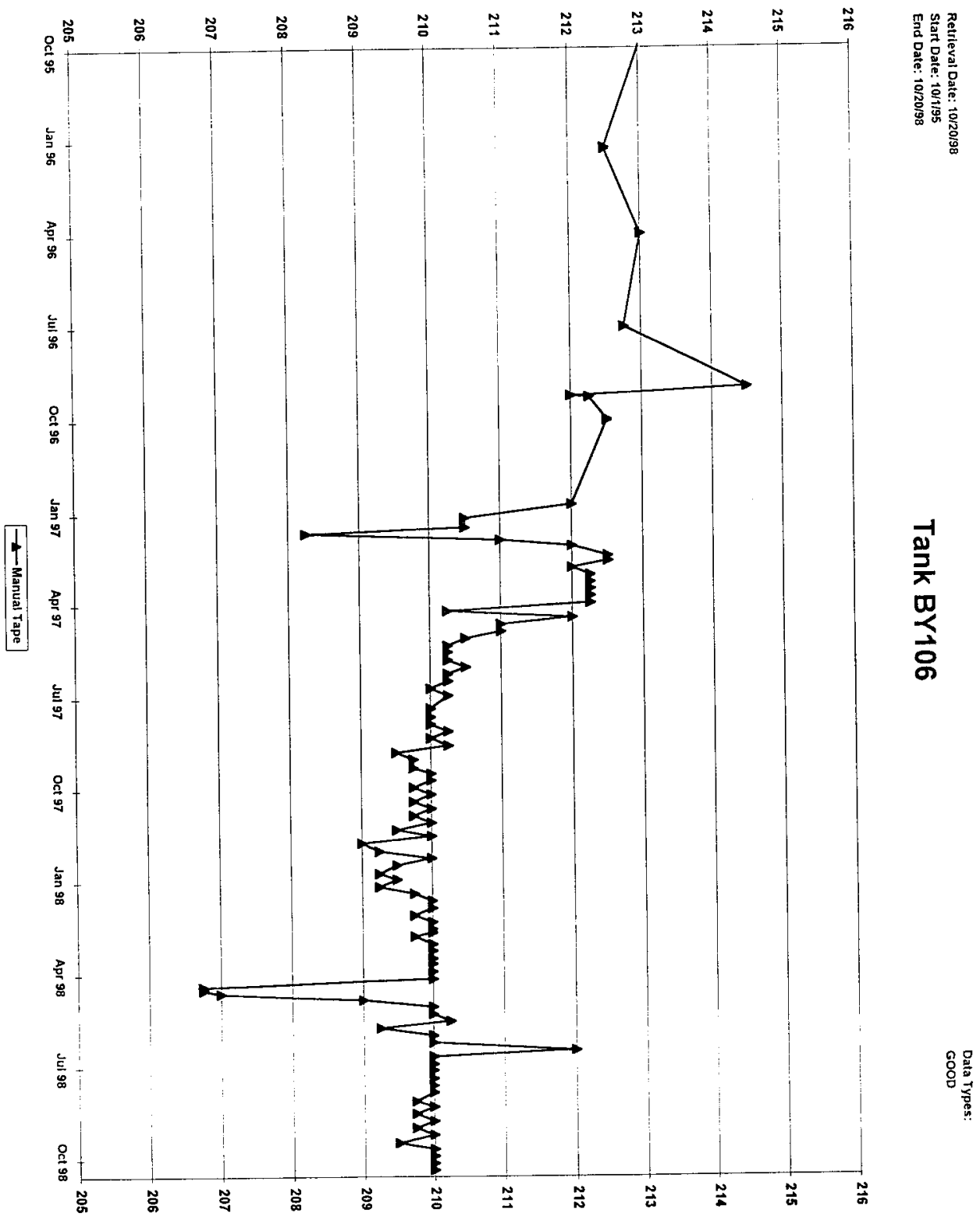


TMACS TMACS

TMACS

TMACS

TMACS

TMACS

TMACS

TMACS

TMACS

TMACS

TMACS

TMACS

TMACS

TMACS

TMACS

TMACS

TMACS

TMACS

TMACS

TMACS

TMACS

TMACS

TMACS

TMACS

TMACS

TMACS

TMACS

TMACS
BY105-TI-R10C04 BY105-TI-R10C05 BY105-TI-R10C06 BY106-TI-R001-01 BY106-TI-R001-02 BY106-TI-R001-03 BY106-TI-R001-04 BY106-TI-R001-05 BY106-TI-R001-06 BY107-TI-R001-01 BY107-TI-R001-02 BY107-TI-R001-03 BY107-TI-R001-04 BY107-TI-R001-05 BY107-TI-R001-06 BY107-TI-R001-07 BY107-TI-R001-08 BY107-TI-R001-09 BY107-TI-R001-10 BY107-TI-R001-11 BY107-TI-R001-12 BY107-TI-R001-13 BY107-TI-R001-14 BY107-TI-R005-01 BY107-TI-R005-02 BY107-TI-R005-03 BY107-TI-R005-04 BY107-TI-R005-05
BY105-RISER-10C-4 80.00 BY105-RISER-10C-5 104.00 BY105-RISER-10C-6 296.00 BY106-RISER-1-1 8.00 BY106-RISER-1-2 $\quad 32.00$ BY106-RISER-1-3 56.00 BY106-RISER-1-4 80.00 BY106-RISER-1-5 104.00 BY106-RISER-1-6 296.00 BY107-RISER-1-1 6.00 BY107-RISER-1-2 30.00 BY107-RISER-1-3 54.00 BY107-RISER-1-4 78.00 BY107-RISER-1-5 102.00 BY107-RISER-1-6 126.00 BY107-RISER-1-7 150.00 BY107-RISER-1-8 174.00 BY107-RISER-1-9 198.00 BY107-RISER-1-10 222.00 BY107-RISER-1-11 246.00 BY107-RISER-1-12 270.00 BY107-RISER-1-13 318.00 BY107-RISER-1-14 366.00 BY107-RISER-5-1 4.00 BY107-RISER-5-2 9.00 BY107-RISER-5-3 15.00 BY107-RISER-5-4 27.00 BY107-RISER-5-5 45.00 
Surface Level (Inches)

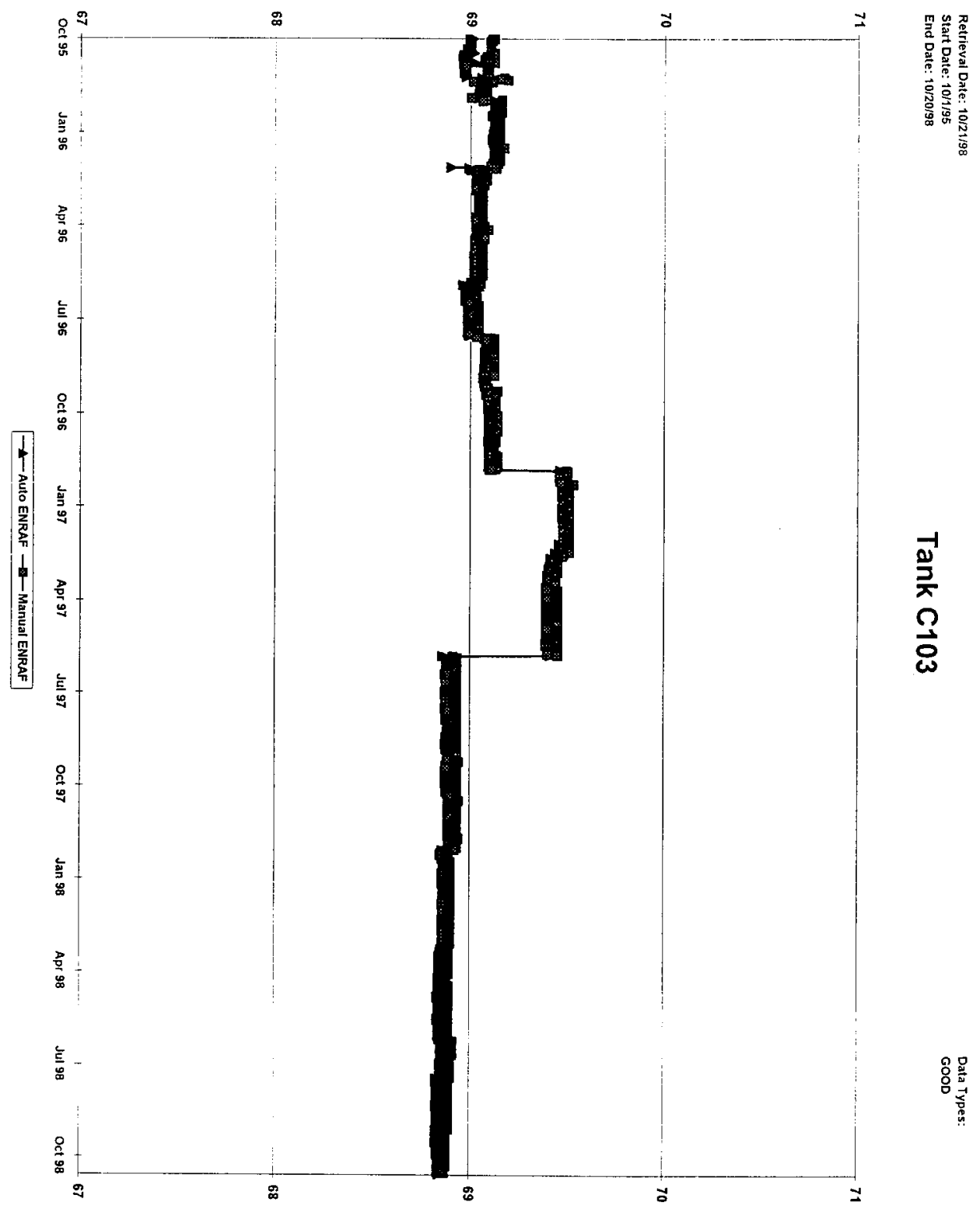


HNF-3776 REV. O PG. 21 OF 95

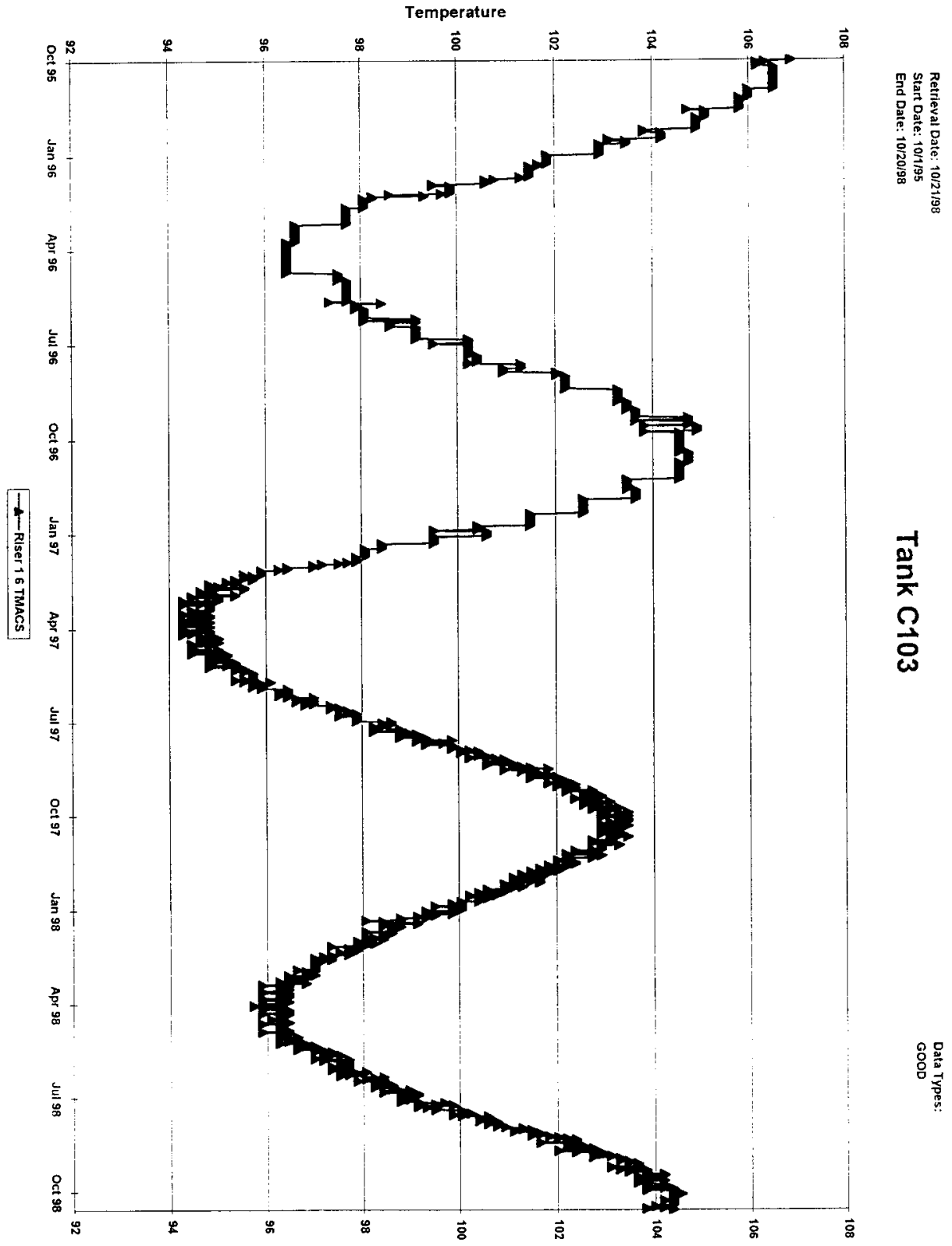




\begin{tabular}{|c|c|c|}
\hline (null) & C101-RISER-2-11 & 303.96 \\
\hline (null) & C101-RISER-2-2 & 39.96 \\
\hline (null) & C101-RISER-2-3 & 63.96 \\
\hline (null) & C101-RISER-2-4 & 87.96 \\
\hline (null) & C101-RISER-2-5 & 111.96 \\
\hline (null) & C101-RISER-2-6 & 135.96 \\
\hline (null) & C101-RISER-2-7 & 159.96 \\
\hline (null) & C101-RISER-2-8 & 183.96 \\
\hline (null) & C101-RISER-2-9 & 207.96 \\
\hline (nuil) & C102-RISER-7-1 & 15.96 \\
\hline (nul!) & C102-RISER-7-10 & 255.96 \\
\hline (null) & C102-RISER-7-11 & 303.96 \\
\hline (nuli) & C102-RISER-7-2 & 39.96 \\
\hline (null) & C102-RISER-7-3 & 63.96 \\
\hline (null) & C102-RISER-7-4 & 87.96 \\
\hline (nuli) & C102-RISER-7-5 & 111.96 \\
\hline (null) & C102-RISER-7-6 & 135.96 \\
\hline (null) & C102-RISER-7-7 & 159.96 \\
\hline (null) & C102-RISER-7-8 & 183.96 \\
\hline (null) & C102-RISER-7-9 & 207.96 \\
\hline (null) & C103-RISER-1-1 & 14.52 \\
\hline (null) & C103-RISER-1-2 & 38.04 \\
\hline (null) & C103-RISER-1-3 & 62.04 \\
\hline (null) & C103-RISER-1-4 & 86.04 \\
\hline (null) & C103-RISER-1-5 & 110.04 \\
\hline (null) & C103-RISER-1-6 & 192.00 \\
\hline (null) & C104-RISER-7-1 & 15.96 \\
\hline (null) & C104-RISER-7-10 & 231.96 \\
\hline (null) & C104-RISER-7-11 & 255.96 \\
\hline (null) & C104-RISER-7-12 & 279.96 \\
\hline (null) & C104-RISER-7-2 & 39.96 \\
\hline (null) & C104-RISER-7-3 & 63.96 \\
\hline (nuli) & C104-RISER-7-4 & 87.96 \\
\hline (null) & C104-RISER-7-5 & 111.96 \\
\hline (null) & C104-RISER-7-6 & 135.96 \\
\hline (null) & C104-RISER-7-7 & 159.96 \\
\hline (null) & C104-RISER-7-8 & 183.96 \\
\hline (null) & C104-RISER-7-9 & 207.96 \\
\hline (null) & C105-RISER-1-1 & 14.40 \\
\hline (null) & C105-RISER-1-2 & 37.92 \\
\hline (nuli) & C105-RISER-1-3 & 61.92 \\
\hline (null) & C105-RISER-1-4 & 85.92 \\
\hline (null) & C105-RISER-1-5 & 109.92 \\
\hline (null) & C105-RISER-1-6 & 191.88 \\
\hline (null) & C:06-RISER-14-1 & 15.96 \\
\hline (null) & C106-RISER-14-10 & 231.96 \\
\hline (null) & C106-RISER- $14-11$ & 255.96 \\
\hline (null) & 0106-RISER-14-12 & 279.96 \\
\hline (nuil) & C106-RISER-14-2 & 39.96 \\
\hline dii) & C106-RISER-14-3 & 63.96 \\
\hline ull) & C106-RISER-14-4 & 87.96 \\
\hline
\end{tabular}




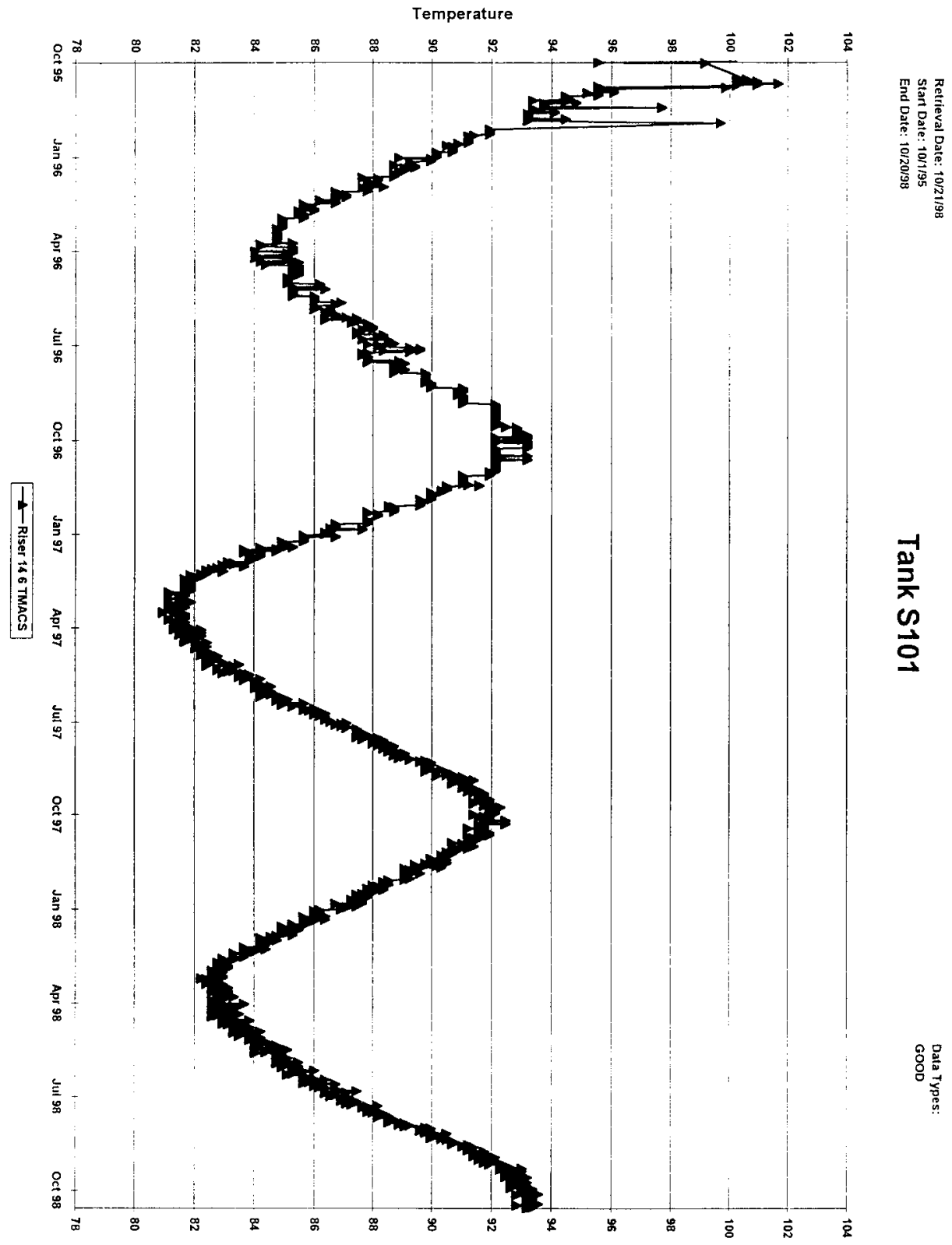


HNF-3776 REV. O PG. 24 OF 95

Surface Level (Inches)

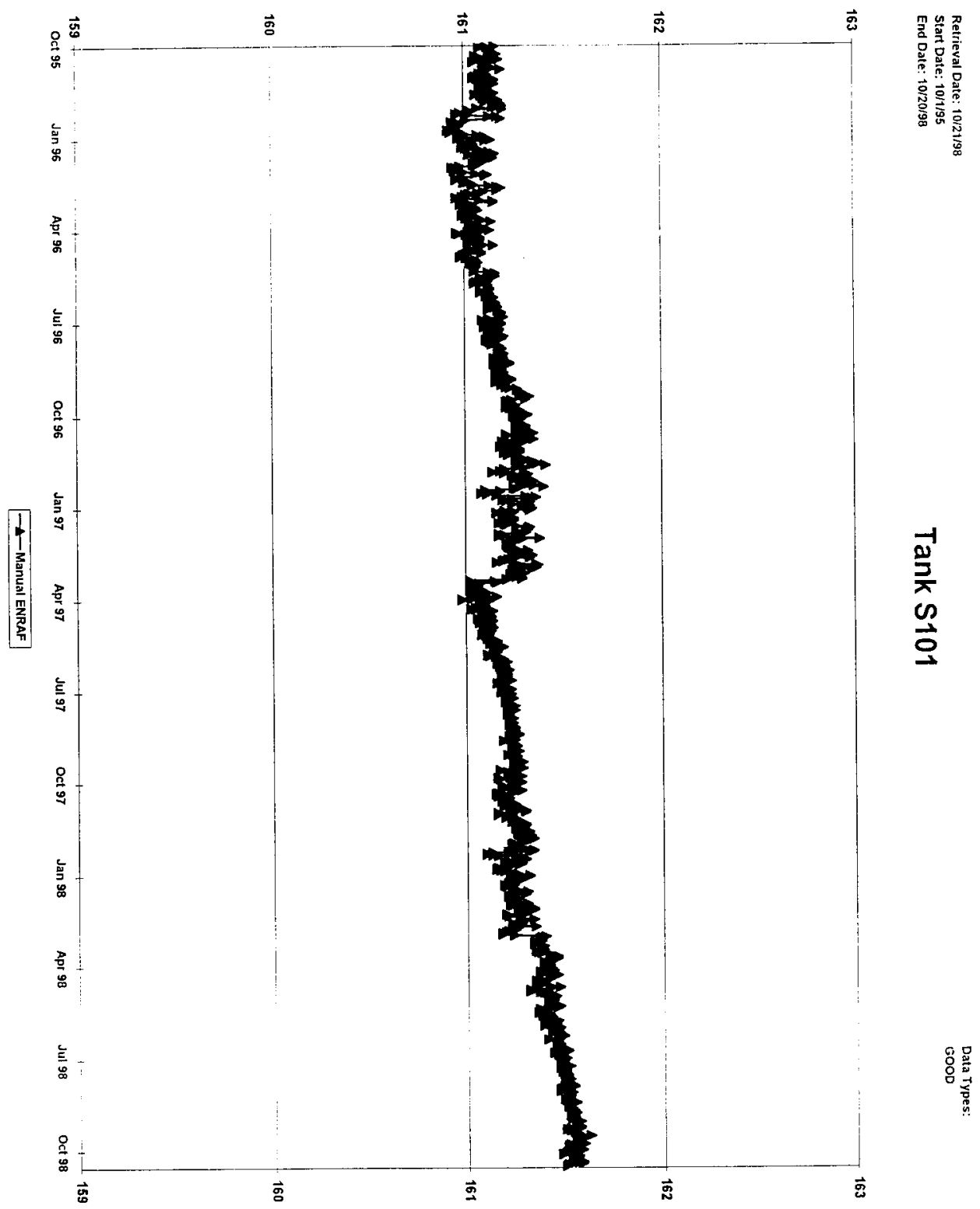


Sheet1

\begin{tabular}{|c|c|c|c|}
\hline $\begin{array}{l}\text { Collection } \\
\text { System }\end{array}$ & $\begin{array}{l}\text { TMACS } \\
\text { Tagname }\end{array}$ & $\begin{array}{l}\text { SACS } \\
\text { ID }\end{array}$ & $\begin{array}{l}\text { Height } \\
\text { Above } \\
\text { Bottom }\end{array}$ \\
\hline HDAT & (null) & S101-RISER-14-1 & 5.76 \\
\hline HDAT & (null) & S101-RISER-14-2 & 29.28 \\
\hline HDAT & (nuli) & S101-RISER-14-3 & 53.28 \\
\hline HDAT & (null) & S101-RISER-14-4 & 77.28 \\
\hline HDAT & (null) & S101-RISER-14-5 & 101.28 \\
\hline HDAT & (null) & S101-RISER-14-6 & 293.28 \\
\hline HDAT & (null) & S102-RISER-3-1 & 5.76 \\
\hline HDAT & (nulli) & S102-RISER-3-2 & 29.28 \\
\hline HDAT & (null) & S102-RISER-3-3 & 53.28 \\
\hline HDAT & (null) & S102-RISER-3-4 & 77.28 \\
\hline HDAT & (null) & S102-RISER-3-5 & 101.28 \\
\hline HDAT & (null) & S102-RISER-3-6 & 293.28 \\
\hline HDAT & (null) & S103-RISER-4-1 & 5.16 \\
\hline HDAT & (null) & S103-RISER-4-2 & 28.68 \\
\hline HDAT & (null) & S103-RISER-4-3 & 52.68 \\
\hline HDAT & (nulf) & S103-RISER-4-4 & 76.68 \\
\hline HDAT & (nuli) & S103-RISER-4-5 & 100.68 \\
\hline HDAT & (null) & S103-RISER-4-6 & 292.68 \\
\hline HDAT & (nuil) & S104-RISER-4-1 & 6.00 \\
\hline HDAT & (null) & S104-RISER-4-10 & 222.00 \\
\hline HDAT & (null) & S104-RISER-4-11 & 246.00 \\
\hline HDAT & (null) & S104-RISER-4-12 & 270.00 \\
\hline HDAT & (null) & S104-RISER-4-13 & 318.00 \\
\hline HDAT & (null) & S104-RISER-4-14 & 366.00 \\
\hline HDAT & (null) & S104-RISER-4-2 & 30.00 \\
\hline HDAT & (null) & S104-RISER-4-3 & 54.00 \\
\hline HDAT & (null) & S104-RISER-4-4 & 78.00 \\
\hline HDAT & (null) & S104-RISER-4-5 & 102.00 \\
\hline HDAT & (null) & S104-RISER-4-6 & 126.00 \\
\hline HDAT & (nuil) & S104-RISER-4-7 & 150.00 \\
\hline HDAT & (nuil) & S104-RISER-4-8 & 174.00 \\
\hline HDAT & (null) & S104-RISER-4-9 & 198.00 \\
\hline HDAT & (nuil) & S105-RISER-4-1 & 6.72 \\
\hline HDAT & (null) & S105-RISER-4-10 & 222.72 \\
\hline HDAT & (nuli) & S105-RISER-4-11 & 246.72 \\
\hline HDAT & (null) & S105-RISER-4-12 & 270.72 \\
\hline HDAT & (nutl) & S105-RISER-4-13 & 318.72 \\
\hline HDAT & (null) & S105-RISER-4-14 & 366.72 \\
\hline HDAT & (null) & S105-RISER-4-2 & 30.72 \\
\hline HDAT & (nuil) & S105-RISER-4-3 & 54.72 \\
\hline HDA' & (nullj) & S:05-RISER-4-4 & 78.72 \\
\hline HDAT & (null) & S105-RISER-4-5 & 102.72 \\
\hline HDA: & (nuil) & 3:05-RISER-4.6 & 126.72 \\
\hline HDAT & (null) & S:05-RISER-4-7 & 150.72 \\
\hline HDAT & (null) & S:05-RISER-4-3 & 174.72 \\
\hline HDAT & (null) & S105-RISER-4-9 & 198.72 \\
\hline
\end{tabular}




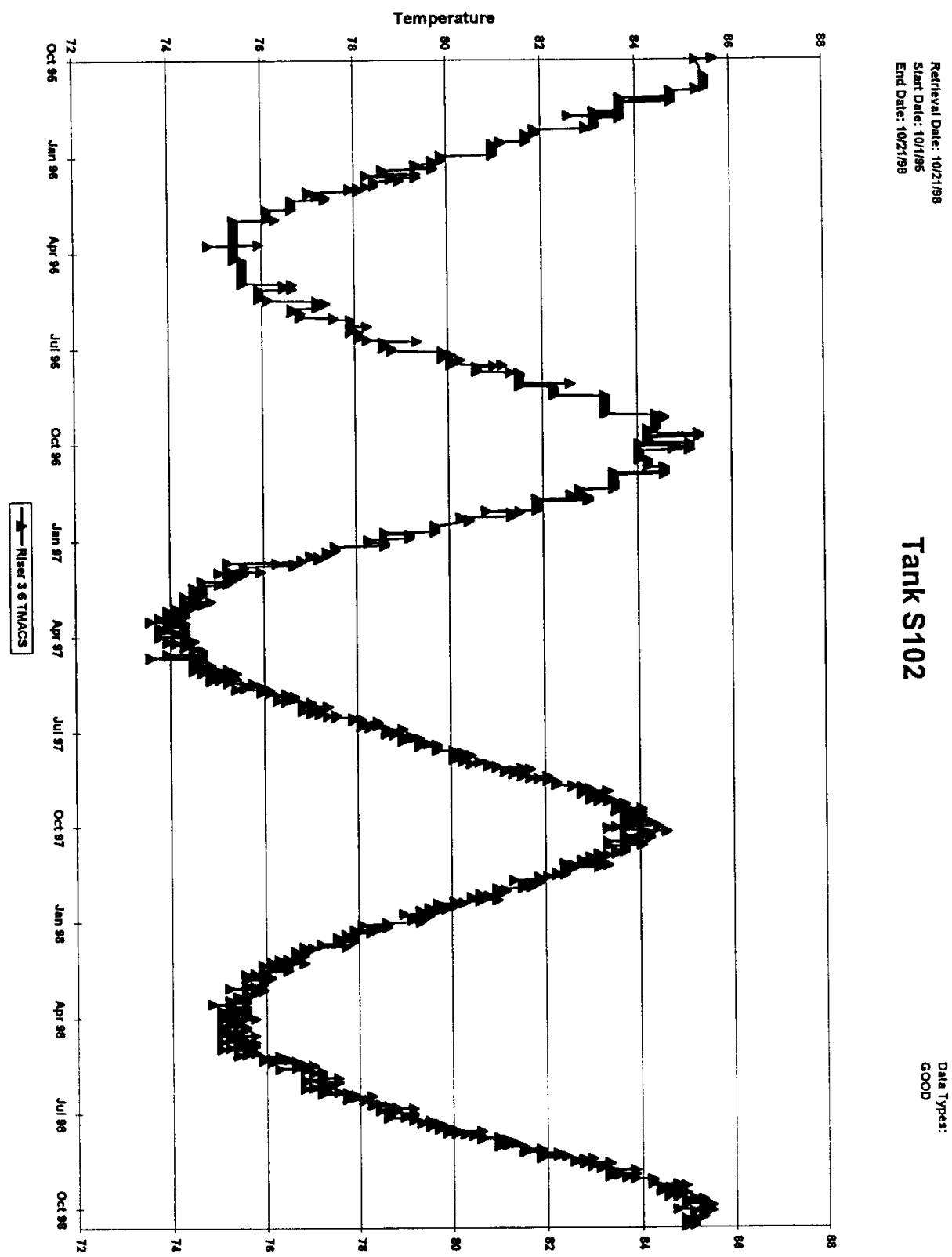


HNF-3776 REV. O PG. 27 OF 95

Surface Level (Inches)

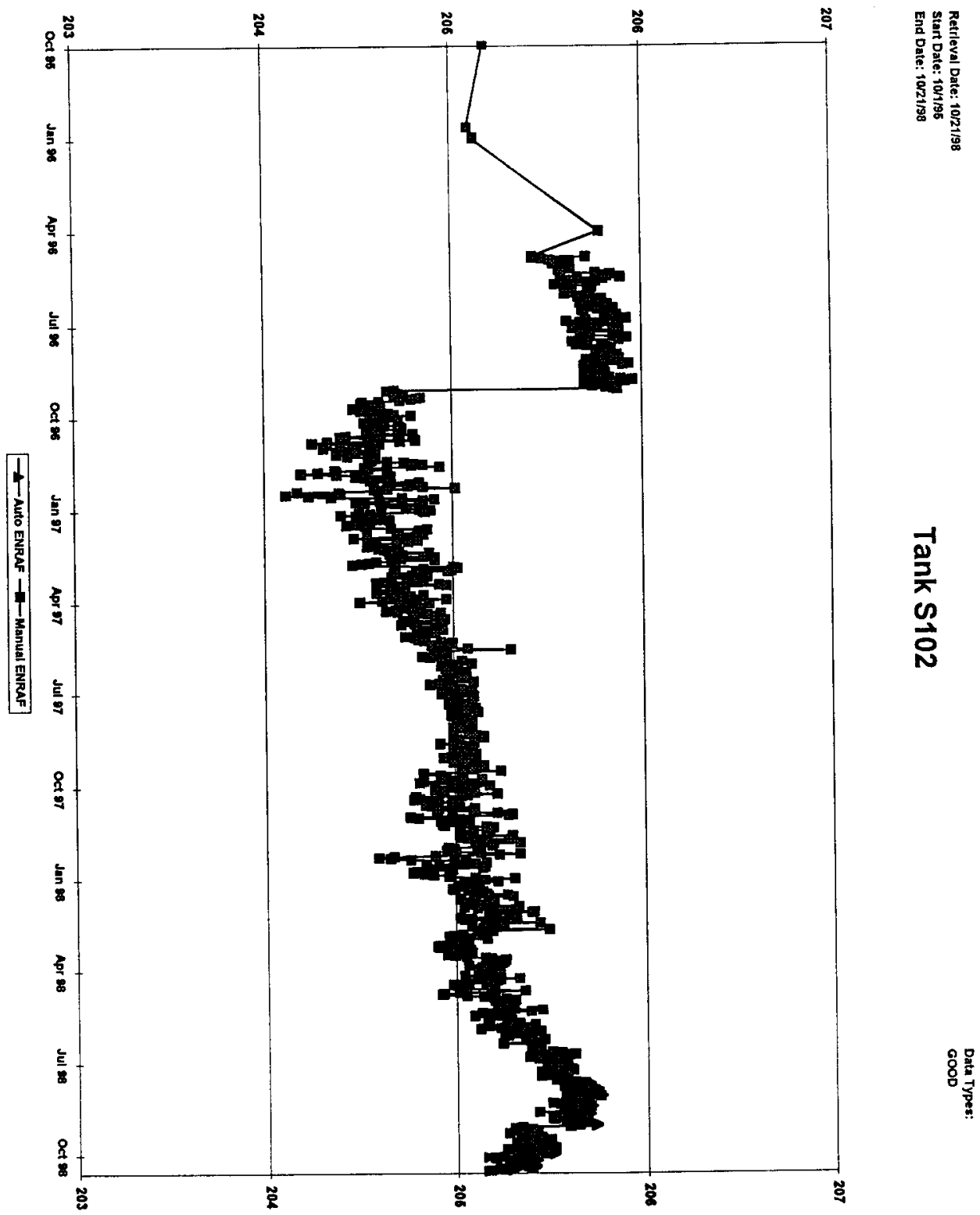


Sheet1

\begin{tabular}{|c|c|c|c|}
\hline $\begin{array}{l}\text { Collection } \\
\text { System }\end{array}$ & $\begin{array}{l}\text { TMACS } \\
\text { Tagname }\end{array}$ & $\begin{array}{c}\text { SACS } \\
\text { ID }\end{array}$ & $\begin{array}{l}\text { Height } \\
\text { Above } \\
\text { Bottom }\end{array}$ \\
\hline HDAT & (null) & S101-RISER-14-1 & 5.76 \\
\hline HDAT & (null) & S101-RISER-14-2 & 29.28 \\
\hline HDAT & (null) & S101-RISER-14-3 & 53.28 \\
\hline HDAT & (nuli) & S101-RISER-14-4 & 77.28 \\
\hline HDAT & (null) & S101-RISER-14-5 & 101.28 \\
\hline HDAT & (null) & S101-RISER-14-6 & 293.28 \\
\hline HDAT & (null) & S102-RISER-3-1 & 5.76 \\
\hline HDAT & (null) & S102-RISER-3-2 & 29.28 \\
\hline HDAT & (null) & S102-RISER-3-3 & 53.28 \\
\hline HDAT & (null) & S102-RISER-3-4 & 77.28 \\
\hline HDAT & (null) & S102-RISER-3-5 & 101.28 \\
\hline HDAT & (null) & S102-RISER-3-6 & 293.28 \\
\hline HDAT & (null) & S103-RISER-4-1 & 5.16 \\
\hline HDAT & (nuli) & S103-RISER-4-2 & 28.68 \\
\hline HDAT & (nuli) & S103-RISER-4-3 & 52.68 \\
\hline HDAT & (null) & S103-RISER-4-4 & 76.68 \\
\hline HDAT & (null) & S103-RISER-4-5 & 100.68 \\
\hline HDAT & (null) & S103-RISER-4-6 & 292.68 \\
\hline HDAT & (nutl) & S104-RISER-4-1 & 6.00 \\
\hline HDAT & (null) & S104-RISER-4-10 & 222.00 \\
\hline HDAT & (null) & S104-RISER-4-11 & 246.00 \\
\hline HDAT & (null) & S104-RISER-4-12 & 270.00 \\
\hline HDAT & (null) & S104-RISER-4-13 & 318.00 \\
\hline HDAT & (null) & S104-RISER-4-14 & 366.00 \\
\hline HDAT & (nuil) & S104-RISER-4-2 & 30.00 \\
\hline HDAT & (null) & S104-RISER-4-3 & 54.00 \\
\hline HDAT & (null) & S104-RISER-4-4 & 78.00 \\
\hline HDAT & (null) & S104-RISER-4-5 & 102.00 \\
\hline HDAT & (null) & S104-RISER-4-6 & 126.00 \\
\hline HDAT & (null) & S104-RISER-4-7 & 150.00 \\
\hline HDAT & (null) & S104-RISER-4-8 & 174.00 \\
\hline HDAT & (null) & S104-RISER-4-9 & 198.00 \\
\hline HDAT & (null) & S105-RISER-4-1 & 6.72 \\
\hline HDAT & (null) & S105-RISER-4-10 & 222.72 \\
\hline HDAT & (null) & S105-RISER-4-11 & 246.72 \\
\hline HDAT & (null) & S105-RISER-4-12 & 270.72 \\
\hline HDAT & (null) & S105-RISER-4-13 & 318.72 \\
\hline HDAT & (null) & S105-RISER-4-14 & 366.72 \\
\hline HDAT & (null) & S105-RISER-4-2 & 30.72 \\
\hline HDAT & (null) & S105-RISER-4-3 & 54.72 \\
\hline HDAT & (null) & S105-RISER-4-4 & 78.72 \\
\hline HDAT & (nutl) & S105-RISER-4-5 & 102.72 \\
\hline HDAT & (null) & S105-RISER-4-6 & 126.72 \\
\hline HDAT & (null) & S105-RISER-4-7 & 150.72 \\
\hline HDAT & (null) & S105-RISER-4-8 & 174.72 \\
\hline HDAT & (null) & S105-RISER-4-9 & 198.72 \\
\hline
\end{tabular}




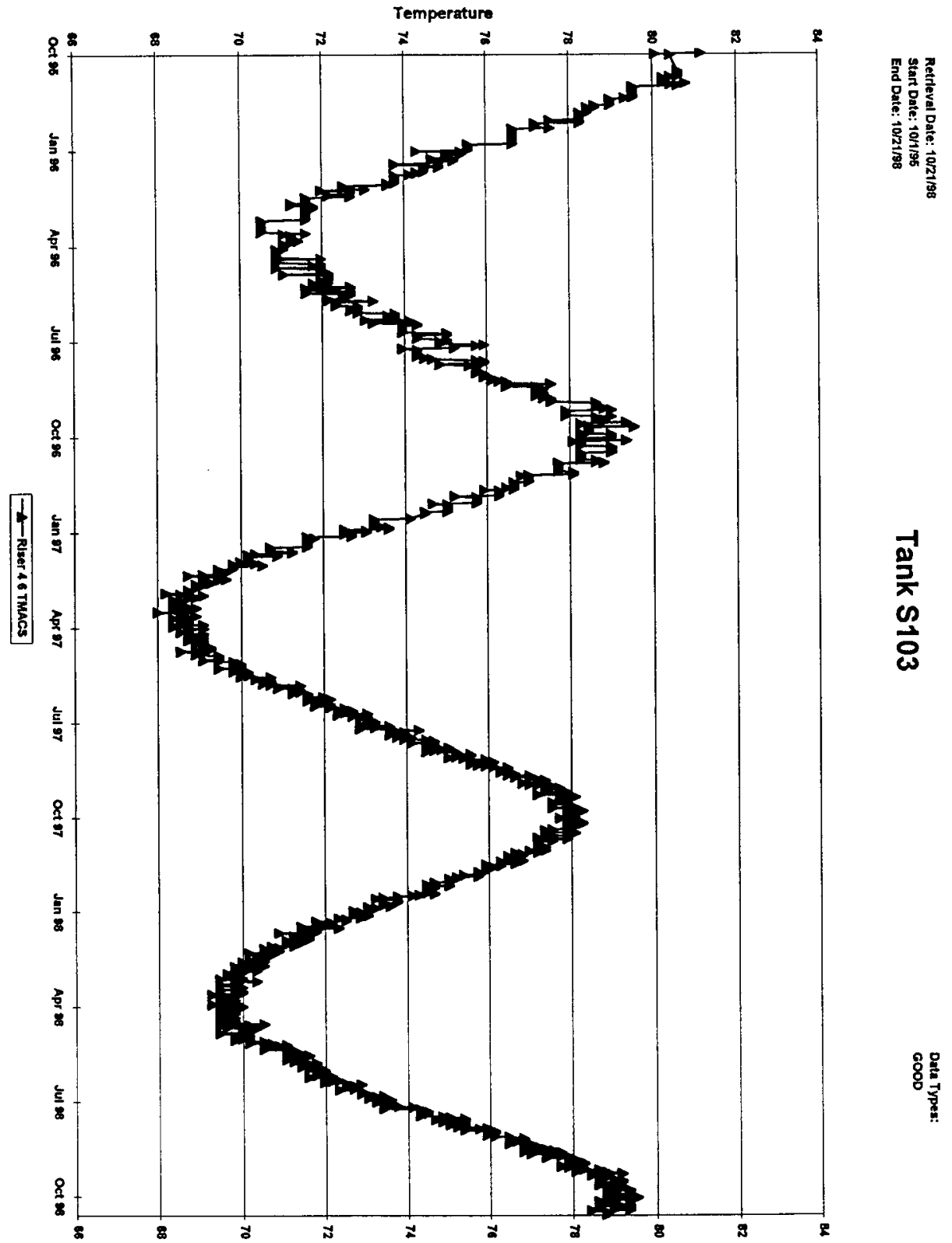


HNF-3776 REV. O PG. 30 OF 95

Surface Level (Inches)

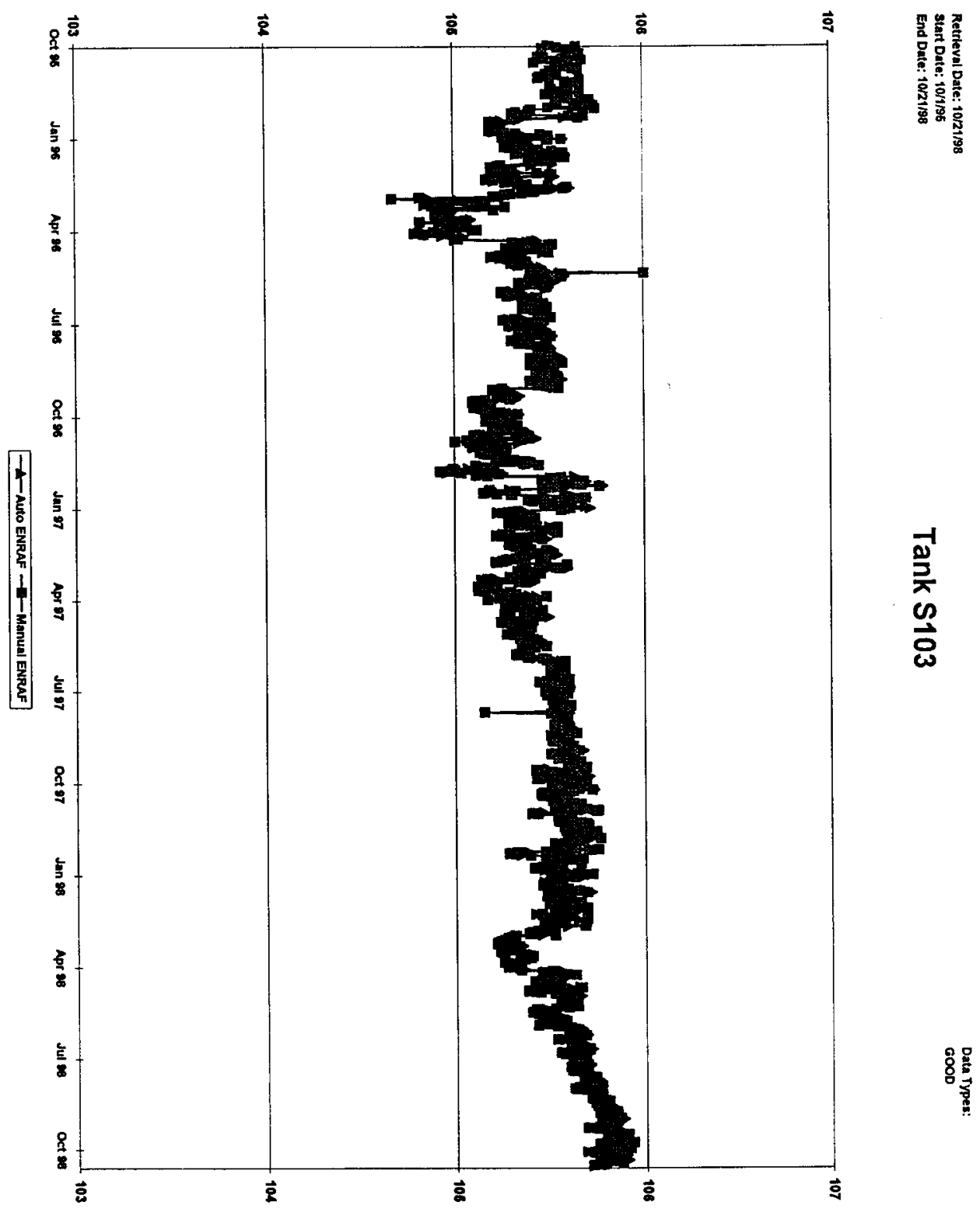


Sheet1

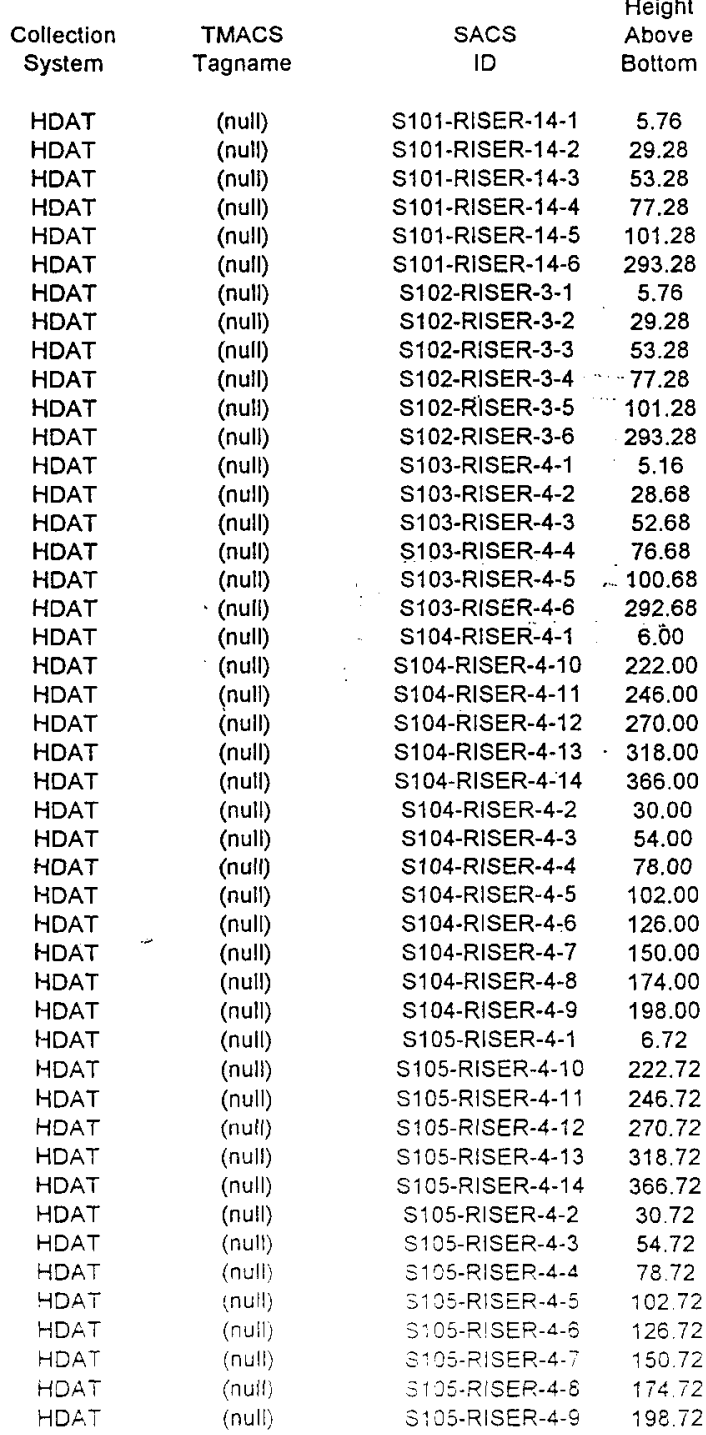




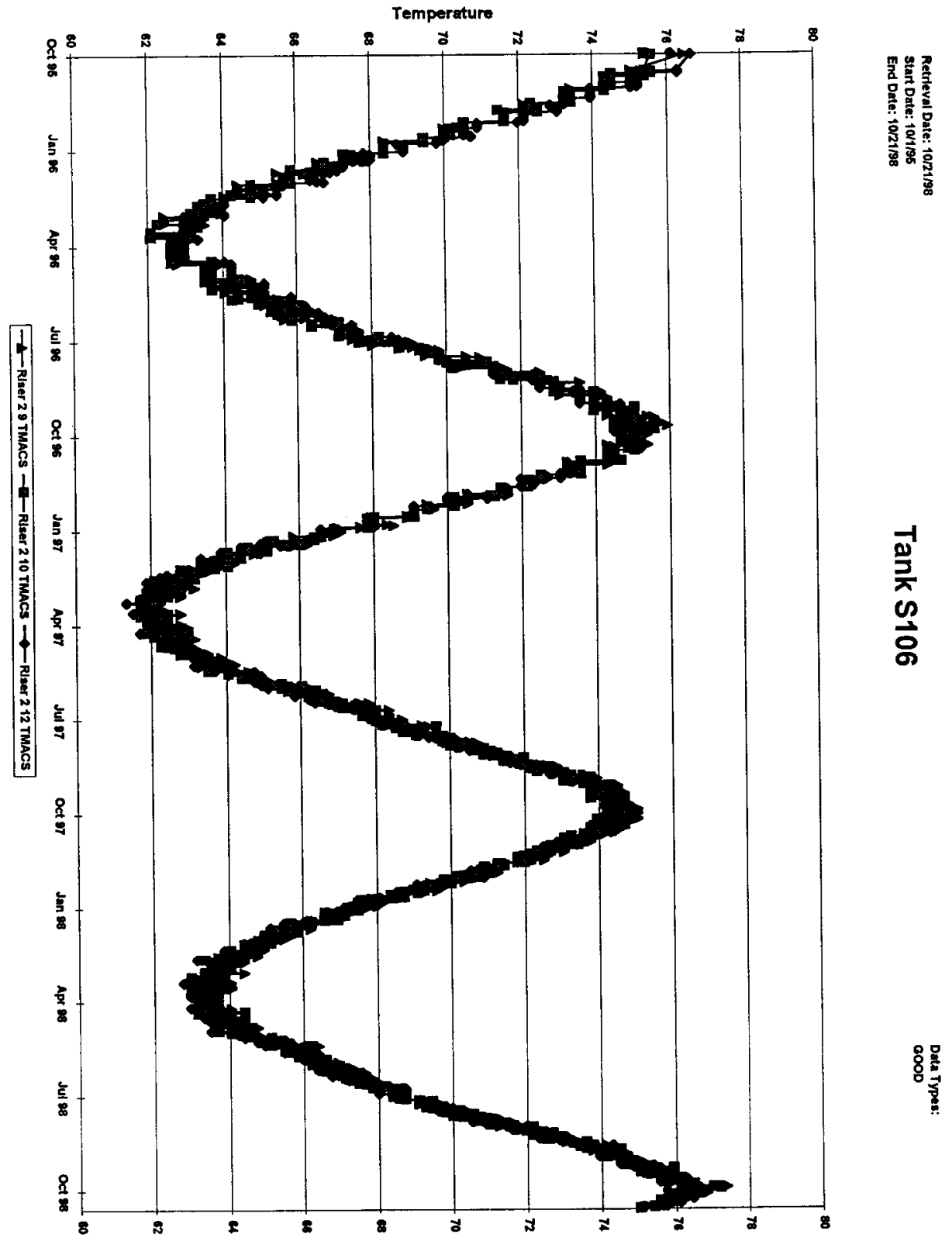


Surface Level (Inches)

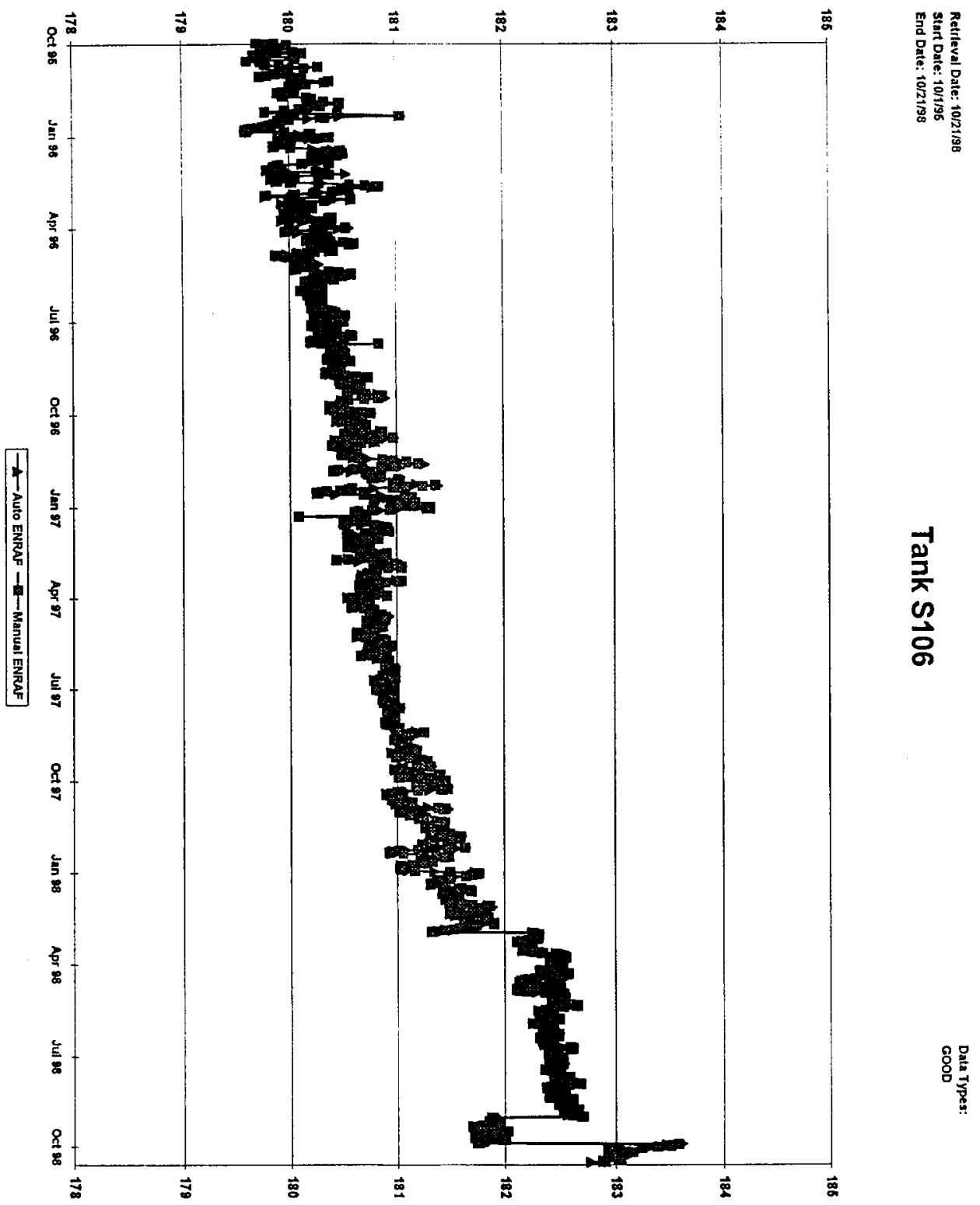


Sheet1

\begin{tabular}{|c|c|c|c|}
\hline HDAT & (null) & S106-RISER-2-1 & 5.52 \\
\hline HDAT & (null) & S106-RISER-2-10 & 221.52 \\
\hline HDAT & (null) & S106-RISER-2-11 & 245.52 \\
\hline HDAT & (nuli) & S106-RISER-2-12 & 269.52 \\
\hline HDAT & (null) & S106-RISER-2-13 & 317.52 \\
\hline HDAT & (nuli) & S106-RISER-2-14 & 365.52 \\
\hline HDAT & (nuli) & S106-RISER-2-2 & 29.52 \\
\hline HDAT & (null) & S106-RISER-2-3 & 53.52 \\
\hline HDAT & (null) & S106-RISER-2-4 & 77.52 \\
\hline HDAT & (null) & S106-RISER-2-5 & 101.52 \\
\hline HDAT & (null) & S106-RISER-2-6 & 125.52 \\
\hline HDAT & (null) & S106-RISER-2-7 & 149.52 \\
\hline HDAT & (null) & S106-RISER-2-8 & 173.52 \\
\hline HDAT & (nuil) & S106-RISER-2-9 & 197.52 \\
\hline HDAT & (null) & S107-RISER-4-1 & 5.76 \\
\hline HDAT & (null) & S107-RISER-4-2 & 29.28 \\
\hline HDAT & (null) & S107-RISER-4-3 & 53.28 \\
\hline HDAT & (nult) & S107-RISER-4-4 & 77.28 \\
\hline HDAT & (null) & S107-RISER-4-5 & 101.28 \\
\hline HDAT & (nuli) & S107-RISER-4-6 & 293.28 \\
\hline HDAT & (nuli) & S108-RISER-4-1 & 5.40 \\
\hline HDAT & (null) & S108-RISER-4-10 & 221.40 \\
\hline HDAT & (null) & S108-RISER-4-11 & 245.40 \\
\hline HDAT & (null) & S108-RISER-4-12 & 269.40 \\
\hline HDAT & (nuil) & S108-RISER-4-13 & 317.40 \\
\hline HDAT & (nuil) & S108-RISER-4-14 & 365.40 \\
\hline HDAT & (null) & S108-RISER-4-2 & 29.40 \\
\hline HDAT & (null) & S108-RISER-4-3 & 53.40 \\
\hline HDAT & (nuil) & S108-RISER-4-4 & 77.40 \\
\hline HDAT & (null) & S108-RISER-4-5 & 101.40 \\
\hline HDAT & (null) & S108-RISER-4-6 & 125.40 \\
\hline HDAT & (null) & S108-RISER-4-7 & 149.40 \\
\hline HDAT & (null) & S108-RISER-4-8 & 173.40 \\
\hline HDAT & (null) & S108-RISER-4-9 & 197.40 \\
\hline HDAT & (null) & S109-RISER-4-1 & 5.88 \\
\hline HDAT & (null) & S109-RISER-4-10 & 221.88 \\
\hline HDAT & (null) & S109-RISER-4-11 & 245.88 \\
\hline HDAT & (null) & S109-RISER-4-12 & 269.88 \\
\hline HDAT & (nuil) & S109-RISER-4-13 & 317.88 \\
\hline HDAT & (null) & S109-RISER-4-14 & 365.88 \\
\hline HDAT & (null) & S109-RISER-4-2 & 29.88 \\
\hline HDAT & (null) & S109-RISER-4-3 & 53.88 \\
\hline HDAT & (null) & S109-RISER-4-4 & 77.88 \\
\hline HDAT & (null) & S109-RISER-4-5 & 101.88 \\
\hline HDAT & (null) & S109-RISER-4-6 & 125.88 \\
\hline HDAT & (null) & S109-RISER-4-7 & 149.88 \\
\hline HDAT & (null) & S109-RISER-4-8 & 173.88 \\
\hline HDAT & (null) & S109-RISER-4-9 & 197.88 \\
\hline HDAT & (null) & S110-RISER-4-1 & 5.64 \\
\hline HDAT & (nuli) & S110-RISER-4-2 & 29.16 \\
\hline HDAT & (nuli) & S110-RISER-4-3 & 53.16 \\
\hline
\end{tabular}


HNF-3776 REV. O PG. 35 OF 95

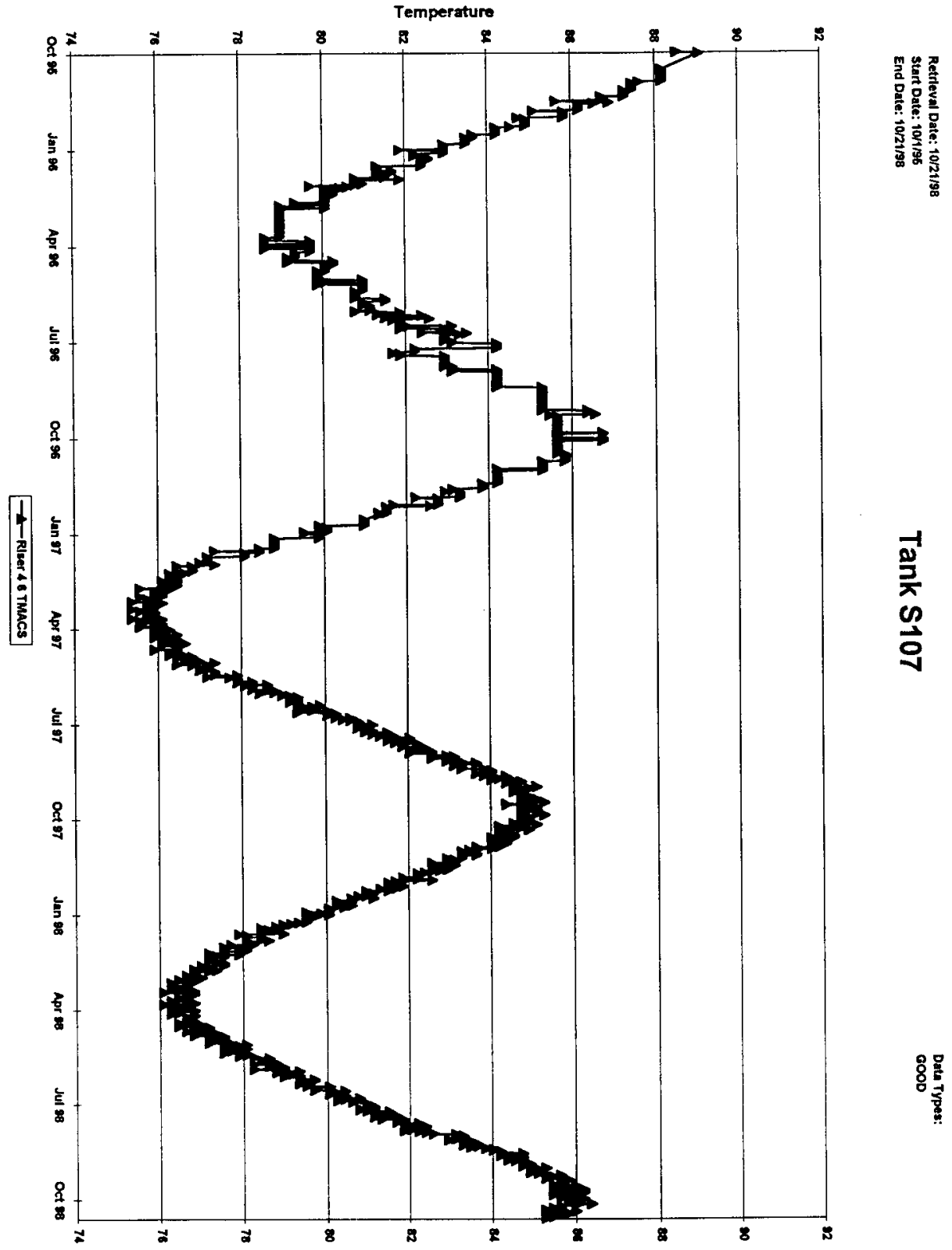




$$
1
$$


Sheet1

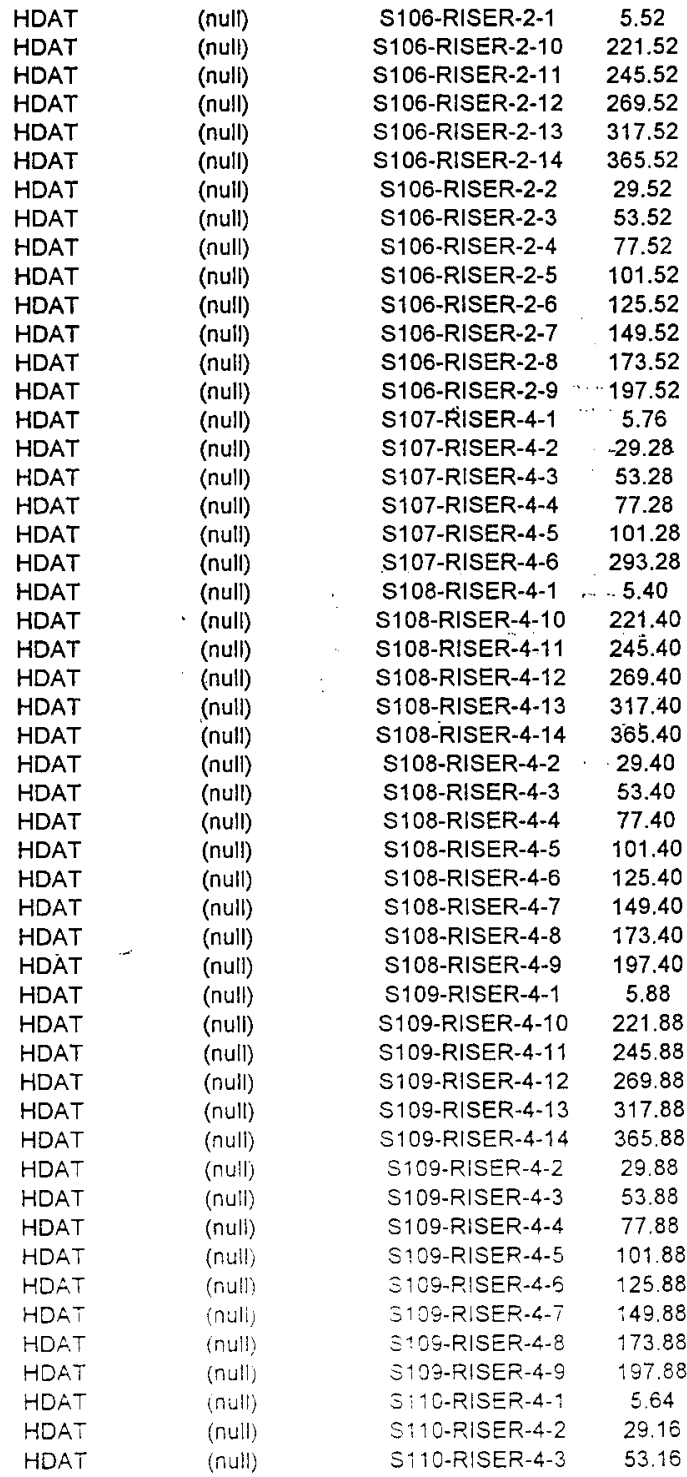




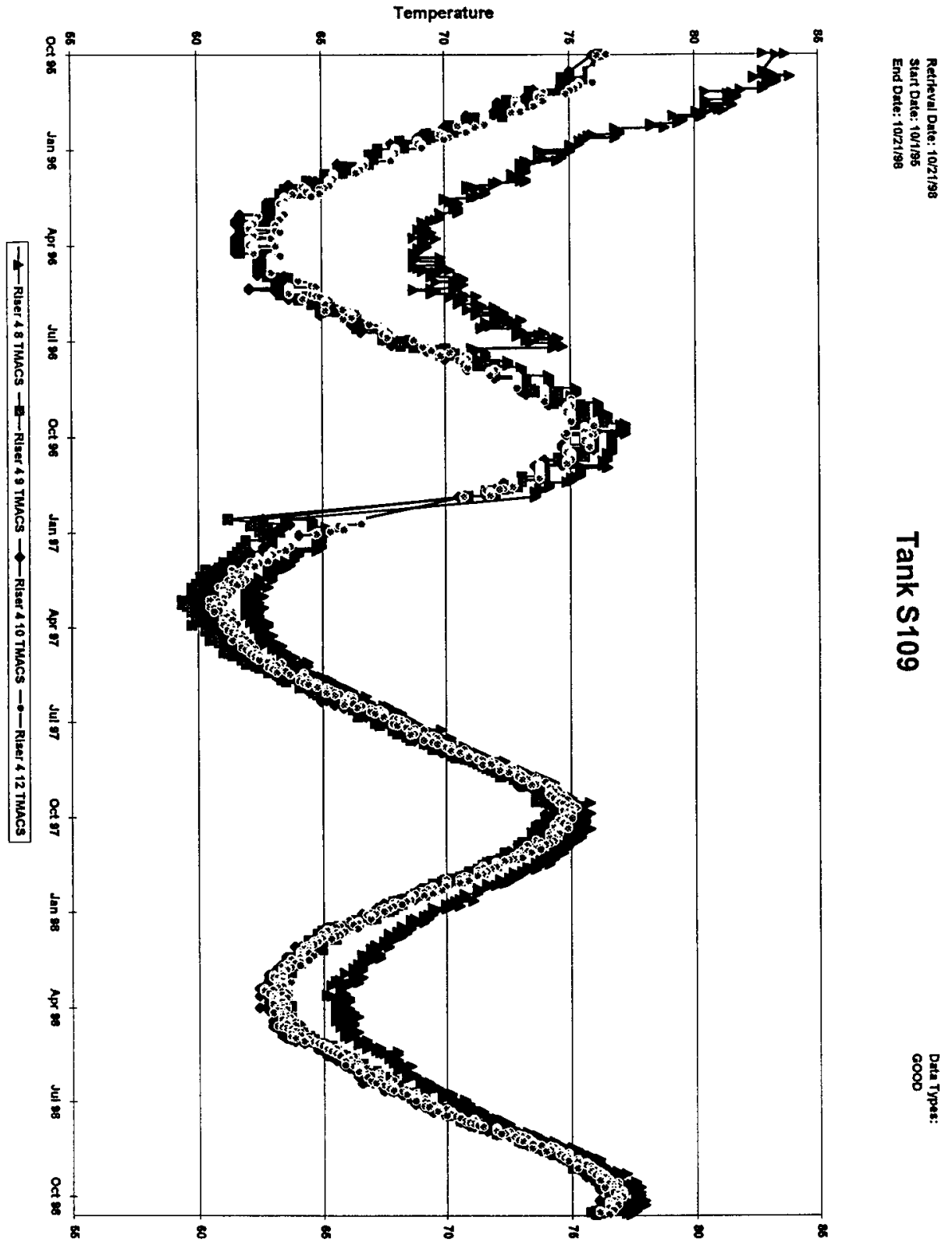


HNF-3776 REV. O PG. 39 OF 95

Surtace Level (Inches)

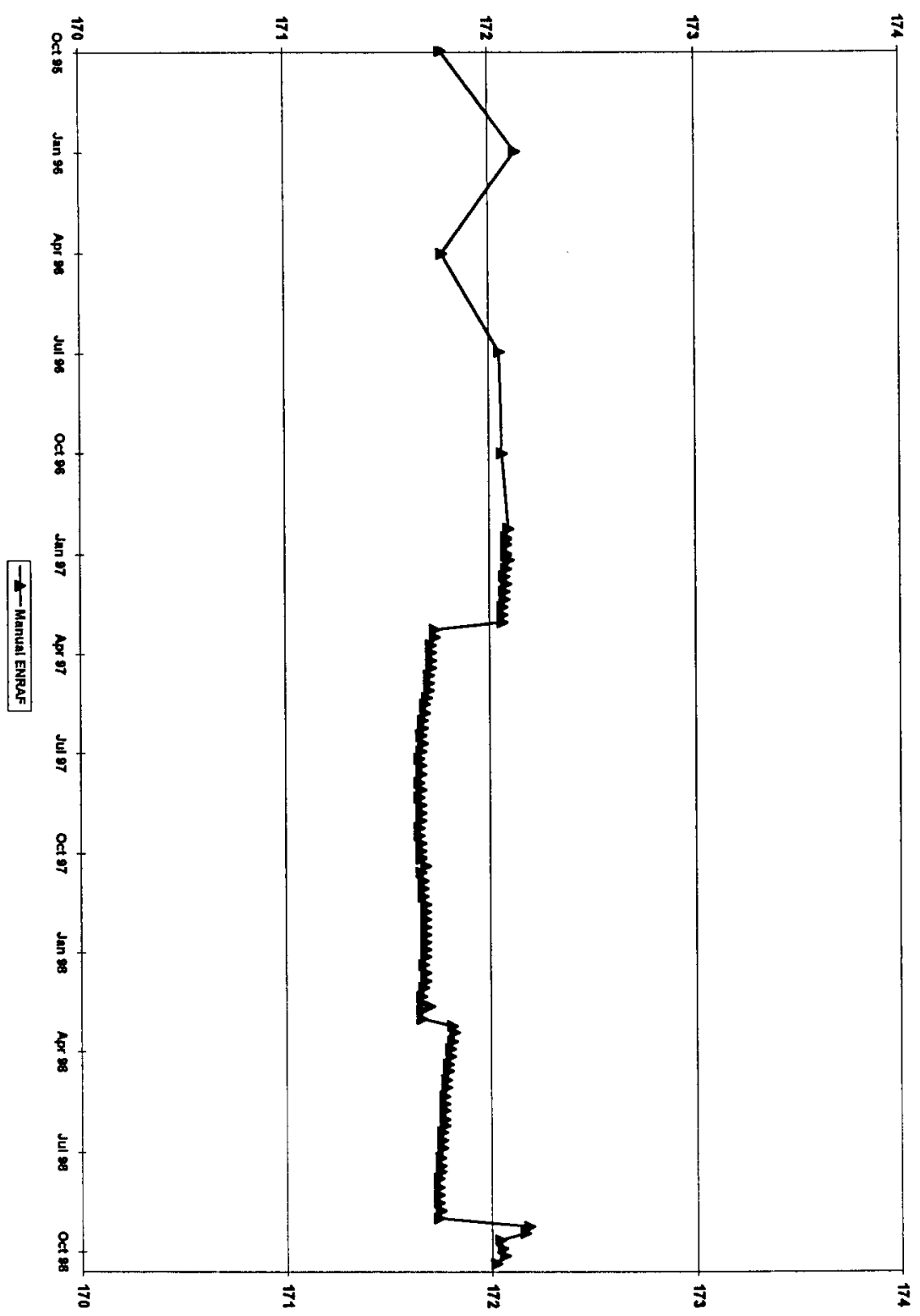

멋
옹
웅 
Sheet1

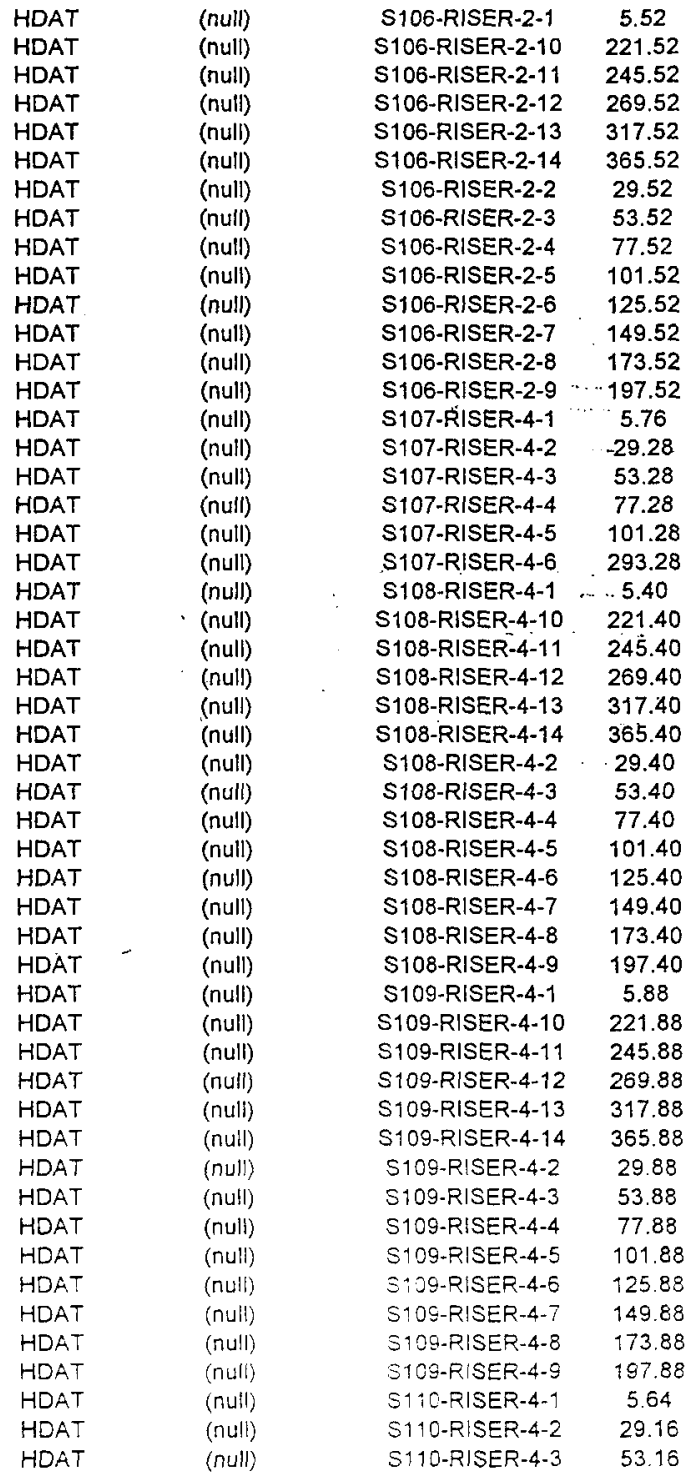


HNF-3776 REV. O PG. 41 OF 95

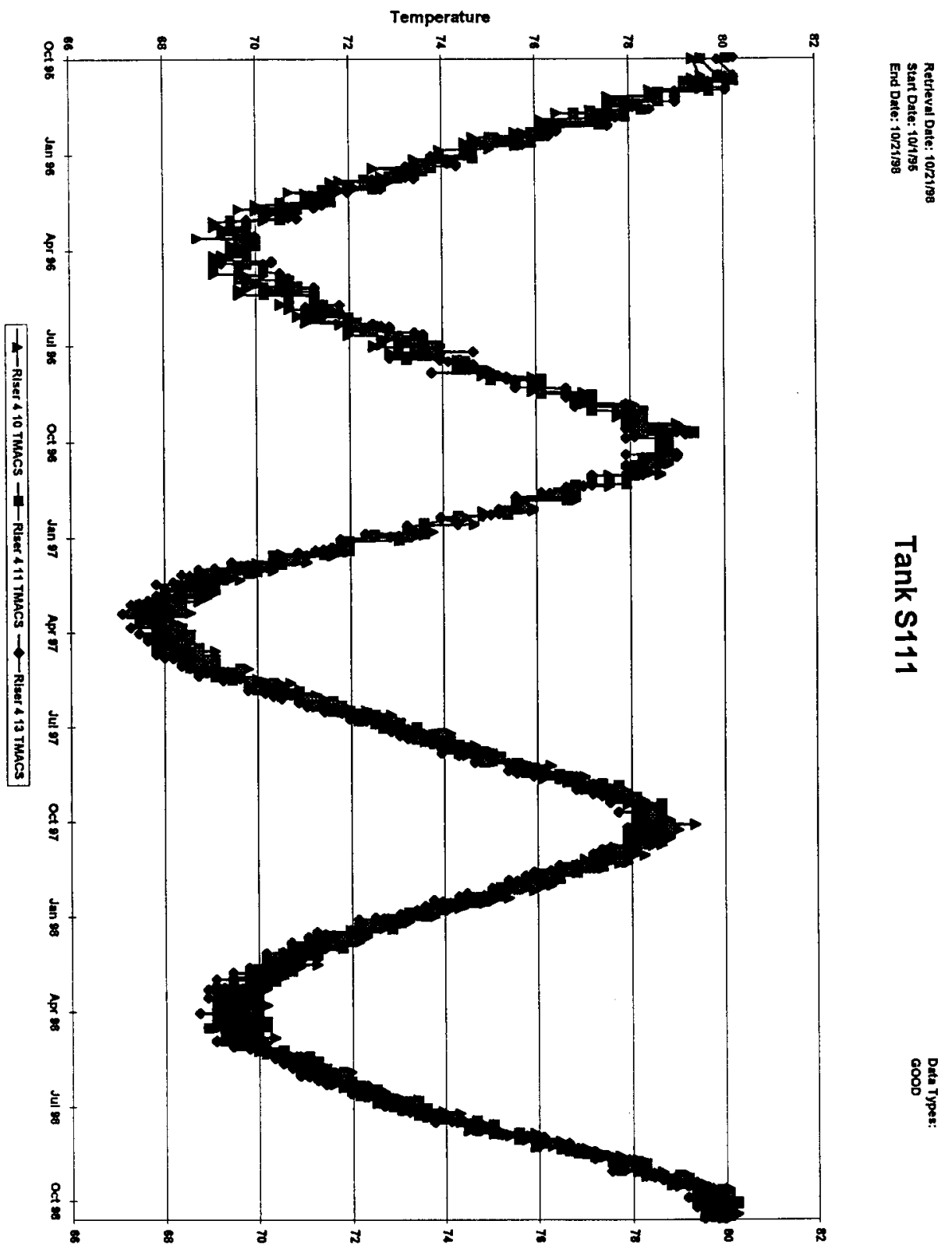


HNF-3776 REV. O PG. 42 OF 95

Surface Level (inches)

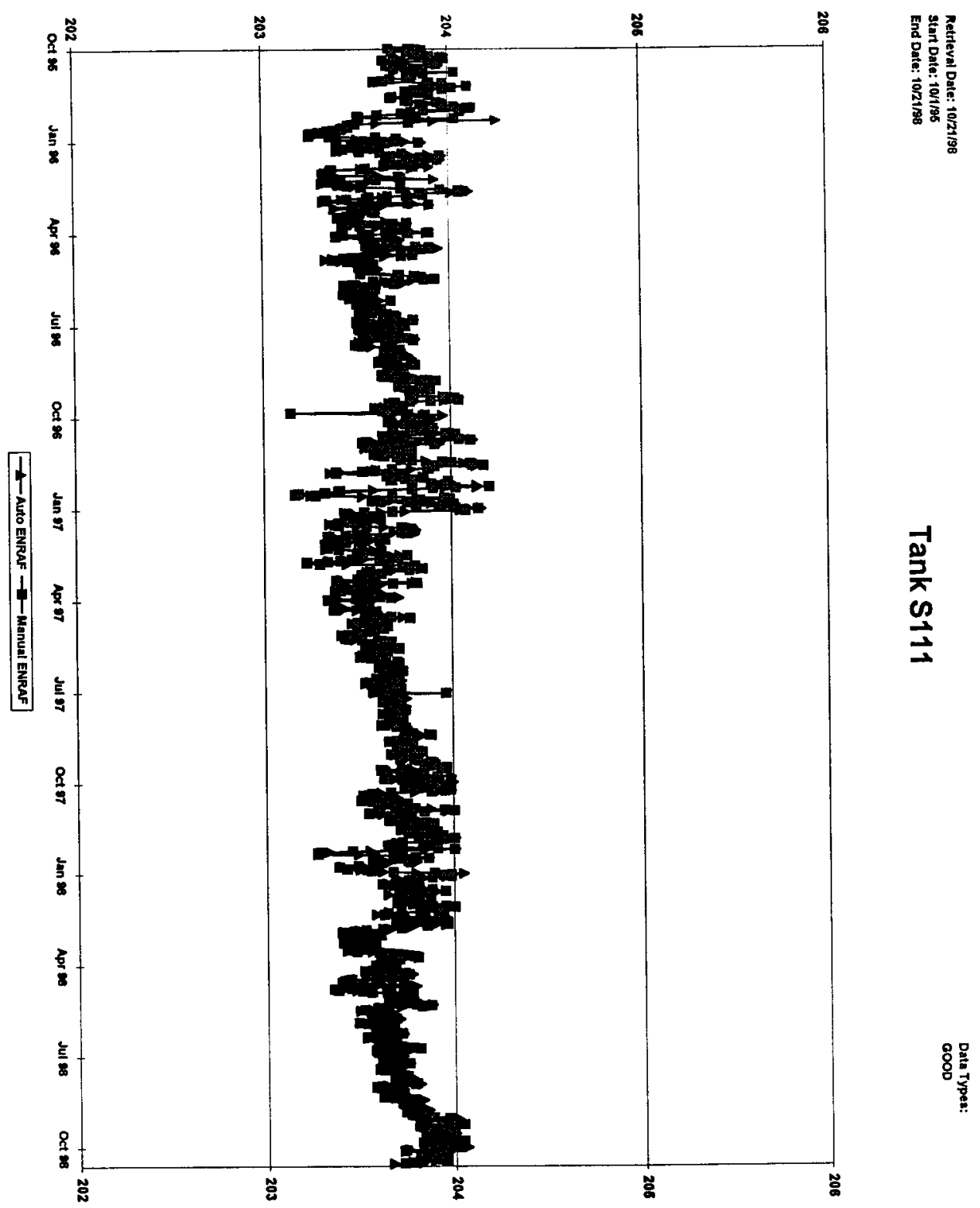


Sheet1

\begin{tabular}{|c|c|c|c|}
\hline HDAT & (null) & S110-RISER-4-4 & 77.16 \\
\hline HDAT & (null) & S110-RISER-4-5 & 101.16 \\
\hline HDAT & (nuli) & S110-RISER-4-6 & 293.16 \\
\hline HDAT & (null) & S111-RISER-4-1 & 6.00 \\
\hline HDAT & (null) & S111-RISER-4-10 & 222.00 \\
\hline HDAT & (null) & S111-RISER-4-11 & 246.00 \\
\hline HDAT & (null) & S111-RISER-4-12 & 270.00 \\
\hline HDAT & (null) & S111-RISER-4-13 & 318.00 \\
\hline HDAT & (nuli) & S111-RISER-4-14 & 366.00 \\
\hline HDAT & (null) & S111-RISER-4-2 & 30.00 \\
\hline HDAT & (nuil) & S111-RISER-4-3 & 54.00 \\
\hline HDAT & (nuil) & S111-RISER-4-4 & 78.00 \\
\hline HDAT & (null) & S111-RISER-4-5 & 102.00 \\
\hline HDAT & (null) & S111-RISER-4-6 & 126.00 \\
\hline HDAT & (nuli) & S111-RISER-4-7 & 150.00 \\
\hline HDAT & (null) & S111-RISER-4-8 & 174.00 \\
\hline HDAT & (null) & S111-RISER-4-9 & 198.00 \\
\hline HDAT & (null) & S112-RISER-4-1 & 6.00 \\
\hline HDAT & (null) & S112-RISER-4-10 & 222.00 \\
\hline HDAT & (null) & S112-RISER-4-11 & 246.00 \\
\hline HDAT & (nuli) & S112-RISER-4-12 & 270.00 \\
\hline HDAT & (null) & S112-RISER-4-13 & 318.00 \\
\hline HDAT & (null) & S112-RISER-4-14 & 366.00 \\
\hline HDAT & (null) & S112-RISER-4-2 & 30.00 \\
\hline HDAT & (null) & S112-RISER-4-3 & 54.00 \\
\hline HDAT & (null) & S112-RISER-4-4 & 78.00 \\
\hline HDAT & (null) & S112-RISER-4-5 & 102.00 \\
\hline HDAT & (nuli) & S112-RISER-4-6 & 126.00 \\
\hline HDAT & (null) & S112-RISER-4-7 & 150.00 \\
\hline HDAT & (null) & S112-RISER-4-8 & 174.00 \\
\hline HDAT & (nuli) & S112-RISER-4-9 & 198.00 \\
\hline HDAT & (null) & SX101-RISER-15-1 & 20.52 \\
\hline HDAT & (nuil) & SX101-RISER-15-2 & 44.04 \\
\hline HDAT & (null) & SX101-RISER-15-3 & 68.04 \\
\hline HDAT & (null) & SX101-RISER-15-4 & 92.04 \\
\hline HDAT & (null) & SX101-RISER-15-5 & 116.04 \\
\hline HDAT & (null) & SX101-RISER-15-6 & 383.04 \\
\hline HDAT & (null) & SX102-RISER-16-1 & 17.88 \\
\hline HDAT & (null) & SX102-RISER-16-2 & 41.40 \\
\hline HDAT & (null) & SX102-RISER-16-3 & 65.40 \\
\hline HDAT & (null) & SX102-RISER-16-4 & 89.40 \\
\hline HDAT & (null) & SX102-RISER-16-5 & 113.40 \\
\hline HDAT & (null) & SX102-RISER-16-6 & 377.40 \\
\hline HDAT & (null) & SX103-RISER-2-1 & 4.08 \\
\hline HDAT & (null) & S×103-RISER-2-2 & 27.60 \\
\hline HDAT & (nuli) & SX103-RISER-2-3 & 51.60 \\
\hline HDAT & (null) & SX103-RISER-2-4 & 75.60 \\
\hline HDAT & (null) & S×103-RISER-2-5 & 99.60 \\
\hline HDAT & (nuli) & SX103-RISER-2-6 & 366.60 \\
\hline
\end{tabular}


HNF-3776 REV. O PG. 44 OF 95

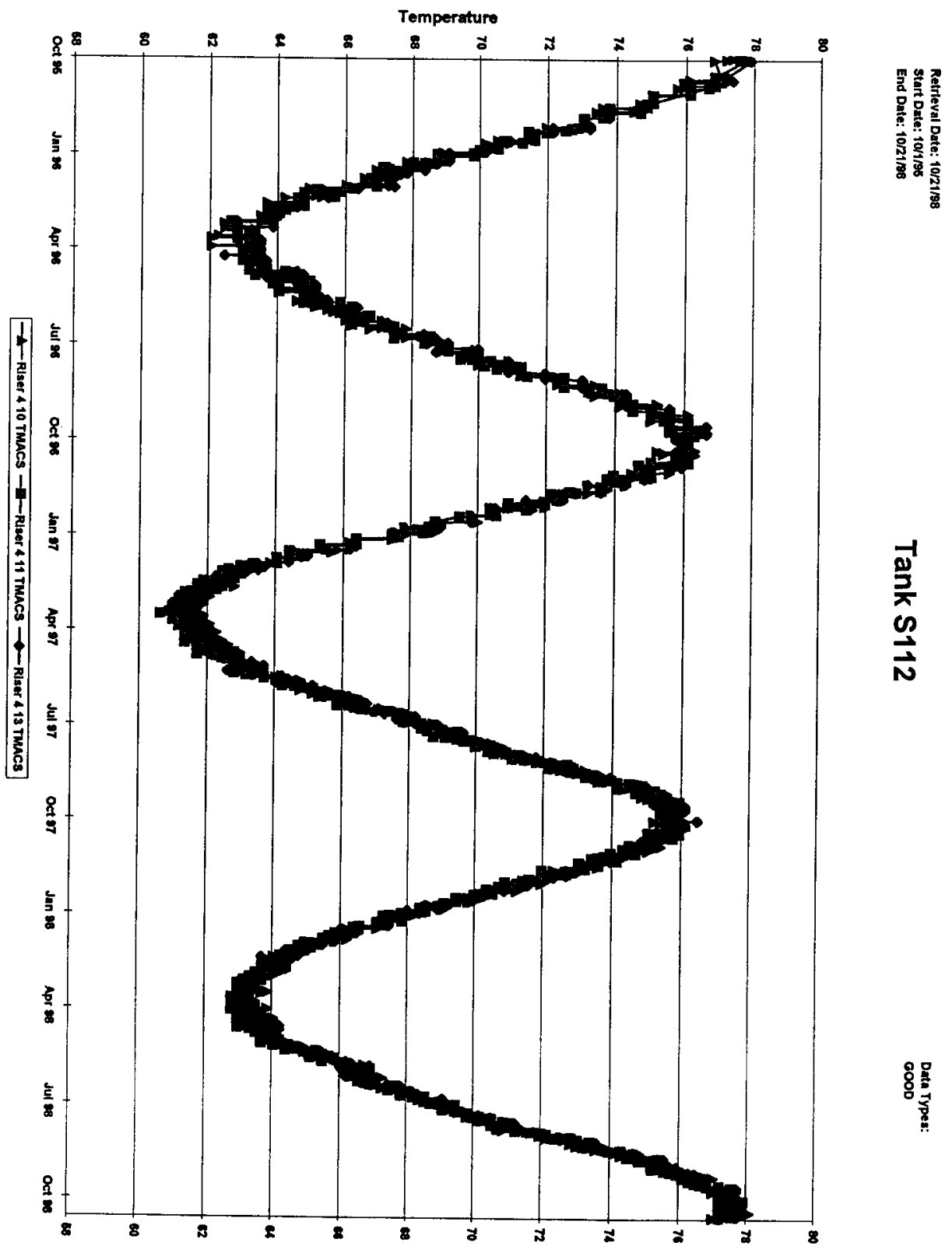




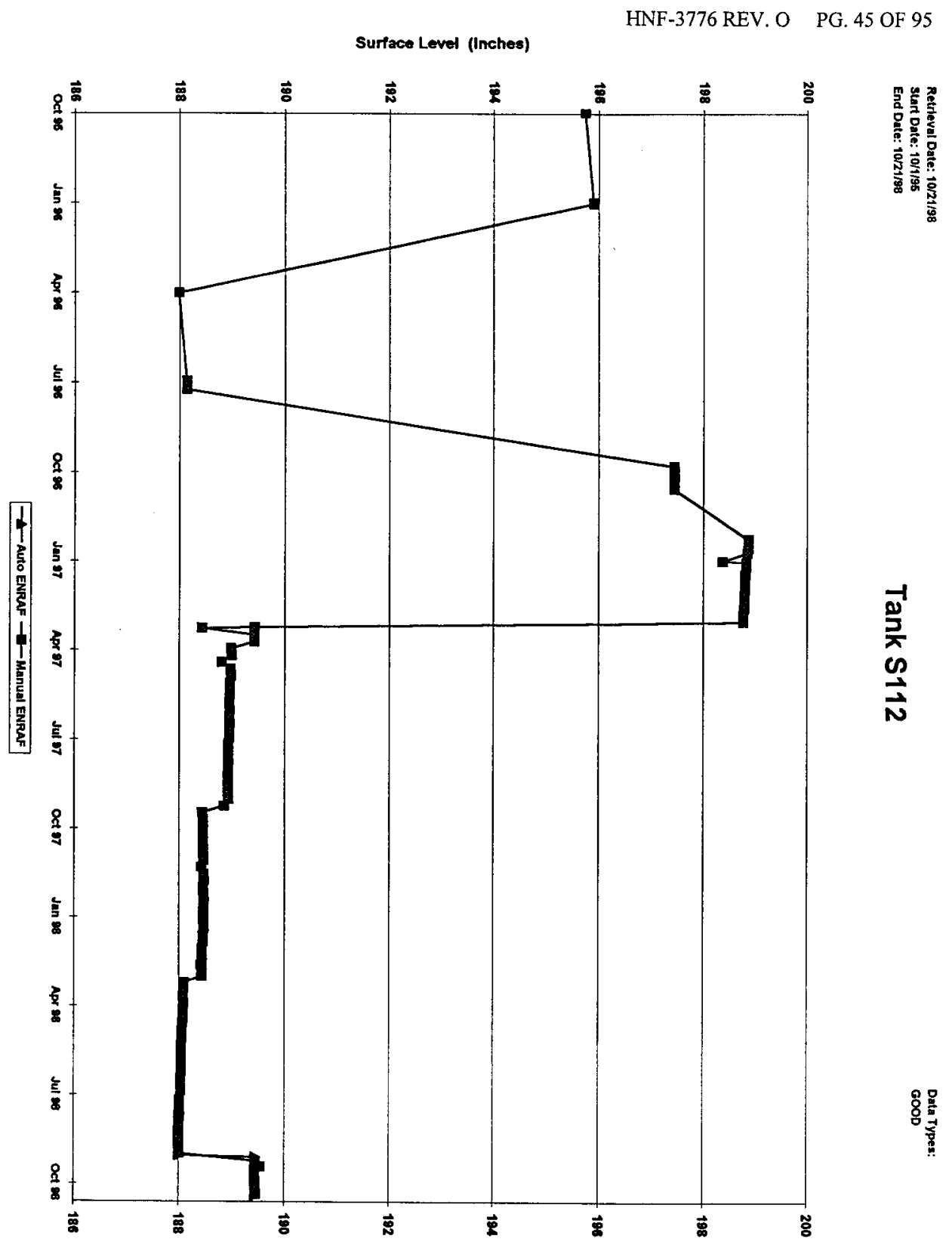


Sheet1

\begin{tabular}{|c|c|c|}
\hline \multicolumn{3}{|l|}{ HDAT } \\
\hline HDAT & & (null) \\
\hline HOAT & & (nu!l) \\
\hline HDAT & & (null) \\
\hline HDAT & & (nuli) \\
\hline HDAT & & (null) \\
\hline HDAT & & (null) \\
\hline HDAT & & (null) \\
\hline HDAT & & (null) \\
\hline HDAT & & (null) \\
\hline HDAT & & (null) \\
\hline HDAT & & (null) \\
\hline HDAT & & (null) \\
\hline HDAT & & (null) \\
\hline HDAT & & (null) \\
\hline HDAT & & (null) \\
\hline HDAT & & (null) \\
\hline HDAT & & (null) \\
\hline HDAT & & (null) \\
\hline HDAT & & (null) \\
\hline HDAT & & (null) \\
\hline HDAT & & (null) \\
\hline HDAT & & (null) \\
\hline HDAT & & (null) \\
\hline HDAT & & (null) \\
\hline HDAT & & (null) \\
\hline HDAT & & (null) \\
\hline HDAT & & (null) \\
\hline HDAT & & (null) \\
\hline HDAT & & (nuil) \\
\hline HDAT & & (null) \\
\hline HDAT & & (null) \\
\hline HDAT & & (nuli) \\
\hline HDÄT & - & (null) \\
\hline HDAT & & (nuil) \\
\hline HDAT & & (null) \\
\hline HDAT & & (null) \\
\hline HDAT & & (null) \\
\hline HDAT & & (null) \\
\hline HDAT & & (nuli) \\
\hline HDAT & & (nuil) \\
\hline HDAT & & (null) \\
\hline HDAT & & (null) \\
\hline HDAT & & (null) \\
\hline HDAT & & (null) \\
\hline HDAT & & (nuli) \\
\hline HDAT & & (nully \\
\hline HDAT & & (null) \\
\hline HDAT & & (nuli) \\
\hline
\end{tabular}

\begin{tabular}{|c|c|}
\hline & \\
\hline 110-RISEF & \\
\hline 110-RISER & \\
\hline & \\
\hline 10 & \\
\hline & \\
\hline & \\
\hline & \\
\hline SER & 660 \\
\hline SER & \\
\hline ER & \\
\hline ER & 8.00 \\
\hline SER-4-5 & 02.00 \\
\hline SER-4-6 & \\
\hline SER-4-7 & \\
\hline $4-8$ & \\
\hline SEF & \\
\hline SEF & \\
\hline SER & \\
\hline SER & \\
\hline 12 & \\
\hline & \\
\hline & \\
\hline & \\
\hline & \\
\hline-4 & \\
\hline $4-5$ & \\
\hline & \\
\hline & \\
\hline ER-4-8 & \\
\hline S11 & \\
\hline ISEF & \\
\hline 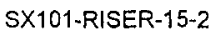 & \\
\hline-3 & \\
\hline 15.4 & \\
\hline FR & \\
\hline 5 & \\
\hline 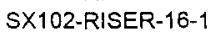 & \\
\hline ISER & \\
\hline RISER & \\
\hline ISER & \\
\hline SER & \\
\hline 10 & \\
\hline ISER-2-1 & \\
\hline$E R-2-2$ & 27. \\
\hline RISER-2-3 & 51.6 \\
\hline$\times 103-R I S E R-2-4$ & 75.6 \\
\hline ER-2-5 & \\
\hline$-2-6$ & \\
\hline
\end{tabular}


HNF-3776 REV. O PG. 47 OF 95

Temperature

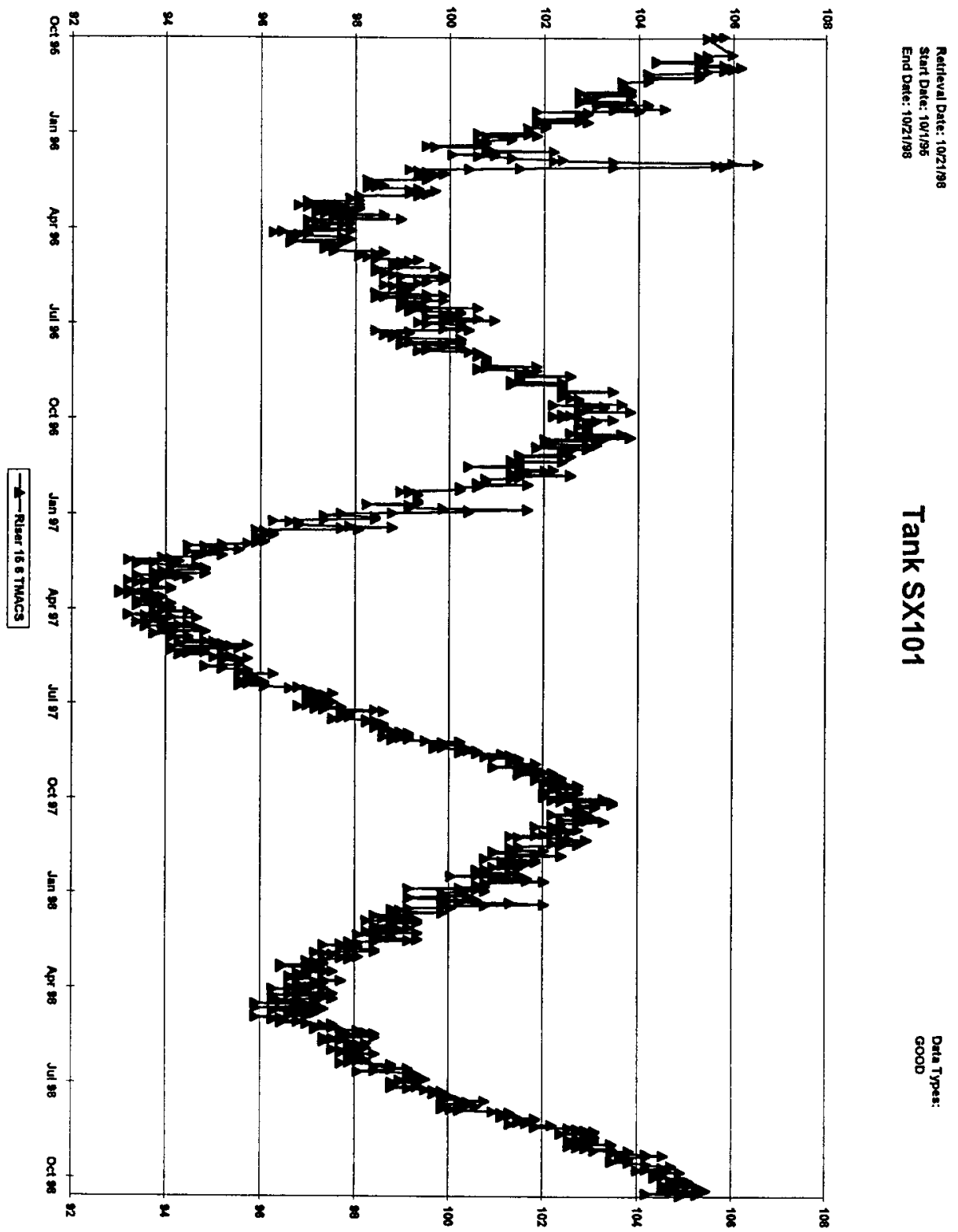




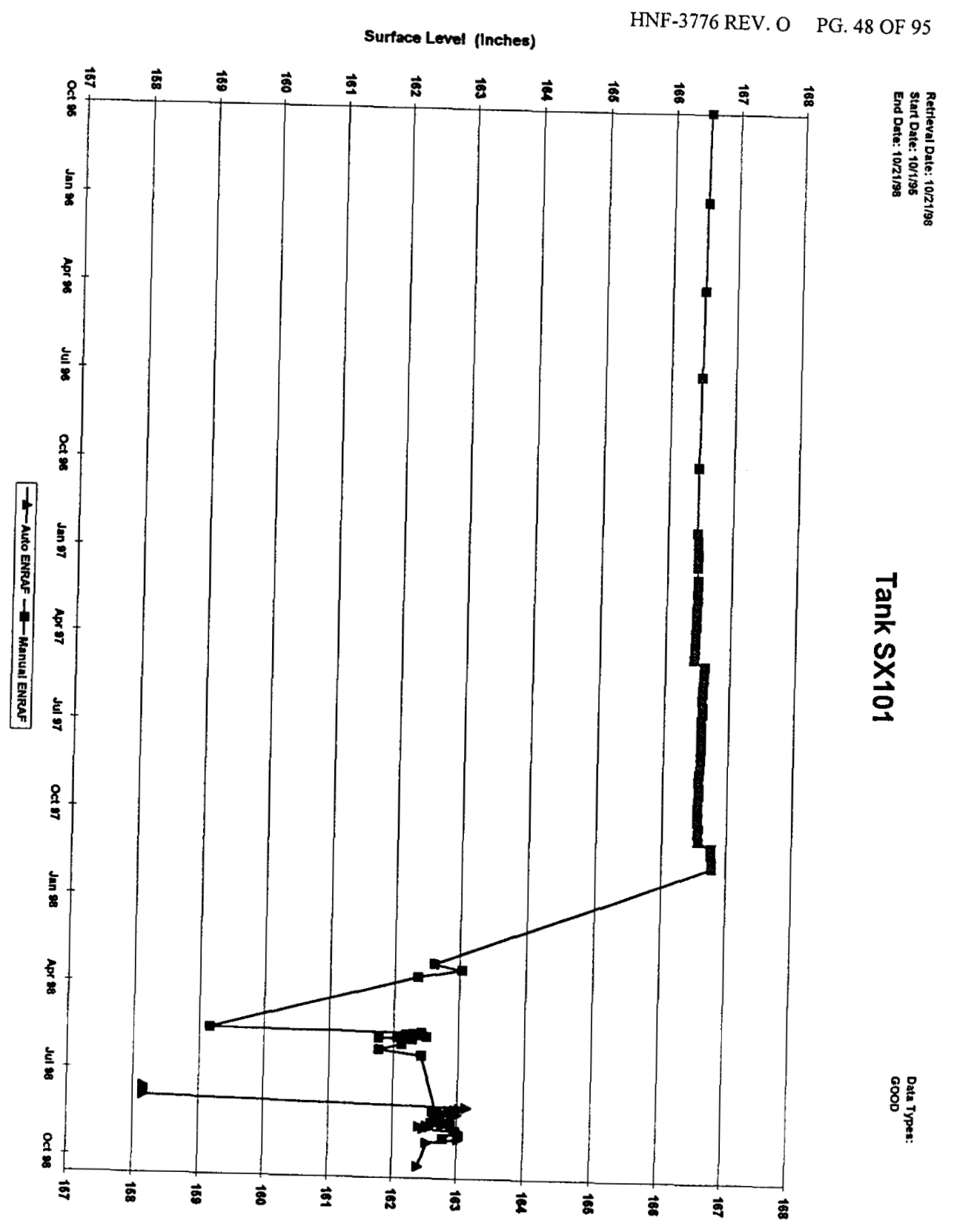




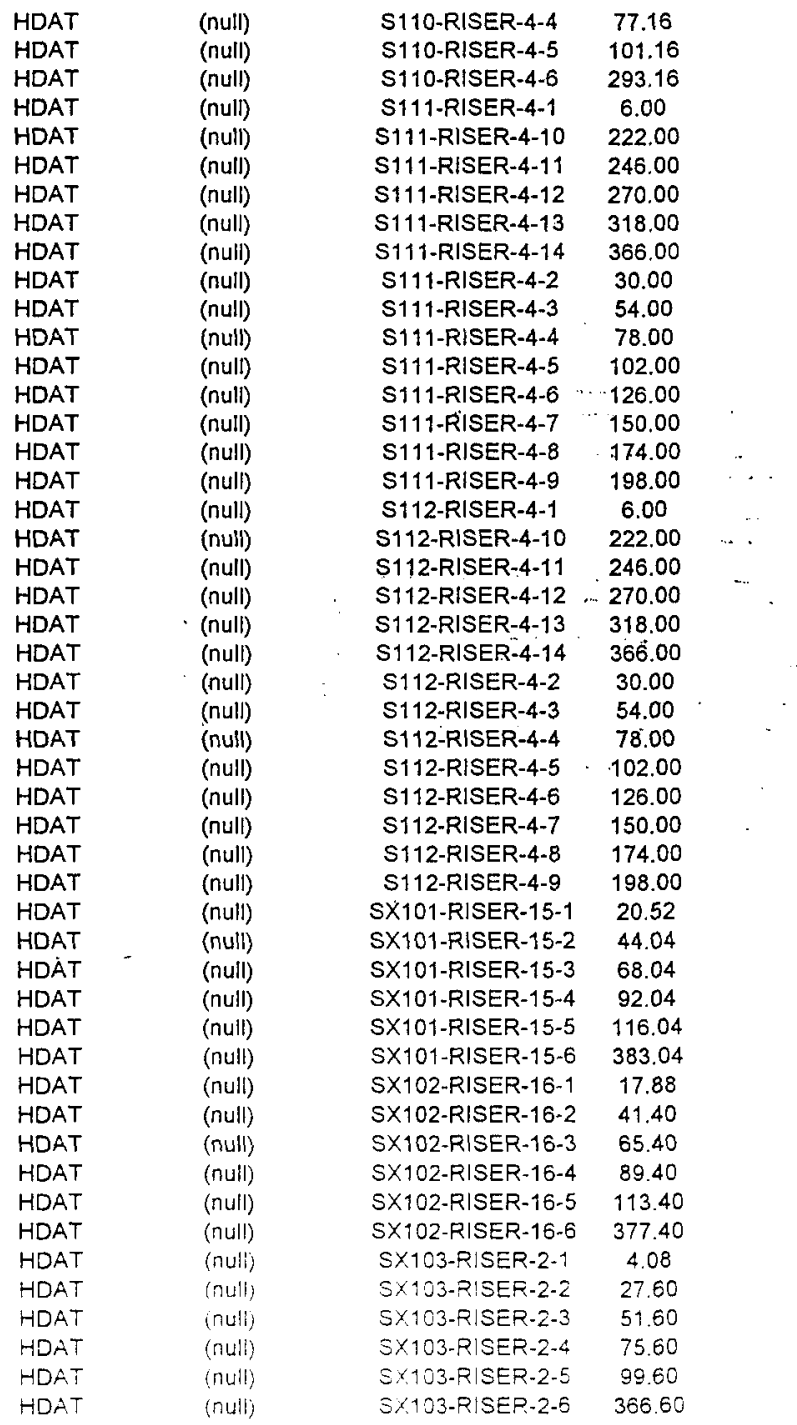


HNF-3776 REV. O PG. 50 OF 95

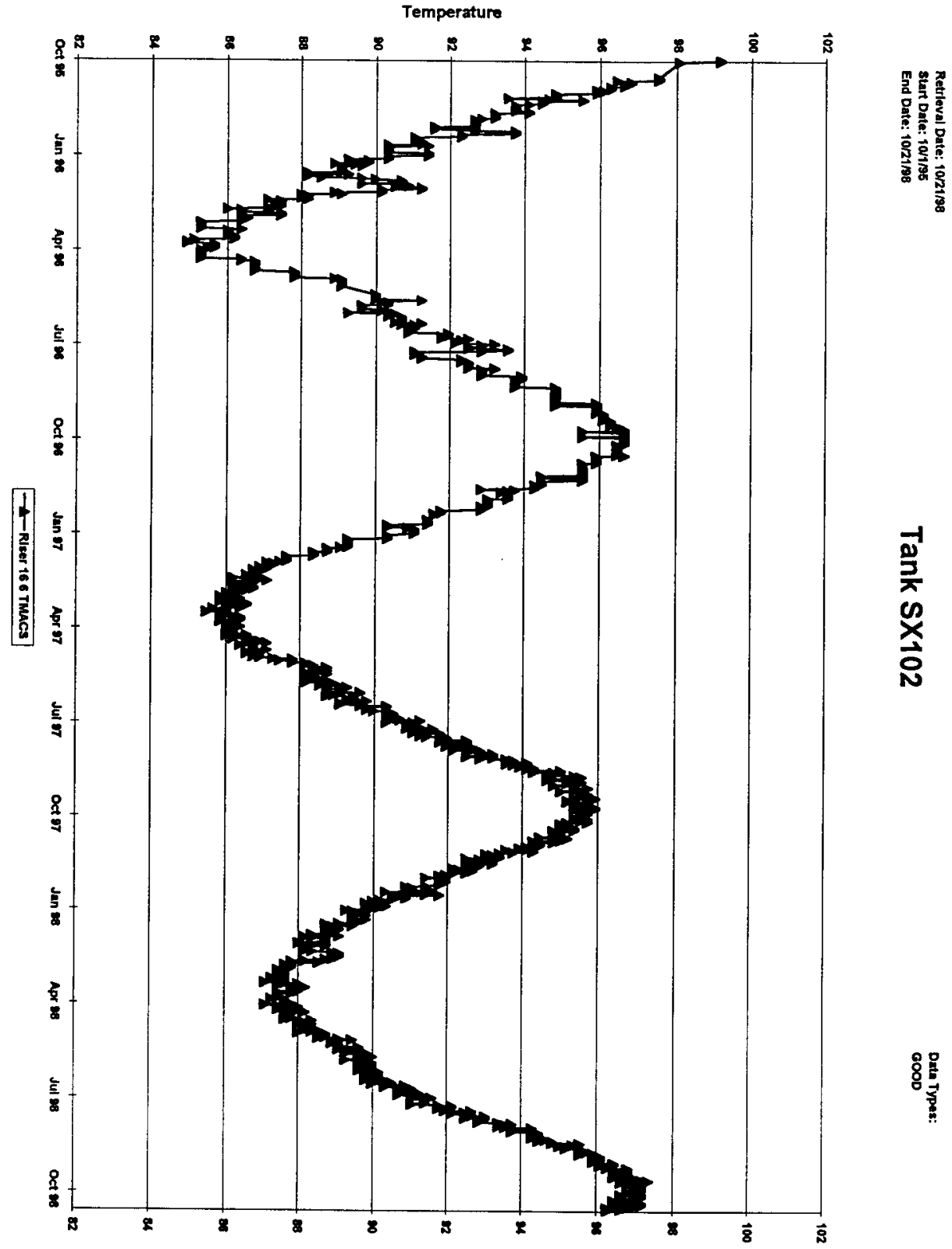


Surface Level (Inches)

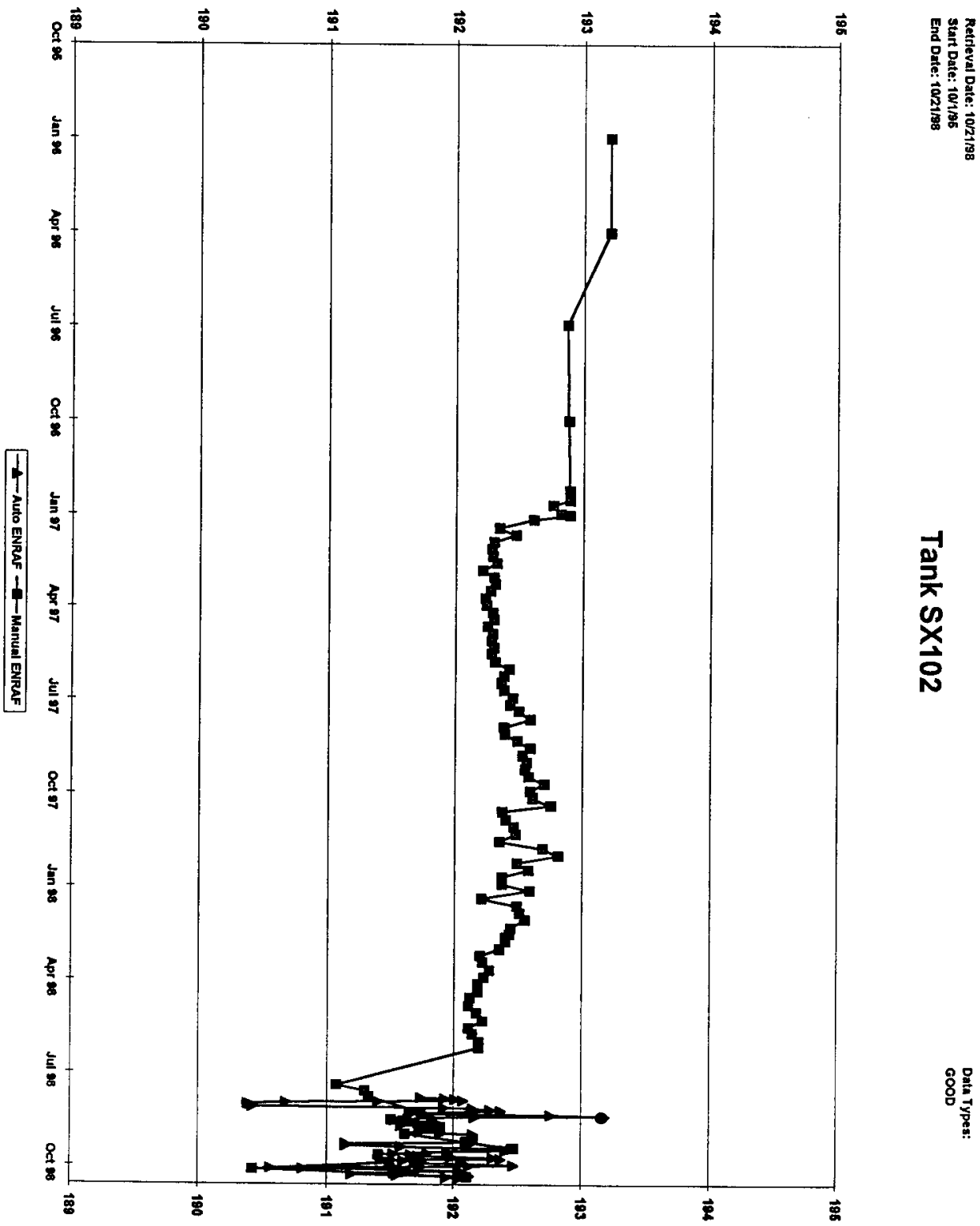


Sheet1

\begin{tabular}{|c|c|c|c|c|}
\hline HDAT & & (nuli) & S110-RISER-4-4 & 77.16 \\
\hline HDAT & & (null) & S110-RISER-4-5 & 101.16 \\
\hline HDAT & & (null) & S110-RISER-4-6 & 293.16 \\
\hline HDAT & & (null) & S111-RISER-4-1 & 6.00 \\
\hline HDAT & & (null) & S111-RISER-4-10 & 222.00 \\
\hline HDAT & & (null) & S111-RISER-4-11 & 246.00 \\
\hline HDAT & & (null) & S111-RISER-4-12 & 270.00 \\
\hline HDAT & & (null) & S111-RISER-4-13 & 318.00 \\
\hline HDAT & & (null) & S111-RISER-4-14 & 366.00 \\
\hline HDAT & & (null) & S111-RISER-4-2 & 30.00 \\
\hline HDAT & & (null) & S111-RISER-4-3 & 54.00 \\
\hline HDAT & & (null) & S111-RISER-4-4 & 78.00 \\
\hline HDAT & & (nult) & S111-RISER-4-5 & 102.00 \\
\hline HDAT & & (nuli) & S111-RISER-4-6 & $\cdots 126.00$ \\
\hline HDAT & & (null) & S111-RISSER-4-7 & 150.00 \\
\hline HDAT & & (null) & S111-RISER-4-8 & .174 .00 \\
\hline HDAT & & (nuli) & S111-RISER-4-9 & 198.00 \\
\hline HDAT & & (null) & S112-RISER-4-1 & 6.00 \\
\hline HDAT & & (null) & S112-RISER-4-10 & 222.00 \\
\hline HDAT & & (null) & S112-RISER-4-11 & 246.00 \\
\hline HDAT & & (null) & S112-RISER-4-12 & $\ldots 270.00$ \\
\hline HDAT & & (null) & S112-RISER-4-13 & 318.00 \\
\hline HDAT & & (nuli) & S112-RISER-4-14 & 366.00 \\
\hline HDAT & & (nuil) & S112-RISER-4-2 & 30.00 \\
\hline HDAT & & (nuli) & S112-RISER-4-3 & 54.00 \\
\hline HDAT & & (null) & S†12-RISER-4-4 & 78.00 \\
\hline HDAT & & (null) & S112-RISER-4-5 & 102.00 \\
\hline HDAT & & (null) & S112-RISER-4-6 & 126.00 \\
\hline HDAT & & (nuli) & S112-RISER-4-7 & 150.00 \\
\hline HDAT & & (null) & S112-RISER-4-8 & 174.00 \\
\hline HDAT & & (nuil) & S112-RISER-4-9 & 198.00 \\
\hline HDAT & & (null) & SX101-RISER-15-1 & 20.52 \\
\hline HDAT & $\ldots$ & (null) & SX101-RISER-15-2 & 44.04 \\
\hline HDÄT & 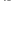 & (null) & SX101-RISER-15-3 & 68.04 \\
\hline HDAT & & (null) & SX101-RISER-15-4 & 92.04 \\
\hline HDAT & & (null) & SX101-RISER-15-5 & 116.04 \\
\hline HDAT & & (null) & SX101-RISER-15-6 & 383.04 \\
\hline HDAT & & (null) & SX102-RISER-16-1 & 17.88 \\
\hline HDAT & & (null) & SX102-RISER-16-2 & 41.40 \\
\hline HDAT & & (null) & SX102-RISER-16-3 & 65.40 \\
\hline HDAT & & (nuil) & SX102-RISER-16-4 & 89.40 \\
\hline HDAT & & (null) & SX102-RISER-16-5 & 113.40 \\
\hline HDAT & & (null) & SX102-RISER-16-6 & 377.40 \\
\hline HDAT & & $($ null) & SX103-RISER-2-1 & 4.08 \\
\hline HDAT & & (null) & SK-103-RISER-2-2 & 27.60 \\
\hline HDAT & & (nuil) & SX103-RISER-2-3 & 51.60 \\
\hline HDAT & & (null) & SX103-RISER-2.4 & 75.60 \\
\hline HDAT & & (null) & S×103-RISEP-2-5 & 99.60 \\
\hline HDAT & & (null) & $3 \times 103-R \mid S E R-2-6$ & 366.60 \\
\hline
\end{tabular}


HNF-3776 REV. O PG. 53 OF 95

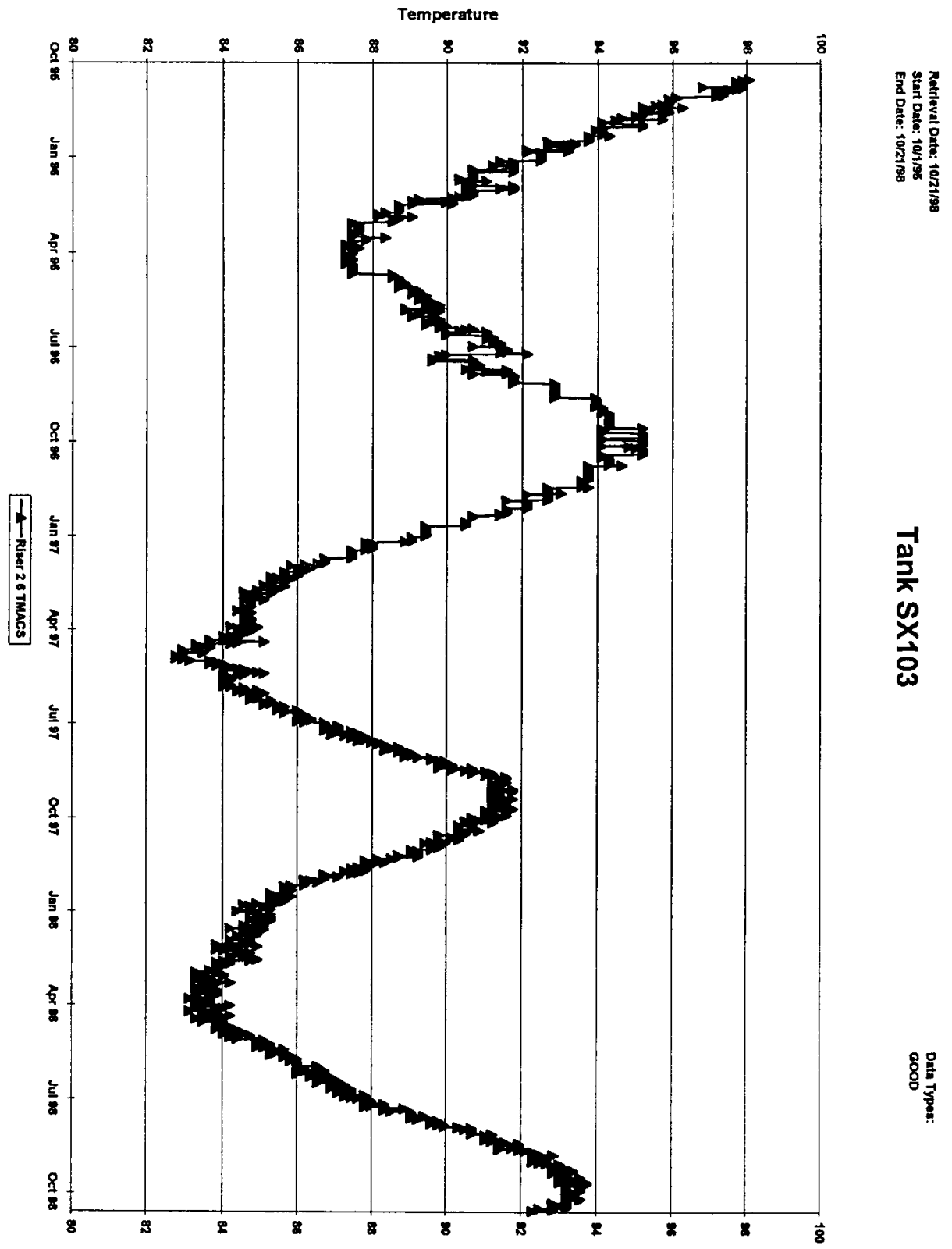


Sheet1

\begin{tabular}{|c|c|c|c|c|c|}
\hline HDAT & & (null) & & S110-RISER-4-4 & 77.16 \\
\hline HDAT & & (nuil) & & S110-RISER-4-5 & 101.16 \\
\hline HDAT & & (null) & & S110-RISER-4-6 & 293.16 \\
\hline HDAT & & (null) & & S111-RISER-4-1 & 6.00 \\
\hline HDAT & & (null) & & S111-RISER-4-10 & 222.00 \\
\hline HDAT & & (null) & & S111-RISER-4-11 & 246.00 \\
\hline HDAT & & (null) & & S111-RISER-4-12 & 270.00 \\
\hline HDAT & & (null) & & S111-RISER-4-13 & 318.00 \\
\hline HDAT & & (null) & & S111-RISER-4-14 & 366.00 \\
\hline HDAT & & (null) & & S111-RISER-4-2 & 30.00 \\
\hline HDAT & & (null) & & S111-RISER-4-3 & 54.00 \\
\hline HDAT & & (null) & & S111-RISER-4-4 & 78.00 \\
\hline HDAT & & (null) & & S111-RISER-4-5 & 102.00 \\
\hline HDAT & & (null) & & S111-RISER-4-6 & -126.00 \\
\hline HDAT & & (null) & & S111-RंISER-4-7 & 150.00 \\
\hline HDAT & & (null) & & S111-RISER-4-8 & 174.00 \\
\hline HDAT & & (null) & & S111-RISER-4-9 & 198.00 \\
\hline HDAT & & (null) & 1 & S112-RISER-4-1 & 6.00 \\
\hline HDAT & & (null) & & S112-RISER-4-10 & 222.00 \\
\hline HDAT & & (null) & & S112-RISER-4-11 & 246.00 \\
\hline HDAT & & (null) & & S112-RISER-4-12 & 270.00 \\
\hline HDAT & & - (null) & i & S112-RISER-4-13 & 318.00 \\
\hline HDAT & & (null) & $!$ & S112-RISER-4-14 & 366.00 \\
\hline HDAT & & (null) & $\vdots$ & S112-RISER-4-2 & 30.00 \\
\hline HDAT & & (null) & & S112-RISER-4-3 & 54.00 \\
\hline HDAT & & (null) & & S112-RISER-4-4 & 78.00 \\
\hline HDAT & & (null) & & S112-RISER-4-5 & 102.00 \\
\hline HDAT & & (null) & & S112-RISER-4-6 & 126.00 \\
\hline HDAT & & (null) & & S112-RISER-4-7 & 150.00 \\
\hline HDAT & & (null) & & S112-RISER-4-8 & 174.00 \\
\hline HDAT & & (null) & & S112-RISER-4-9 & 198.00 \\
\hline HDAT & & (nuil) & & SX101-RISER-15-1 & 20.52 \\
\hline HDAT & & (null) & & SX101-RISER-15-2 & 44.04 \\
\hline HDÁT & & (null) & & SX101-RISER-15-3 & 68.04 \\
\hline HDAT & & (nuil) & & SX101-RISER-15-4 & 92.04 \\
\hline HDAT & & (nuli) & & SX101-RISER-15-5 & 116.04 \\
\hline HDAT & & (null) & & SX101-RISER-15-6 & 383.04 \\
\hline HDAT & & (null) & & SX102-RISER-16-1 & 17.88 \\
\hline HDAT & & (null) & & SX102-RISER-16-2 & 41.40 \\
\hline HDAT & & (null) & & SX102-RISER-16-3 & 65.40 \\
\hline HDAT & & (null) & & SX102-RISER-16-4 & 89.40 \\
\hline HDAT & & (null) & & SX102-RISER-16-5 & 113.40 \\
\hline HDAT & & (null) & & SX102-RISER-16-6 & 377.40 \\
\hline HDAT & & (null) & & SX103-RISER-2-1 & 408 \\
\hline HDAT & & (null) & & SX.103-RISER-2.2 & 27.60 \\
\hline HDAT & & (null) & & S׳.103-RISER-2-3 & 51.60 \\
\hline HDAT & & (nul1) & & $S \times 103-R I S E R-2-4$ & 75.60 \\
\hline HDAT & & (nuil) & & Sx103-RISER-2-5 & 99.60 \\
\hline HDAT & & (null) & & $3 \times 103-R I S E R-2-6$ & 366.60 \\
\hline
\end{tabular}


HNF-3776 REV. O PG. 55 OF 95

Surface Level (Inches)

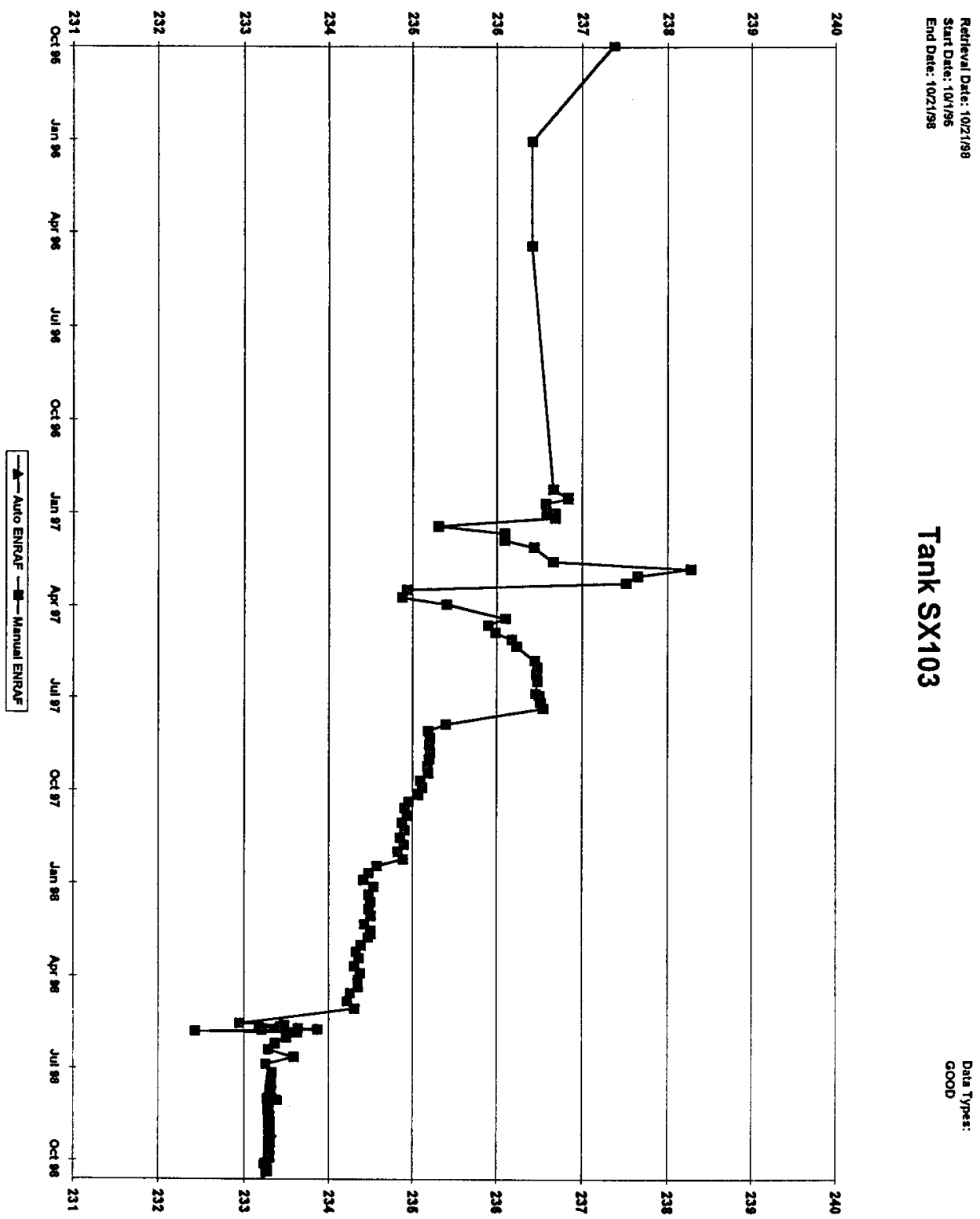


HNF-3776 REV. O PG. 56 OF 95

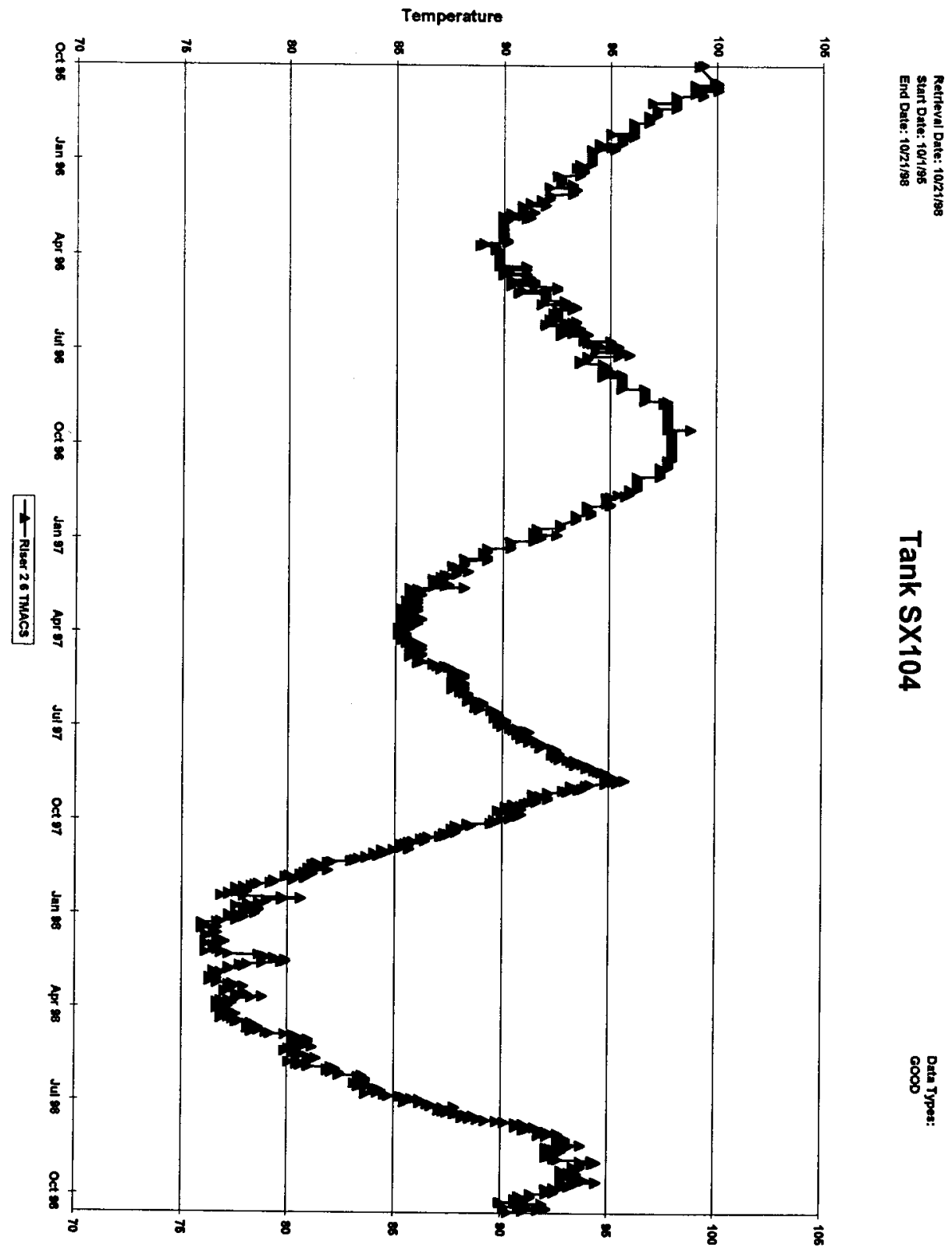


HNF-3776 REV. O PG. 57 OF 95

Surface Level (Inches)

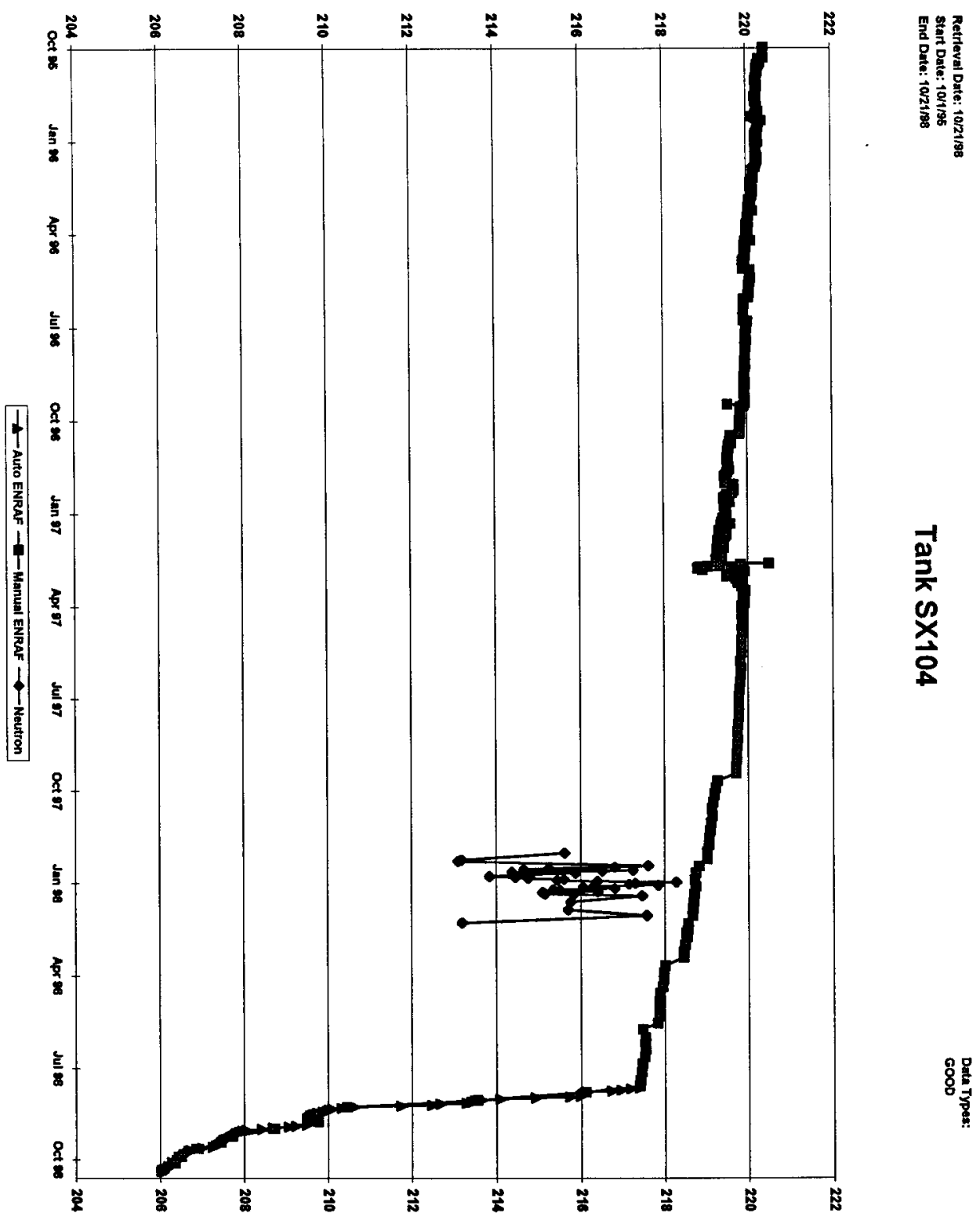


Sheet 1

\begin{tabular}{|c|c|c|c|}
\hline HDAT & (nuil) & SX104-RISER-2-1 & 4.08 \\
\hline HDAT & (null) & SX104-RISER-2-2 & 27.60 \\
\hline HDAT & (null) & SX104-RISER-2-3 & 51.60 \\
\hline HDAT & (null) & SX104-RISER-2-4 & 75.60 \\
\hline HDAT & (null) & SX104-RISER-2-5 & 99.60 \\
\hline HDAT & (null) & SX104-RISER-2-6 & 366.60 \\
\hline HDAT & (null) & SX105-RISER-2-1 & 4.00 \\
\hline HDAT & (null) & SX105-RISER-2-2 & 28.00 \\
\hline HDAT & (null) & SX105-RISER-2-3 & 52.00 \\
\hline HDAT & (null) & SX105-RISER-2-4 & 76.00 \\
\hline HDAT & (null) & SX105-RISER-2-5 & 100.00 \\
\hline HDAT & (null) & SX105-RISER-2-6 & 367.00 \\
\hline HDAT & (null) & SX106-RISER-16-1 & 13.00 \\
\hline HDAT & (null) & SX106-RISER-16-2 & 37.00 \\
\hline HDAT & (nuli) & SX106-RISER-16-3 & 61.00 \\
\hline HDAT & (nuli) & SX106-RISER-16-4 & 85.00 \\
\hline HDAT & (null) & SX106-RISER-16-5 & 109.00 \\
\hline HDAT & (null) & SX106-RISER-16-6 & 191.00 \\
\hline HDAT & (null) & SX107-RISER-10-1 & 4.00 \\
\hline HDAT & (null) & SX107-RISER-10-2 & 12.00 \\
\hline HDAT & (null) & SX107-RISER-10-3 & 21.00 \\
\hline HDAT & (null) & SX107-RISER-10-4 & 29.00 \\
\hline HDAT & (null) & SX107-RISER-10-5 & 38.00 \\
\hline HDAT & (null) & SX107-RISER-10-6 & 46.00 \\
\hline HDAT & (null) & SX107-RISER-10-7 & 54.00 \\
\hline HDAT & (null) & SX107-RISER-10-8 & 62.00 \\
\hline HDAT & (null) & SX107-RISER-14-1 & 4.00 \\
\hline HDAT & (nuil) & SX107-RISER-14-2 & 12.00 \\
\hline HDAT & (null) & SX107-RISER-14-3 & 21.00 \\
\hline HDAT & (null) & SX107-RISER-14-4 & 29.00 \\
\hline HDAT & (null) & SX107-RISER-14-5 & 38.00 \\
\hline HDAT & (nuil) & SX107-RISER-14-6 & 46.00 \\
\hline HDAT & (null) & SX107-RISER-14-7 & 54.00 \\
\hline HDAT & (null) & SX107-RISER-14-8 & 62.00 \\
\hline HDAT & (null) & SX108-RISER-10-1. & 4.00 \\
\hline HDAT & (null) & SX108-RISER-10-2 & 21.00 \\
\hline HDAT & (null) & SX108-RISER-10-3 & 38.00 \\
\hline HDAT & (null) & SX108-RISER-10-4 & 54.00 \\
\hline HDAT & (null) & SX108-RISER-19-1 & 4.00 \\
\hline HDAT & (null) & SX108-RISER-19-2 & 21.00 \\
\hline HDAT & (null) & SX108-RISER-19-3 & 38.00 \\
\hline HDAT & (null) & SX108-RISER-19-4 & 54.00 \\
\hline HDAT & (null) & SX109-RISER-10-1 & 4.00 \\
\hline HDAT & (null) & SX109-RISER-10-2 & 12.00 \\
\hline HDAT & (null) & SX109-RISER-10-3 & 21.00 \\
\hline HDAT & (null) & SX109-RISER-10-4 & 29.00 \\
\hline HDAT & (null) & SX109-RISER-10-5 & 38.00 \\
\hline HDAT & (nuli) & SX109-RISER-10-6 & 46.00 \\
\hline HDAT & (nuli) & SX109-RISER-10-7 & 54.00 \\
\hline HDAT & (null) & SX109-RISER-10-8 & 62.00 \\
\hline HDAT & (null) & SX109-RISER-19-1 & 4.00 \\
\hline
\end{tabular}


HNF-3776 REV. O PG. 59 OF 95

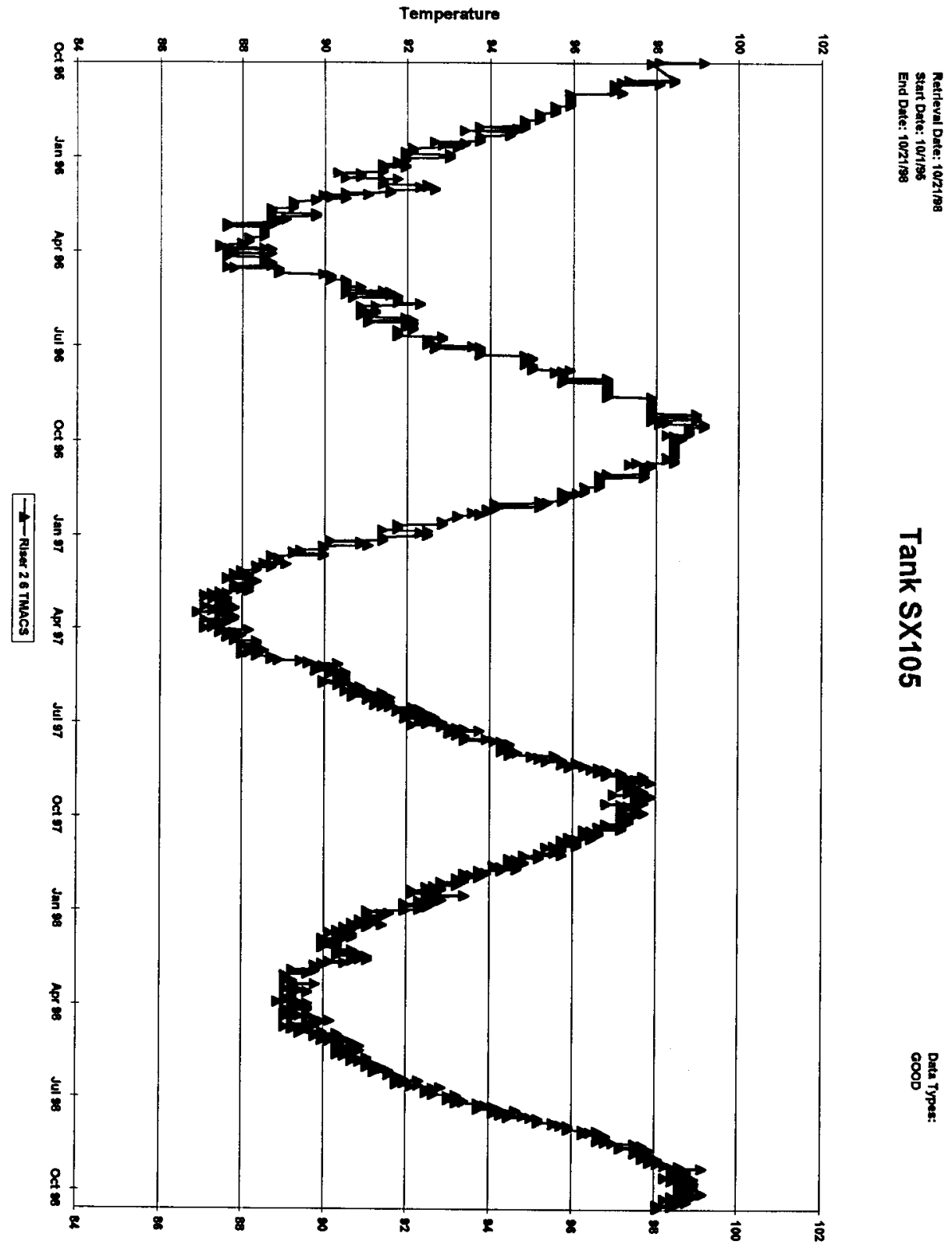


HNF-3776 REV. O PG. 60 OF 95

Surface Level (inches)

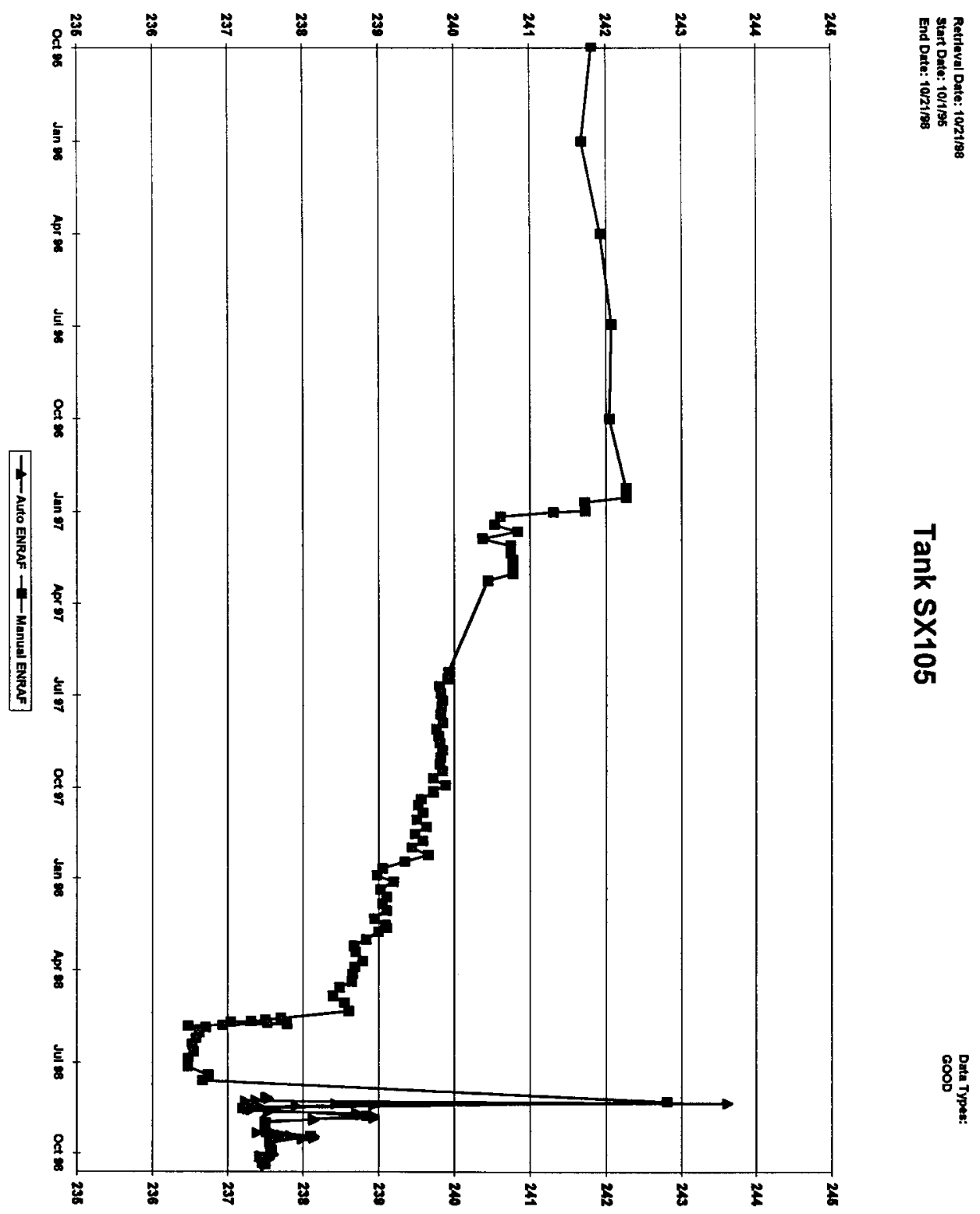


Sheet1

\begin{tabular}{|c|c|c|c|c|}
\hline HDAT & & (null) & SX104-RISER-2-1 & 4.08 \\
\hline HDAT & & (null) & SX104-RISER-2-2 & 27.60 \\
\hline HDAT & & (null) & SX104-RISER-2-3 & 51.60 \\
\hline HDAT & & (null) & SX104-RISER-2-4 & 75.60 \\
\hline HDAT & & (null) & SX104-RISER-2-5 & 99.60 \\
\hline HDAT & & (null) & SX104-RISER-2-6 & 366.60 \\
\hline HDAT & & (null) & SX105-RISER-2-1 & 4.00 \\
\hline HDAT & & (nu!l) & SX105-RISER-2-2 & 28.00 \\
\hline HDAT & & (null) & SX105-RISER-2-3 & 52.00 \\
\hline HDAT & & (null) & SX105-RISER-2-4 & 76.00 \\
\hline HDAT & & (null) & SX105-RISER-2-5 & 100.00 \\
\hline HDAT & & (null) & SX105-RISER-2-6 & 367.00 \\
\hline HDAT & & (null) & SX106-RISER-16-1 & 13.00 \\
\hline HDAT & & (null) & SX106-RISER-16-2 & 37.00 \\
\hline HDAT & & (null) & SX106-RISER-16-3 & 61.00 \\
\hline HDAT & & (null) & SX106-RISER-16-4 & 85.00 \\
\hline HDAT & & (null) & SX106-RISER-16-5 & 109.00 \\
\hline HDAT & & (null) & SX106-RISER-16-6 & 191.00 \\
\hline HDAT & & (null) & SX107-RISER-10-1 & 4.00 \\
\hline HDAT & & (null) & SX107-RISER-10-2 & 12.00 \\
\hline HDAT & & (null) & SX107-RISER-10-3 & 21.00 \\
\hline HDAT & & (null) & SX107-RISER-10-4 & 29.00 \\
\hline HDAT & & (null) & SX107-RISER-10-5 & 38.00 \\
\hline HDAT & & (null) & SX107-RISER-10-6 & 46.00 \\
\hline HDAT & & (null) & SX107-RISER-10-7 & 54.00 \\
\hline HDAT & & (null) & SX107-RISER-10-8 & 62.00 \\
\hline HDAT & & (null) & SX107-RISER-14-1 & 4.00 \\
\hline HDAT & & (null) & SX107-RISER-14-2 & 12.00 \\
\hline HDAT & & (null) & SX107-RISER-14-3 & 21.00 \\
\hline HDAT & & (null) & SX107-RISER-14-4 & 29.00 \\
\hline HDAT & & (null) & SX107-RISER-14-5 & 38.00 \\
\hline HDAT & & (null) & SX107-RISER-14-6 & 46.00 \\
\hline HDAT & - & (null) & SX107-RISER-14-7 & 54.00 \\
\hline HDÁT & & (null) & SX107-RISER-14-8 & 62.00 \\
\hline HDAT & & (nuil) & SX108-RISER-10-1 & 4.00 \\
\hline HDAT & & (nuld) & SX108-RISER-10-2 & 21.00 \\
\hline HDAT & & (null) & SX108-RISER-10-3 & 38.00 \\
\hline HDAT & & (null) & SX108-RISER-10-4 & 54.00 \\
\hline HDAT & & (nuil) & SX108-RISER-19-1 & 4.00 \\
\hline HDAT & & (null) & S×108-RISER-19-2 & 21.00 \\
\hline HDAT & & (nuli) & SX108-RISER-19-3 & 38.00 \\
\hline HDAT & & (null) & SX108-RISER-19-4 & 54.00 \\
\hline HDAT & & (null) & SX109-RISER-10-1 & 4.00 \\
\hline HDAT & & (null) & SX109-RISER-10-2 & 12.00 \\
\hline HDAT & & (null) & SX109-RISER-10-3 & 21.00 \\
\hline HDAT & & (null) & SX109-RISER-10-4 & 29.00 \\
\hline HDAT & & (null) & SX109-RISER-10-5 & 38.00 \\
\hline HDAT & & $(\mathrm{null})$ & S×108-RISER-10-6 & 46.00 \\
\hline HDAT & & (null) & S×109-RISER-10-7 & 54,00 \\
\hline HDAT & & (null) & SX109-RISER-10-8 & 62.00 \\
\hline HDAT & & (null) & SX109-RISER-19-1 & 4.00 \\
\hline
\end{tabular}




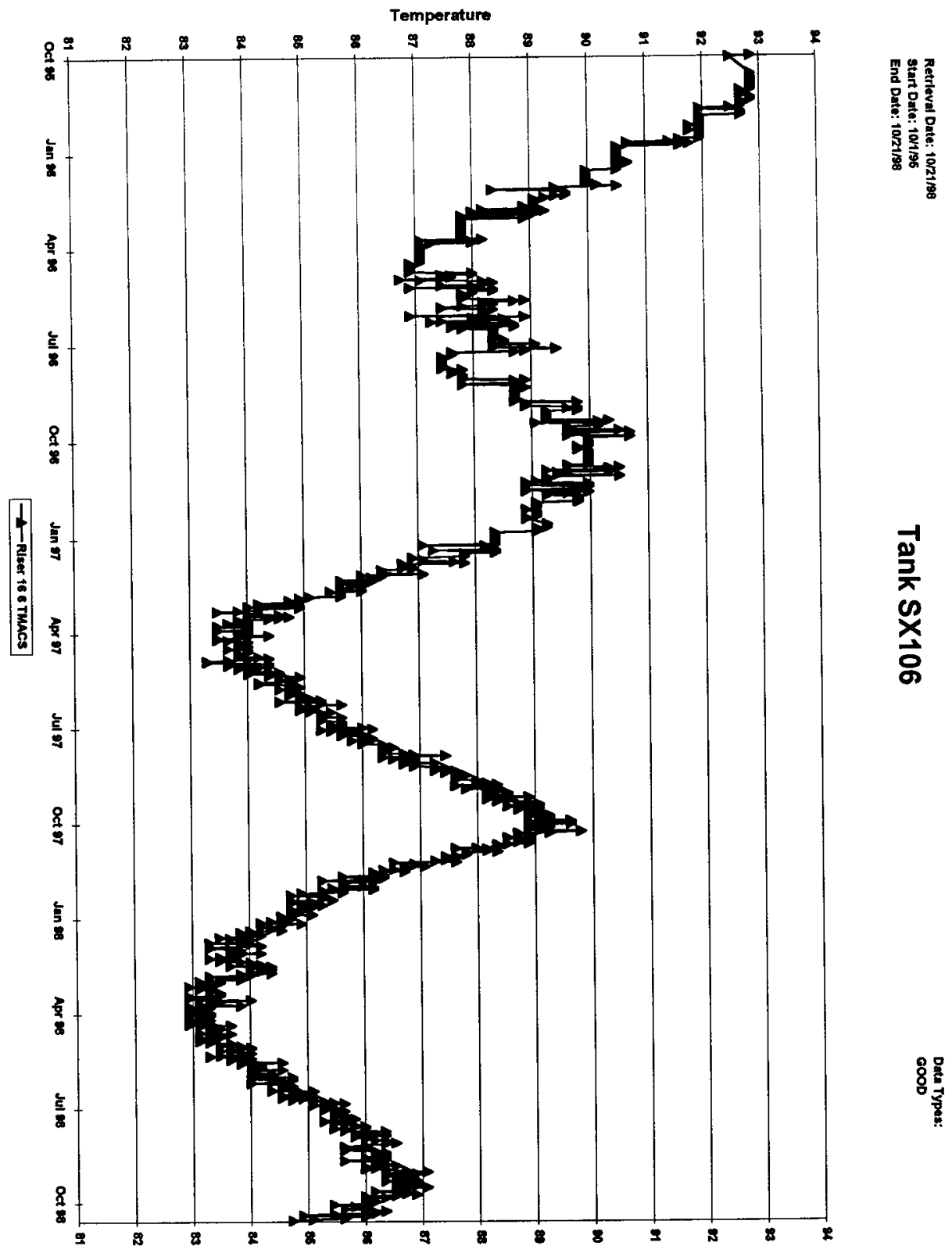


Surface Level (Inches)

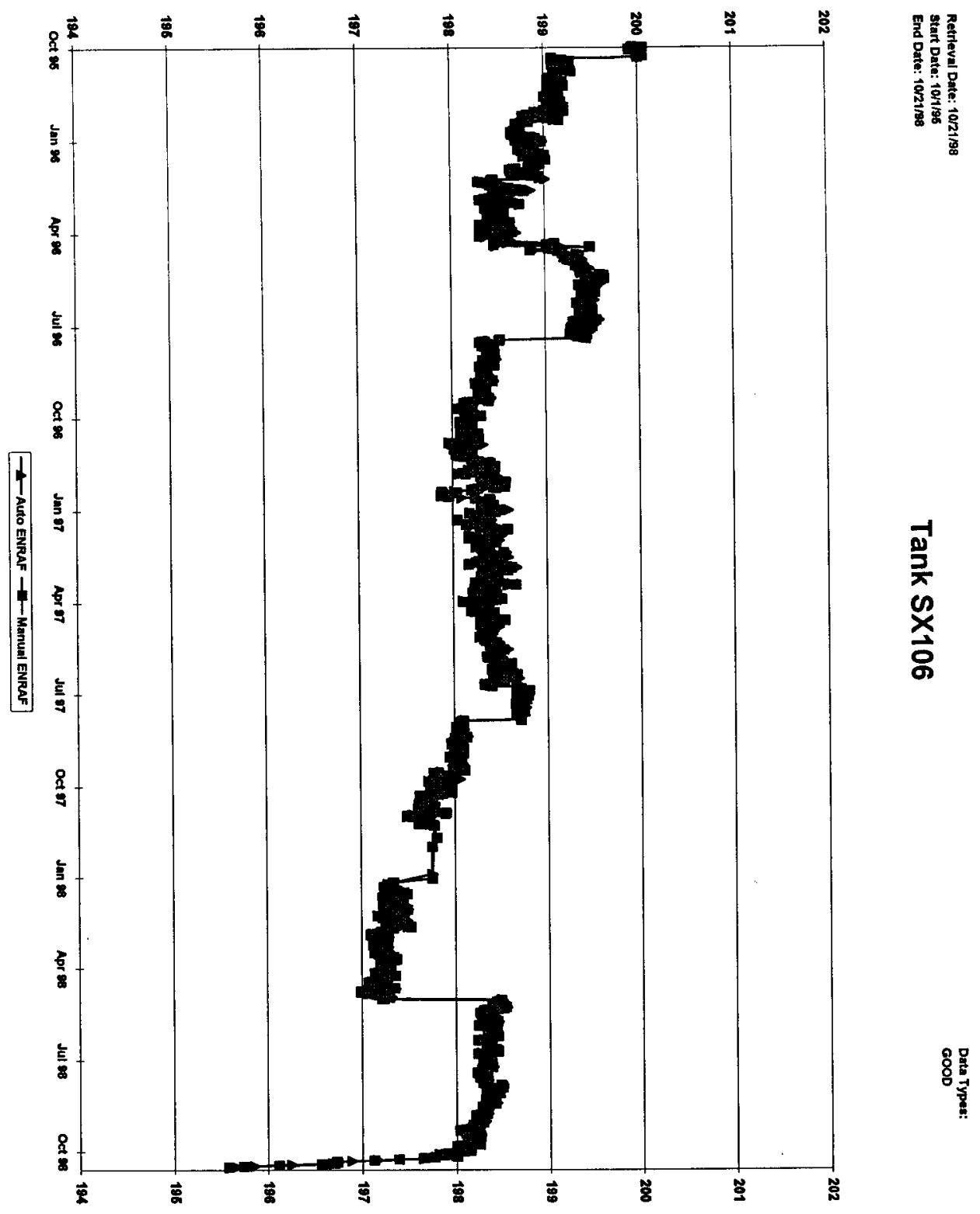


Sheet1

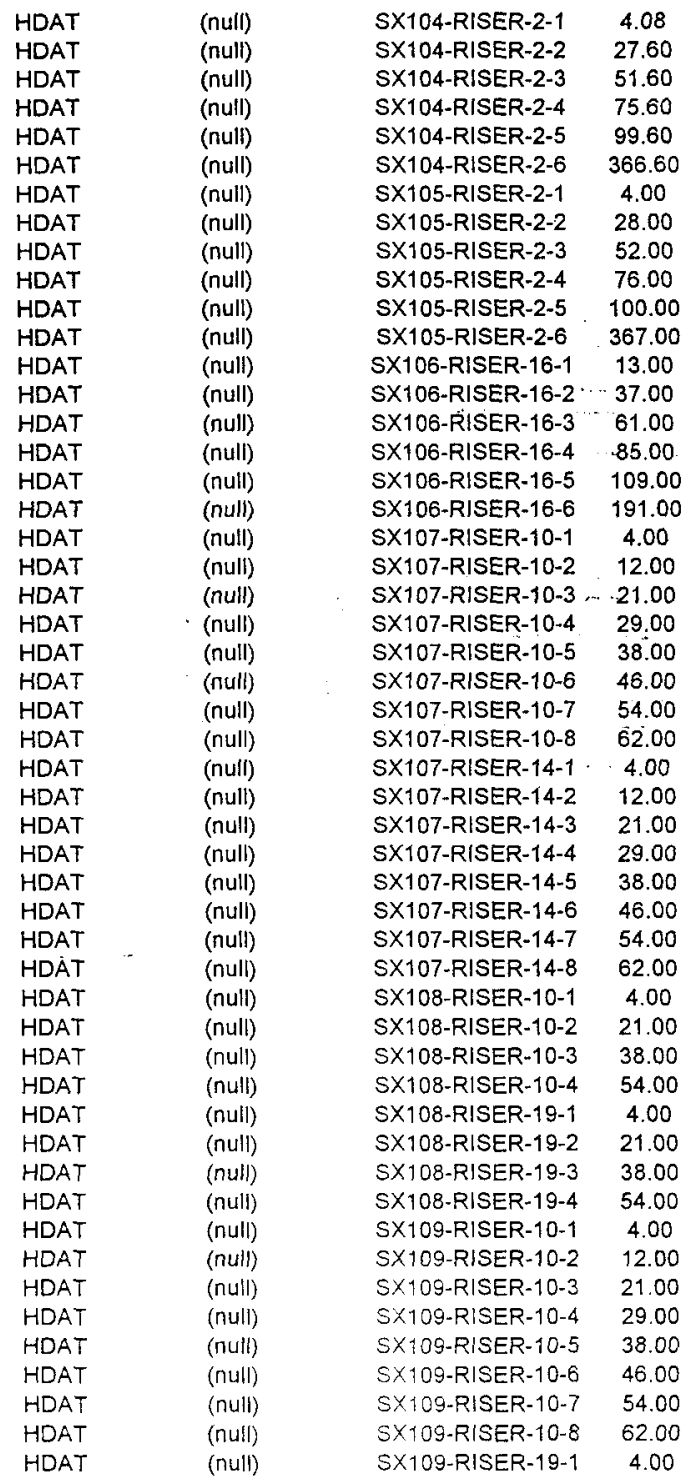




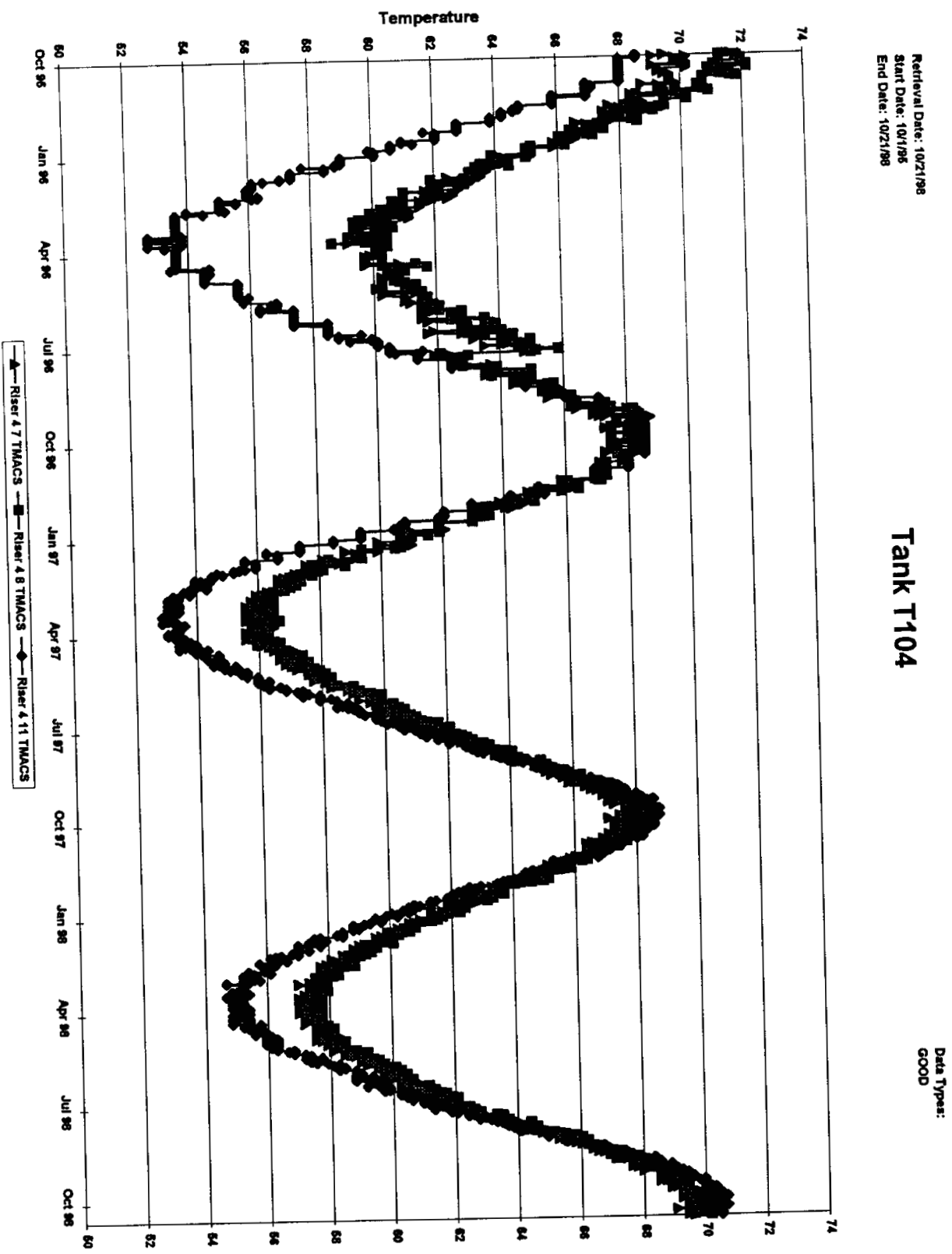




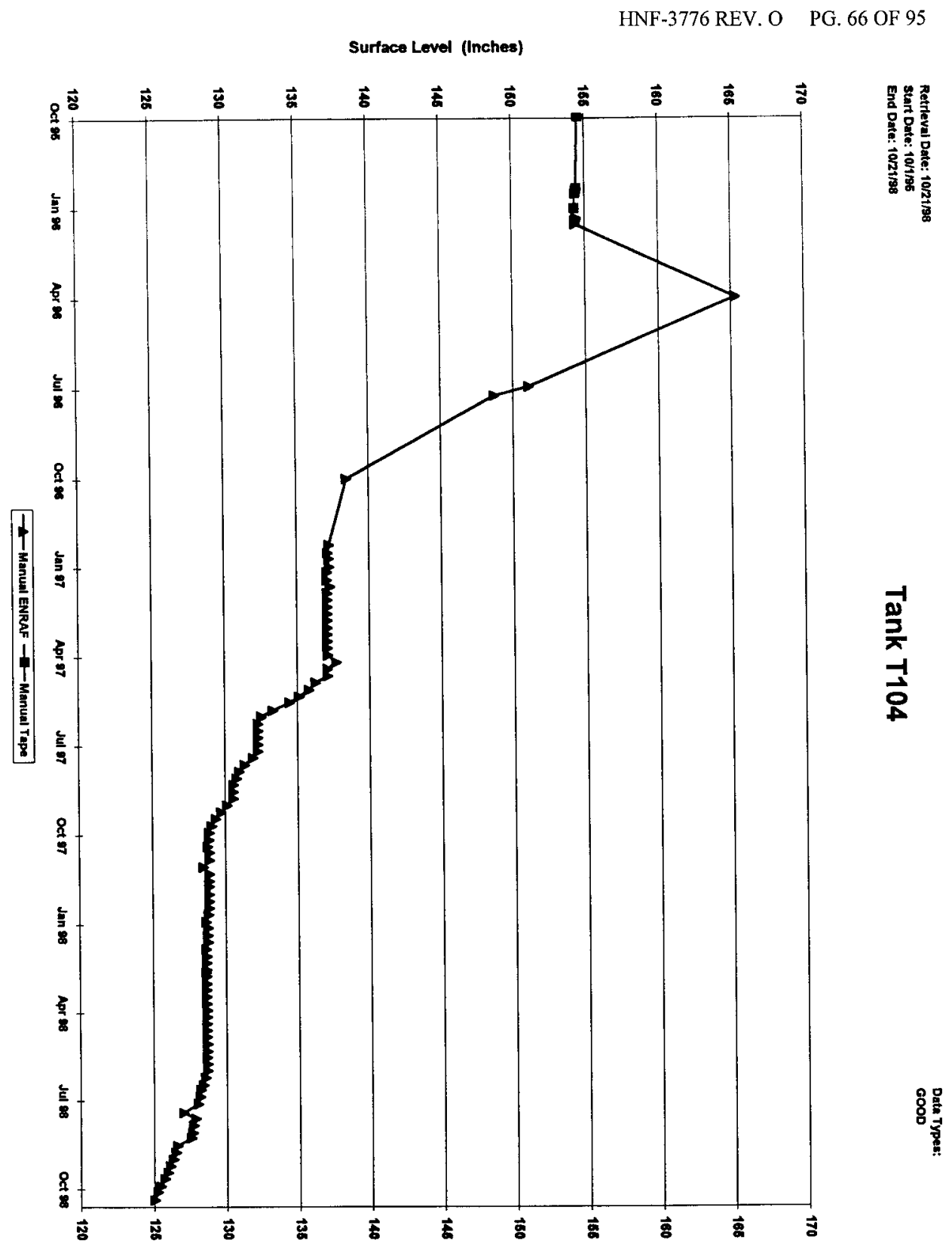




\begin{tabular}{|c|c|c|c|}
\hline IACS & SY103-TI-R17B-01 & SY-103-RISER-17B-1 & - \\
\hline MACS & SY103-TI-R17B-02 & SY-103-RISER-17B-2 & 16.00 \\
\hline MACS & SY103-TI-R17B-03 & SY-103-RISER-17B-3 & \\
\hline MACS & SY103-TI-R17B-04 & SY-103-RISER-17B-4 & 2.00 \\
\hline MACS & SY103-TI-R17B-05 & SY-103-RISER-17B-5 & 6.00 \\
\hline MACS & SY103-TI-R17B-06 & SY-103-RISER-17B-6 & 100.00 \\
\hline MACS & SY103-TI-R17B-07 & SY-103-RISER-17B-7 & 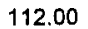 \\
\hline & SY103-TI-R17B-08 & SY-103-RISER-17B-8 & 24.00 \\
\hline TMACS & SY103-TI-R17B-09 & ISER-17B-9 & 8.00 \\
\hline TMACS & SY103-TI-R17B-10 & SY-103 & 172.00 \\
\hline TMACS & SY103-TI-R17B-11 & SY-103-RISER-17B-11 & 196.00 \\
\hline TMACS & SY103-TI-R17B-12 & SY-103-RISER-17B-12 & 208.00 \\
\hline TMACS & SY103-TI-R17B-13 & SY-103-RISER-17B-13 & 220.00 \\
\hline TMACS & 217B-14 & SY-103-RISER-17B-14 & 232.00 \\
\hline TMACS & 17B-15 & ISER-17B-15 & 244.00 \\
\hline TMACS & SY103-Tl- & SY-103-RISER-17B-16 & 268.00 \\
\hline TMACS & $17 \mathrm{~B}-17$ & ISER-17B-17 & 292.00 \\
\hline TMACS & 17B-18 & $78-18$ & 326.00 \\
\hline TMACS & $78-19$ & 7B-19 & 340.00 \\
\hline TMACS & $7 \mathrm{~B}-20$ & 7B-20 & 364.00 \\
\hline TMACS & $B-21$ & $\mathrm{~B}-21$ & 392.00 \\
\hline TMACS & $7 \mathrm{~B}-22$ & $7 B-22$ & 402.00 \\
\hline TMACS & U-104 & 104-1 & (null) \\
\hline TMACS & $\mathrm{J}-107$ & $07-1$ & (null) \\
\hline TMACS & $U-112$ & $12-1$ & (null) \\
\hline TMACS & $U-115$ & $15-1$ & (null) \\
\hline TMACS & U-92 & $92-1$ & (null) \\
\hline TMACS & U-95 & $95-1$ & (nuli) \\
\hline TMACS & $3-01$ & -1 & 14.40 \\
\hline TMACS & 3-02 & -2 & 38.40 \\
\hline TMACS & $8-03$ & ISE & 62.40 \\
\hline TMACS & 8-08 & ISEI & 182.40 \\
\hline TMACS & 3-11 & SEF & 302.40 \\
\hline TMACS & 1 & ISE & 14.00 \\
\hline TMACS & 02 & -2 & 38.00 \\
\hline TMACS & & -7 & 158.00 \\
\hline TMACS & $3-11$ & 11 & 302.00 \\
\hline TMACS & & SE & 14.00 \\
\hline TMACS & $04-02$ & ISEF & 38.00 \\
\hline TMACS & T104-T & ISEF & 62.00 \\
\hline TMACS & 04-04 & ISEF & 86.00 \\
\hline TMACS & 4-05 & ISE & 110.00 \\
\hline TMACS & & ISEF & 134.00 \\
\hline TMACS & $4-07$ & ISEF & 158.00 \\
\hline TMACS & $4-08$ & ISER-4-8 & 182.00 \\
\hline & & शISER-4-11 & 302.00 \\
\hline TMACS & T106-TI-R008-01 & T106-RISER-8-1 & 14.00 \\
\hline TMACS & $08-02$ & RISER-8-2 & 38.00 \\
\hline TMACS & T106-TI- & T106-RISER-8-7 & 158.00 \\
\hline TMACS & T106-TI- & T106-RISER-8-11 & 302.00 \\
\hline TMACS & T107-TI-R004-01 & T107-RISER-4-1 & 14.40 \\
\hline
\end{tabular}




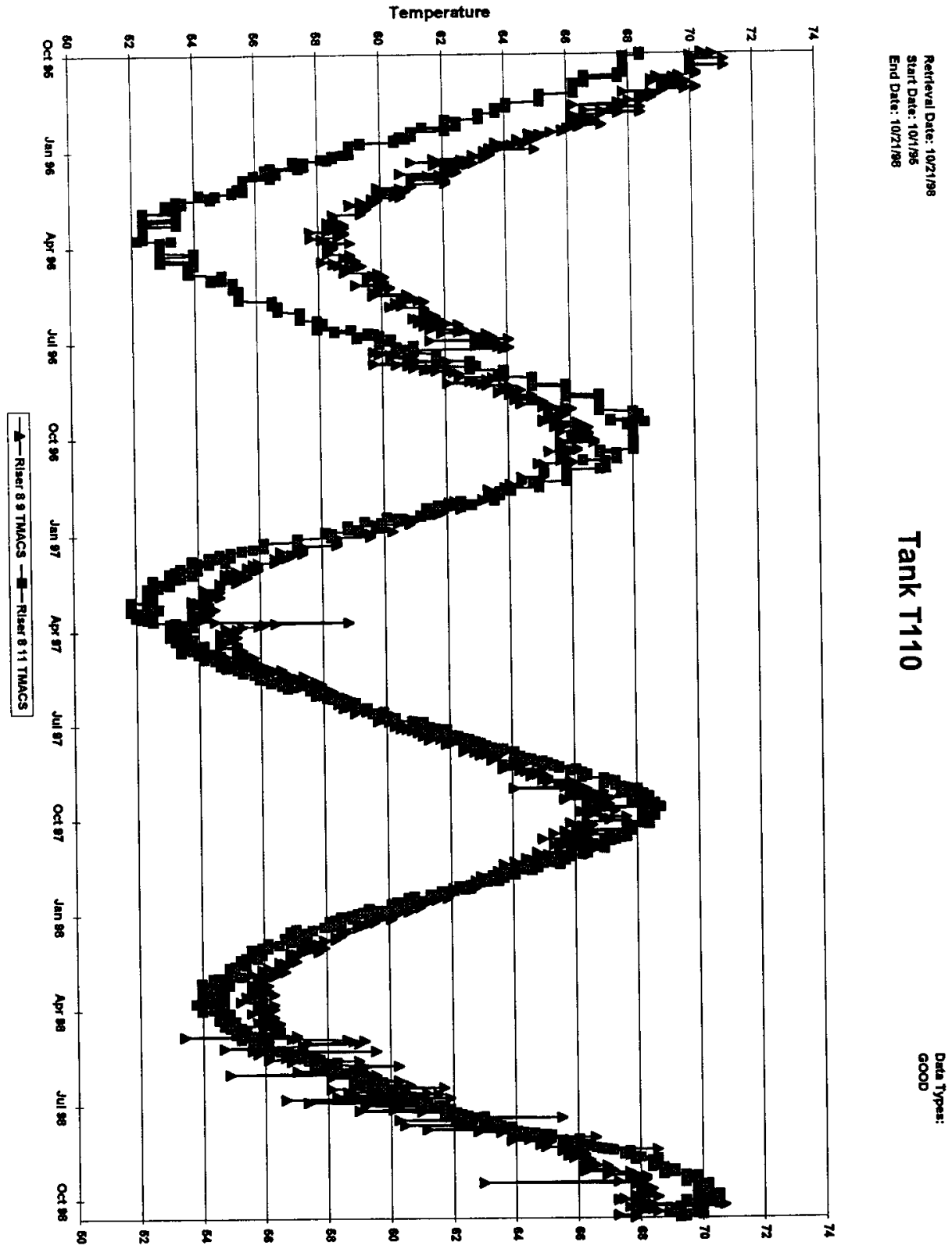




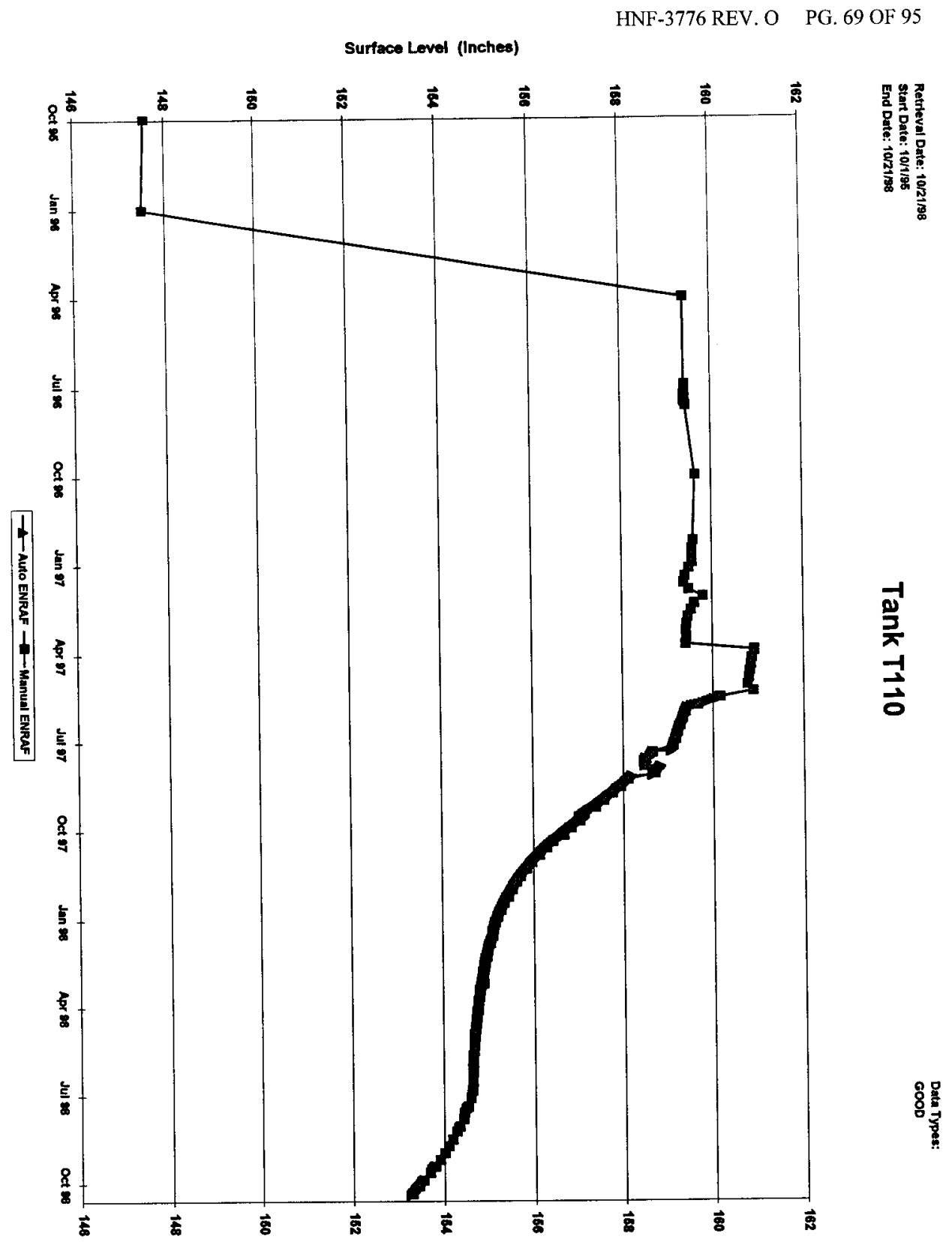


Sheet1

\begin{tabular}{|c|c|c|c|}
\hline TMACS & T107-TI-R004-02 & T107-RISER-4-2 & 38.40 \\
\hline TMACS & T107-TI-R004-03 & T107-RISER-4-3 & 62.40 \\
\hline TMACS & T107-TI-R004-04 & T107-RISER-4-4 & 86.40 \\
\hline TMACS & T107-TI-R004-08 & T107-RISER-4-8 & 182.40 \\
\hline TMACS & T107-TI-R004-11 & T107-RISER-4-11 & 302.40 \\
\hline TMACS & T107-TI-R005-01 & T107-RISER-5-1 & 14.00 \\
\hline TMACS & T107-Tl-R005-02 & T107-RISER-5-2 & 18.00 \\
\hline TMACS & T107-TI-R005-03 & T107-RISER-5-3 & 24.00 \\
\hline TMACS & T107-TI-R005-04 & T107-RISER-5-4 & 30.00 \\
\hline TMACS & T107-TI-R005-05 & T107-RISER-5-5 & 42.00 \\
\hline TMACS & T107-TI-R005-06 & T107-RISER-5-6 & 54.00 \\
\hline TMACS & T107-TL-R005-07 & T107-RISER-5-7 & 66.00 \\
\hline TMACS & T107-TI-R005-08 & T107-RISER-5-8 & 262.00 \\
\hline TMACS & T108-TI-R004-01 & T108-RISER-4-1 & 14.00 \\
\hline TMACS & T108-TI-R004-02 & T108-RISER-4-2 & 38.00 \\
\hline TMACS & T108-TI-R004-07 & T108-RISER-4-7 & 158.00 \\
\hline TMACS & T108-TI-R004-11 & T108-RISER-4-11 & 302.00 \\
\hline TMACS & T109-TI-R008-01 & T109-RISER-8-1 & 14.00 \\
\hline TMACS & T109-TI-R008-02 & T109-RISER-8-2 & 38.00 \\
\hline TMACS & T109-TI-R008-03 & T109-RISER-8-3 & 62.00 \\
\hline TMACS & T109-TI-R008-07 & T109-RISER-8-7 & 158.00 \\
\hline TMACS & T109-TI-R008-11 & T109-RISER-8-11 & 302.00 \\
\hline TMACS & T110-TI-R008-01 & T110-RISER-8-1 & 14.00 \\
\hline TMACS & T110-TI-R008-02 & T110-RISER-8-2 & 38.00 \\
\hline TMACS & T110-TI-R008-03 & T110-RISER-8-3 & 62.00 \\
\hline TMACS & T110-TI-R008-04 & T110-RISER-8-4 & 86.00 \\
\hline TMACS & T110-TI-R008-05 & T110-RISER-8-5 & 110.00 \\
\hline TMACS & T110-T1-R008-06 & T110-RISER-8-6 & 134.00 \\
\hline TMACS & T110-TI-R008-07 & T110-RISER-8-7 & 158.00 \\
\hline TMACS & T110-TI-R008-09 & T110-RISER-8-9 & 206.00 \\
\hline TMACS & T†10-Tl-R008-11 & T110-RISER-8-11 & 302.00 \\
\hline TMACS & T111-TI-R005-01 & T111-RISER-5-1 & 14.00 \\
\hline TMACS & T111-Tl-R005-02 & T111-RISER-5-2 & 38.00 \\
\hline TMACS & T111-TI-R005-03 & T111-RISER-5-3 & 62.00 \\
\hline TMACS & T111-T1-R005-04 & T111-RISER-5-4 & 86.00 \\
\hline TMACS & T111-TI-R005-05 & T111-RISER-5-5 & 110.00 \\
\hline TMACS & T111-TI-R005-06 & T111-RISER-5-6 & 134.00 \\
\hline TMACS & T111-TI-R005-07 & T111-RISER-5-7 & 158.00 \\
\hline TMACS & T111-TI-R005-08 & T111-RISER-5-8 & 182.00 \\
\hline TMACS & T111-TI-R005-11 & T111-RISER-5-11 & 302.00 \\
\hline TMACS & T112-TI-R008-01 & T112-RISER-8-1 & 14.00 \\
\hline TMACS & T112-TI-R008-02 & T112-RISER-8-2 & 38.00 \\
\hline TMACS & T112-TI-R008-03 & T112-RISER-8-3 & 62.00 \\
\hline TMACS & T112-TI-R008-07 & T112-RISER-8-7 & 158.00 \\
\hline TMACS & T112-TI-R008-11 & T112-RISER-8-11 & 302.00 \\
\hline TMACS & T201-TI-R005-01 & T201-RISER-5-1 & 14.00 \\
\hline TMACS & T201-TI-R005-02 & T201-RISER-5-2 & 38.00 \\
\hline TMACS & T201-TI-R005-03 & T201-RISER-5-3 & 62.00 \\
\hline TMACS & T201-TI-R005-04 & T201-RISER-5-4 & 86.00 \\
\hline TMACS & T201-TI-R005-05 & T201-RISER-5-5 & 110.00 \\
\hline TMACS & T201-TI-R005-06 & T201-RISER-5-6 & 134.00 \\
\hline
\end{tabular}




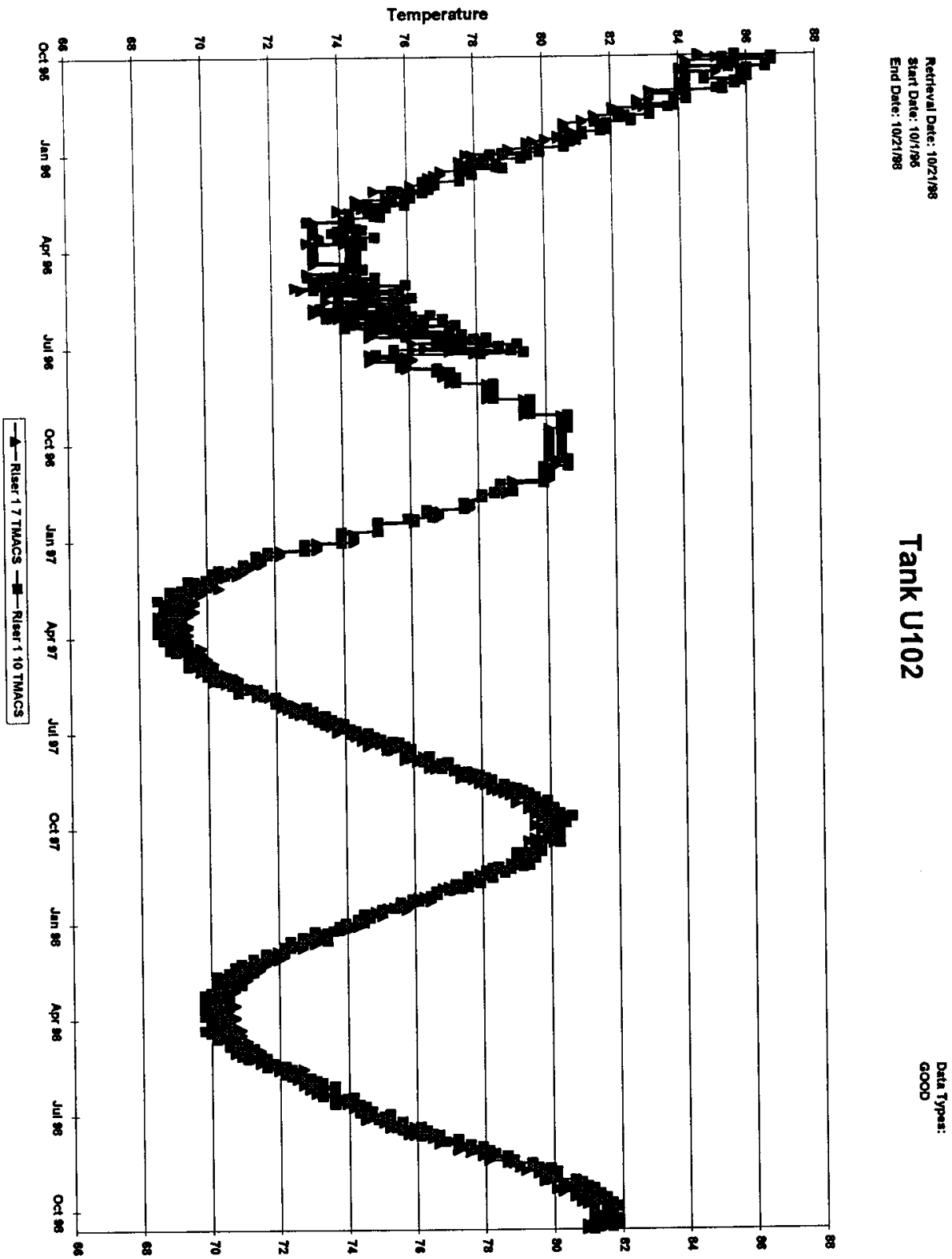


HNF-3776 REV. O PG. 72 OF 95

Surface Level (Inches)

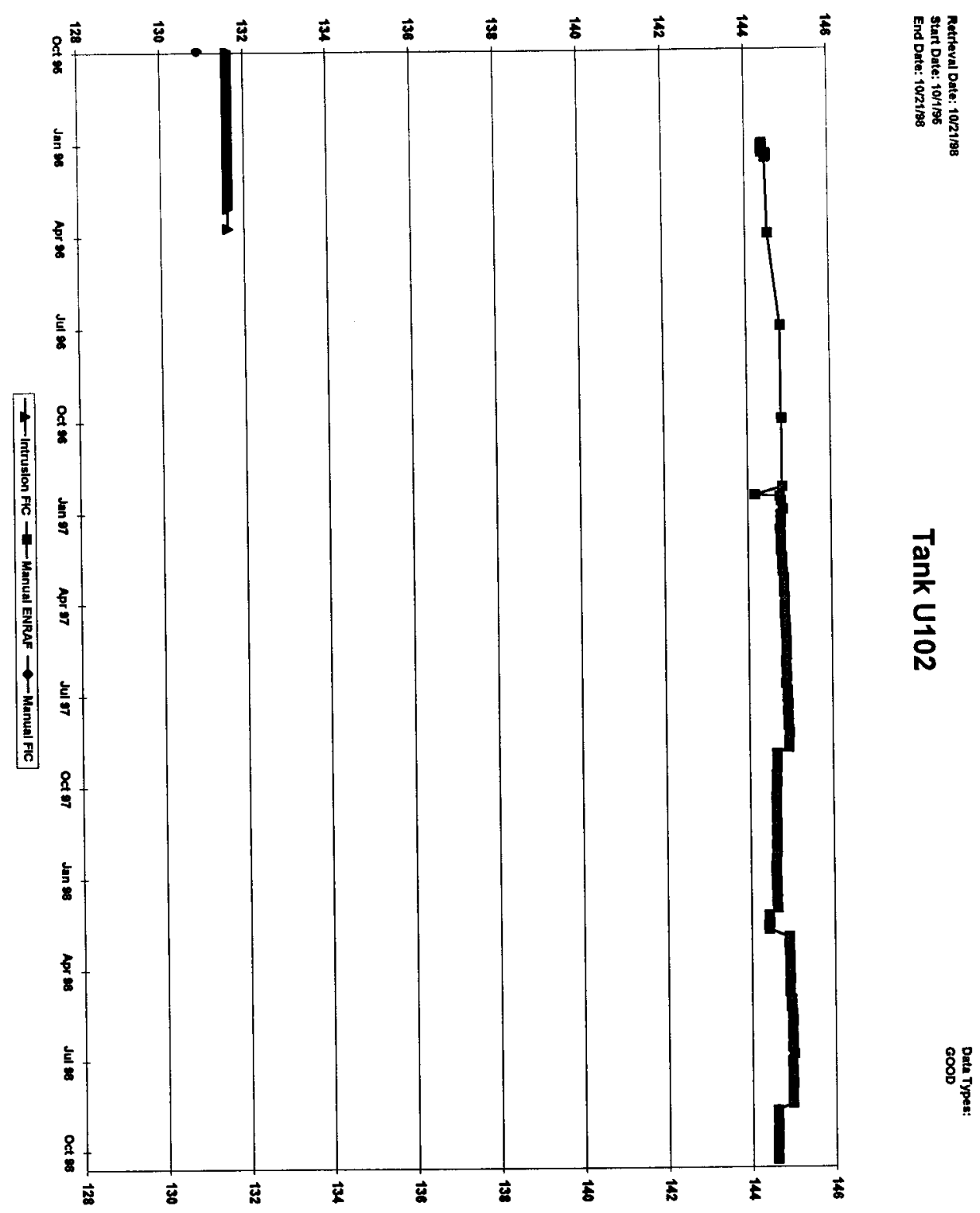


Sheet1

\begin{tabular}{|c|c|c|c|}
\hline HDAT & (null) & U102-RISER-1-10 & 220.00 \\
\hline HDAT & (null) & U102-RISER-1-11 & 244.00 \\
\hline HDAT & (null) & U102-RISER-1-12 & 268.00 \\
\hline HDAT & (null) & U102-RISER-1-13 & 316.00 \\
\hline HDAT & (null) & U102-RISER-1-14 & 364.00 \\
\hline HDAT & (null) & U102-RISER-1-2 & 28.00 \\
\hline HDAT & (null) & U102-RISER-1-3 & 52.00 \\
\hline HDAT & (null) & U102-RISER-1-4 & 76.00 \\
\hline HDAT & (null) & U102-RISER-1-5 & 100.00 \\
\hline HDAT & (null) & U102-RISER-1-6 & 124.00 \\
\hline HDAT & (null) & U102-RISER-1-7 & 148.00 \\
\hline HDAT & (null) & U102-RISER-1-8 & 172.00 \\
\hline HDAT & (null) & U102-RISER-1-9 & 196.00 \\
\hline HDAT & (null) & U103-RISER-1-1 & 21.36 \\
\hline HDAT & (null) & U103-RISER-1-10 & 261.36 \\
\hline HDAT & (null) & U103-RISER-1-11 & 309.36 \\
\hline HDAT & (null) & U103-RISER-1-2 & 45.36 \\
\hline HDAT & (null) & U103-RISER-1-3 & 69.36 \\
\hline HDAT & (null) & U103-RISER-1-4 & 93.36 \\
\hline HDAT & (null) & U103-RISER-1-5 & 117.36 \\
\hline HDAT & (null) & Ut03-RISER-1-6 & 141.36 \\
\hline HDAT & (null) & U103-RISER-1-7 & 165.36 \\
\hline HDAT & (null) & U103-RISER-1-8 & 189.36 \\
\hline HDAT & (null) & U103-RISER-1-9 & 213.36 \\
\hline HDAT & (null) & U105-RISER-1-1 & 21.36 \\
\hline HDAT & (null) & U105-RISER-1-10 & 261.36 \\
\hline HDAT & (null) & U105-RISER-1-†1 & 309.36 \\
\hline HDAT & (nuil) & U105-RISER-1-2 & 45.36 \\
\hline HDAT & (null) & U105-RISER-1-3 & 69.36 \\
\hline HDAT & (null) & U105-RISER-1-4 & 93.36 \\
\hline HDAT & (null) & U105-RISER-1-5 & 117.36 \\
\hline HDAT & (null) & U105-RISER-1-6 & 141.36 \\
\hline HDAT & (null) & U105-RISER-1-7 & 165.36 \\
\hline HDAT & (null) & U105-RISER-1-8 & 189.36 \\
\hline HDAT & (null) & U105-RISER-1-9 & 213.36 \\
\hline HDAT & (null) & U106-RISER-1-1 & 20.16 \\
\hline HDAT & (null) & U106-RISER-1-10 & 260.16 \\
\hline HDAT & (null) & U106-RISER-1-11 & 308.16 \\
\hline HDAT & (null) & U106-RISER-1-2 & 44.16 \\
\hline HDAT & (null) & U106-RISER-1-3 & 68.16 \\
\hline HDAT & (null) & U106-RISER-1-4 & 92.16 \\
\hline HDAT & (null) & U106-RISER-1-5 & 116.16 \\
\hline HDAT & (nuil) & U106-RISER-1-6 & 140.16 \\
\hline HDAT & (nuil) & U106-RISER-1-7 & 164.16 \\
\hline HDAT & (nuil) & U106-RISER-1-8 & 188.16 \\
\hline HDAT & (null) & U106-RISER-1-9 & 212.16 \\
\hline HDAT & (nuil) & U107-RISER-1-1 & 4.00 \\
\hline HDAT & (null) & U107-RISER-1-10 & 220.00 \\
\hline HDAT & (nuli) & U107-RISER-1-11 & 244.00 \\
\hline HDAT & (null) & U107-RISER-1-12 & 268.00 \\
\hline HDAT & (null) & U107-RISER-1-13 & 316.00 \\
\hline
\end{tabular}


HNF-3776 REV. O PG. 74 OF 95

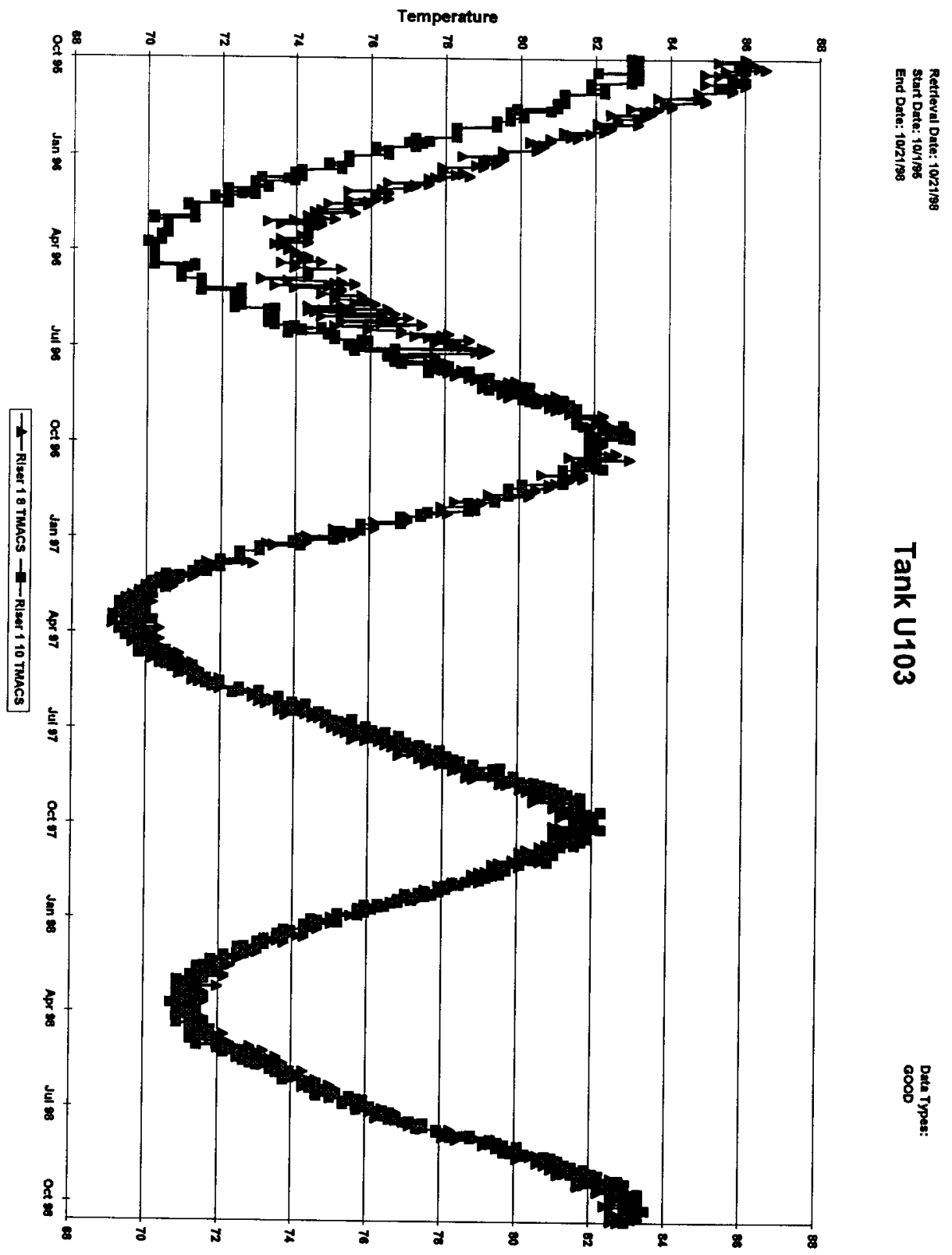




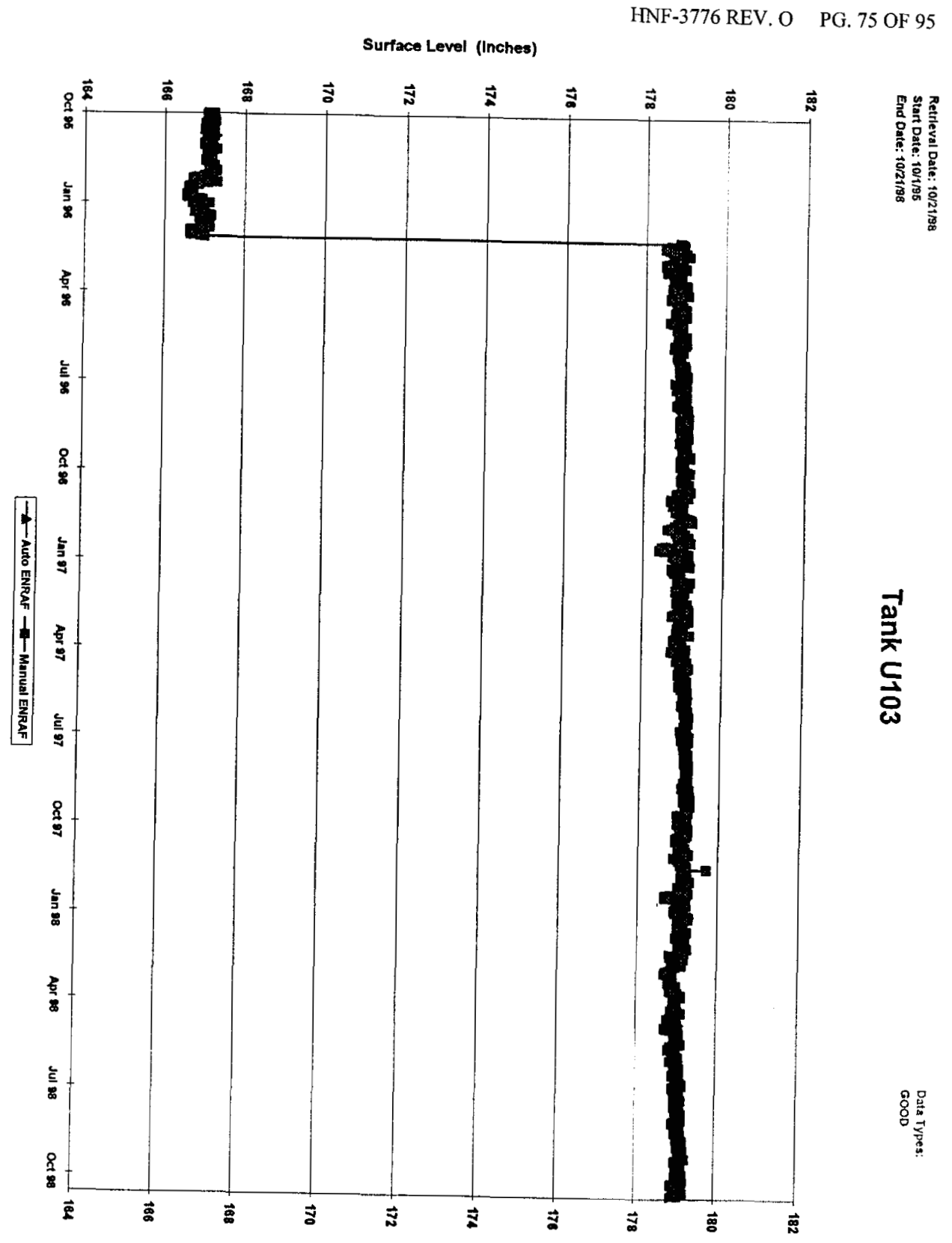




\begin{tabular}{|c|c|c|c|}
\hline HDAT & (null) & U102-RISER-1-10 & 220.00 \\
\hline HDAT & (null) & U102-RISER-1-11 & 244.00 \\
\hline HDAT & (null) & U102-RISER-1-12 & 268.00 \\
\hline HDAT & (null) & U102-RISER-1-13 & 316.00 \\
\hline HDAT & (null) & U102-RISER-1-14 & 364.00 \\
\hline HDAT & (null) & U102-RISER-1-2 & 28.00 \\
\hline HDAT & (null) & U102-RISER-1-3 & 52.00 \\
\hline HDAT & (null) & U102-RISER-1-4 & 76.00 \\
\hline HDAT & (nu!l) & U102-RISER-1-5 & 100.00 \\
\hline HDAT & (null) & U102-RISER-1-6 & 124.00 \\
\hline HDAT & (null) & U102-RISER-1-7 & 148.00 \\
\hline HDAT & (null) & U102-RISER-1-8 & 172.00 \\
\hline HDAT & (null) & U102-RISER-1-9 & 196.00 \\
\hline HDAT & (nuil) & U103-RISER-1-1 & 21.36 \\
\hline HDAT & (null) & U103-RISER-1-10 & 261.36 \\
\hline HDAT & (null) & U103-RISER-1-11 & 309.36 \\
\hline HDAT & (null) & U103-RISER-1-2 & 45.36 \\
\hline HDAT & (null) & U103-RISER-1-3 & 69.36 \\
\hline HDAT & (nuil) & U103-RISER-1-4 & 93.36 \\
\hline HDAT & (nuli) & U103-RISER-1-5 & 117.36 \\
\hline HDAT & (nuil) & U103-RISER-1-6 & 141.36 \\
\hline HDAT & (null) & U103-RISER-1-7 & 165.36 \\
\hline HDAT & (nuil) & U103-RISER-1-8 & 189.36 \\
\hline HDAT & (null) & U103-RISER-1-9 & 213.36 \\
\hline HDAT & (null) & U105-RISER-1-1 & 21.36 \\
\hline HDAT & (null) & U105-RISER-1-10 & 261.36 \\
\hline HDAT & (null) & U105-RISER-1-11 & 309.36 \\
\hline HDAT & (null) & U105-RISER-1-2 & 45.36 \\
\hline HDAT & (null) & U105-RISER-1-3 & 69.36 \\
\hline HDAT & (null) & U105-RISER-1-4 & 93.36 \\
\hline HDAT & (null) & U105-RISER-1-5 & 117.36 \\
\hline HDAT & (nuil) & U105-RISER-1-6 & 141.36 \\
\hline HDAT & (null) & U105-RISER-1-7 & 165.36 \\
\hline HDÁT & (nuil) & U105-RISER-1-8 & 189.36 \\
\hline HDAT & (nuil) & U105-RISER-1-9 & 213.36 \\
\hline HDAT & (nuli) & U106-RISER-1-1 & 20.16 \\
\hline HDAT & (null) & U106-RISER-1-10 & 260.16 \\
\hline HDAT & (null) & U106-RISER-1-11 & 308.16 \\
\hline HDAT & (nult) & U106-RISER-1-2 & 44.16 \\
\hline HDAT & (nuil) & U106-RISER-1-3 & 68.16 \\
\hline HOAT & (null) & U106-RISER-1.4 & 92.16 \\
\hline HDAT & (nuil) & U106-RISER-1-5 & 116.16 \\
\hline HDAT & (null) & U106-RISER-1-6 & 140.16 \\
\hline HDAT & (null) & U106-RISER-1-7 & 164.16 \\
\hline HDAT & (null) & U106-RISER-1-8 & 188.16 \\
\hline HDAT & (null) & U106-RISER-1-9 & 212.16 \\
\hline HDAT & (null) & U107-RISER-1-1 & 4.00 \\
\hline HDAT & (null) & U107-RISER-1-10 & 220.00 \\
\hline HDAT & (null) & U107-RISER-1-11 & 244.00 \\
\hline HDAT & (null) & U107-RISER-1-12 & 268.00 \\
\hline HDAT & (null) & U107-RISER-1-13 & 316.0 \\
\hline
\end{tabular}




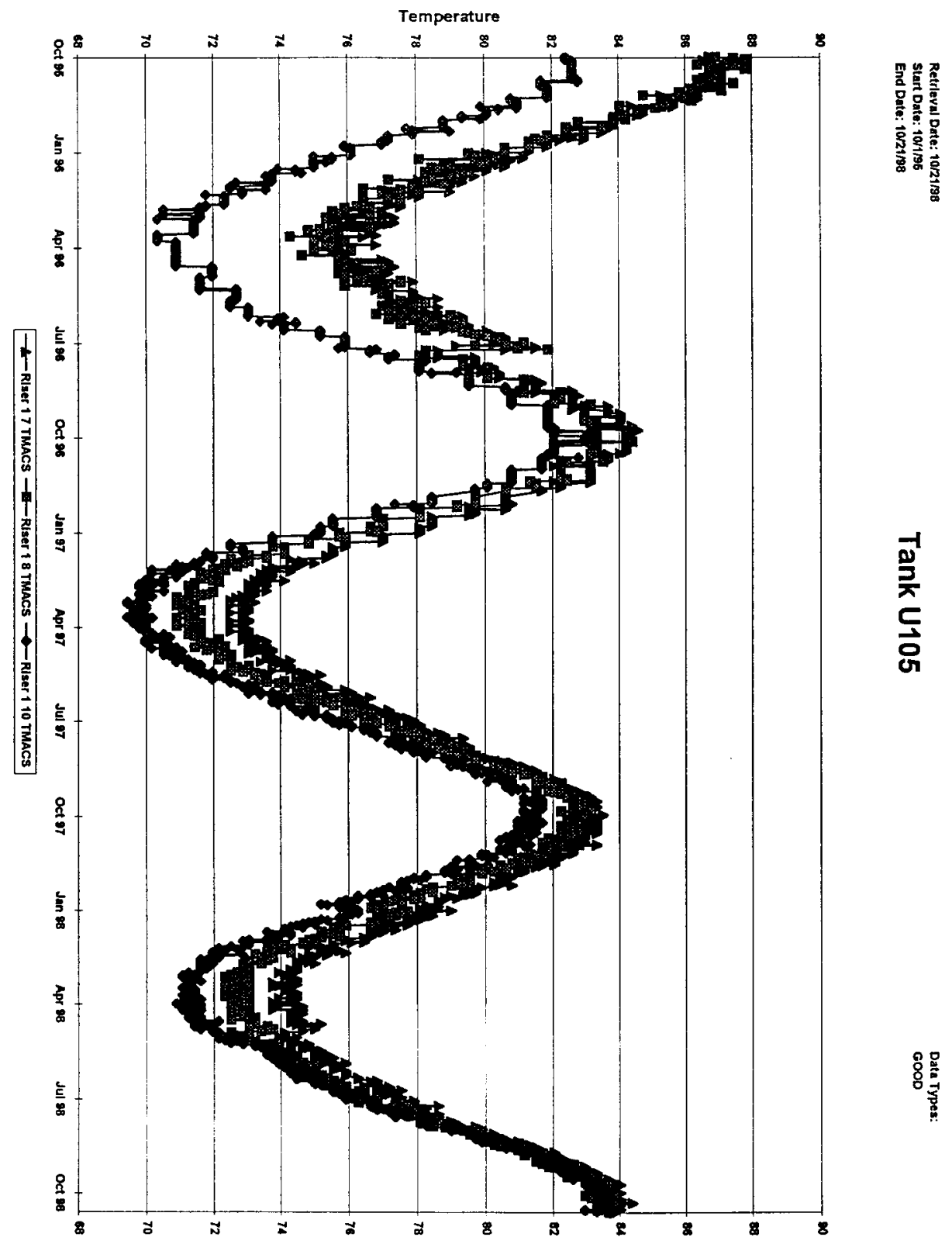


Surface Level (Inches)

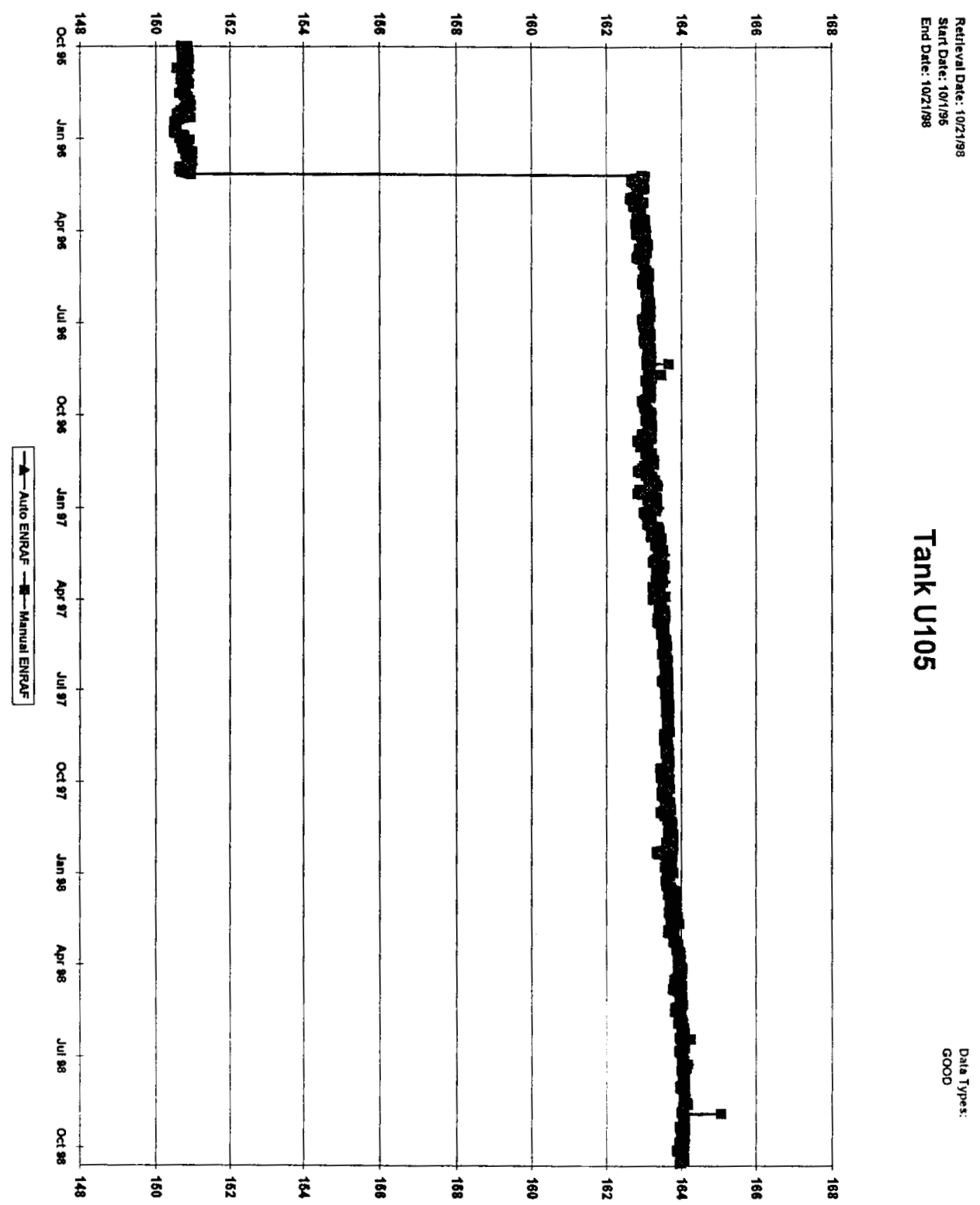




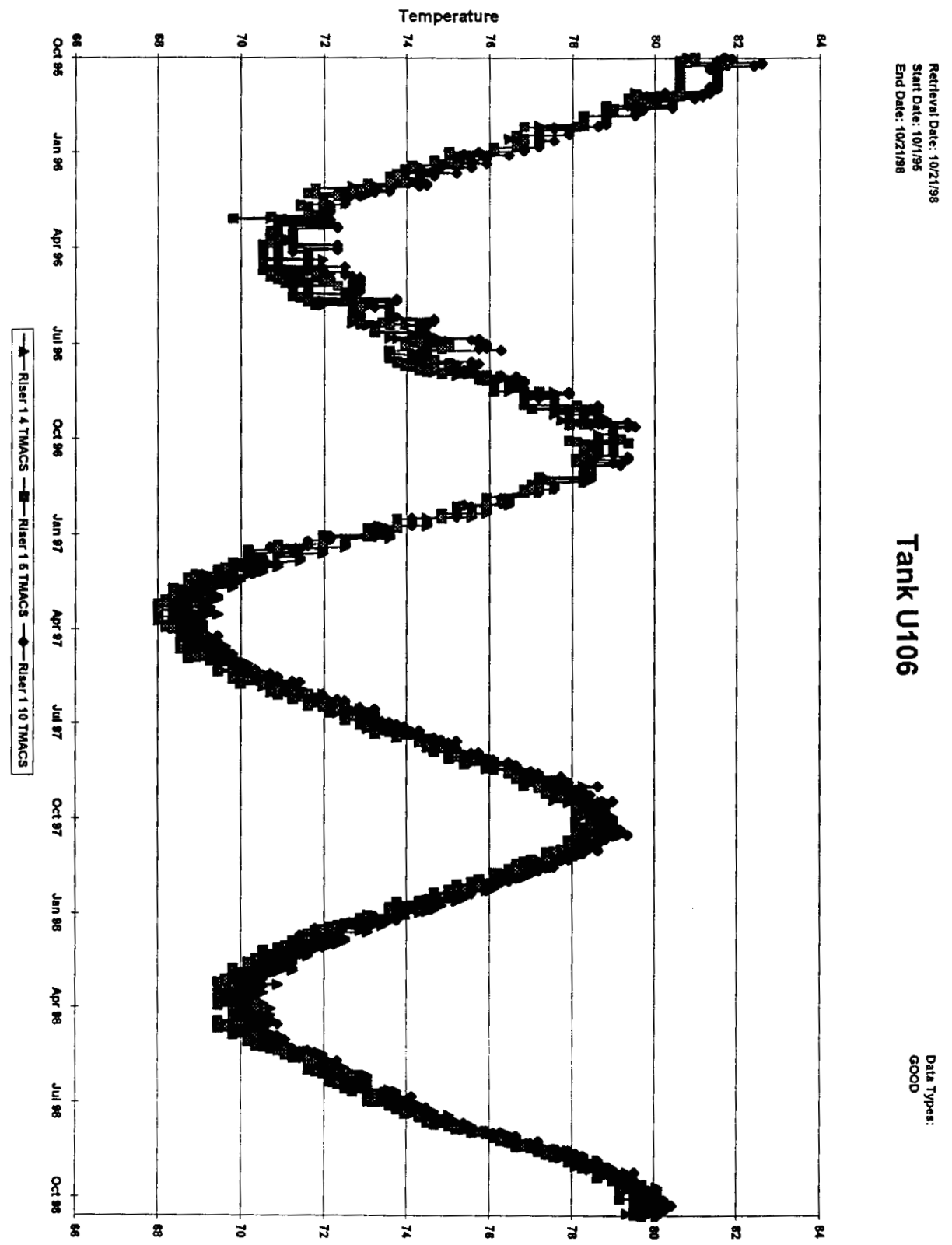




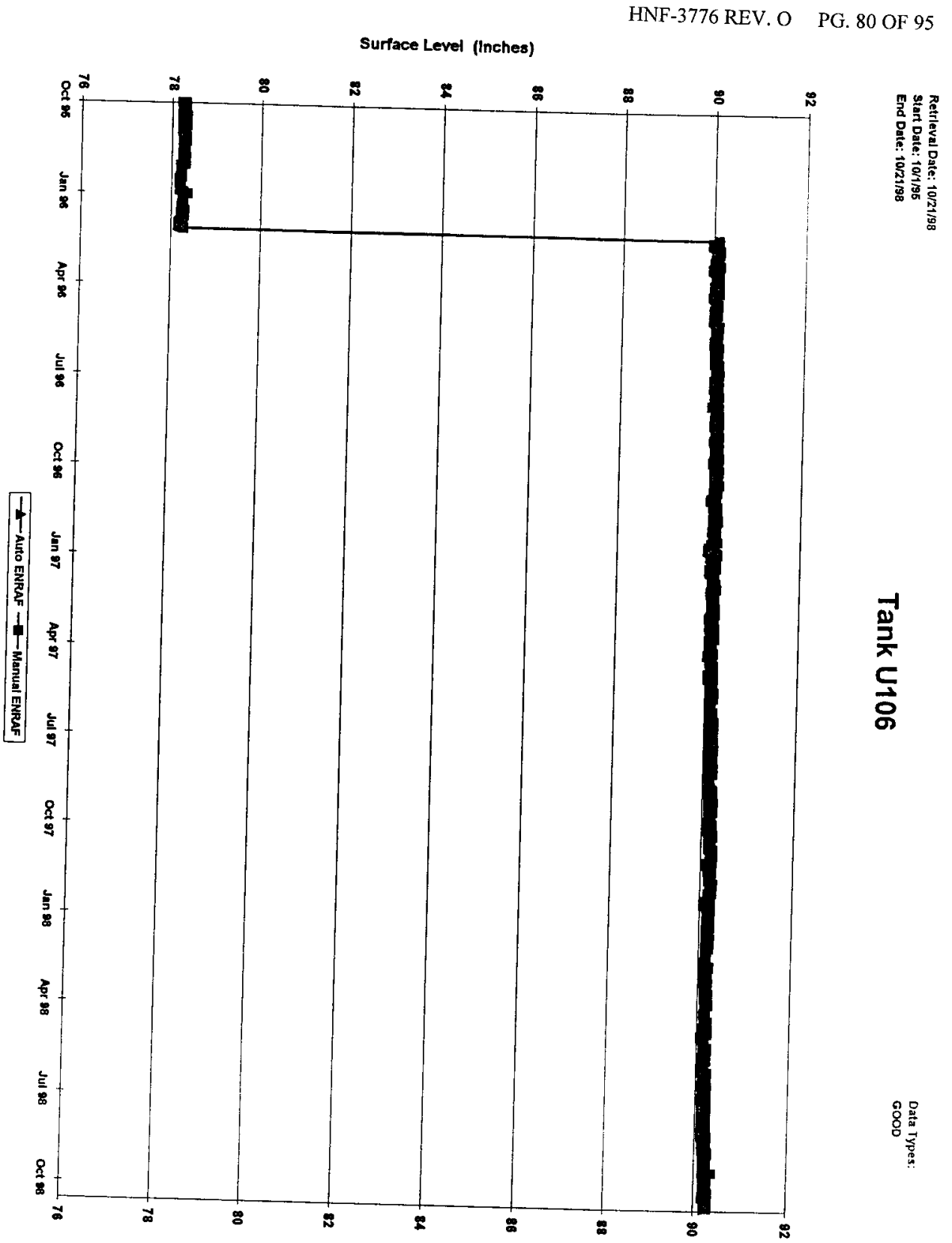




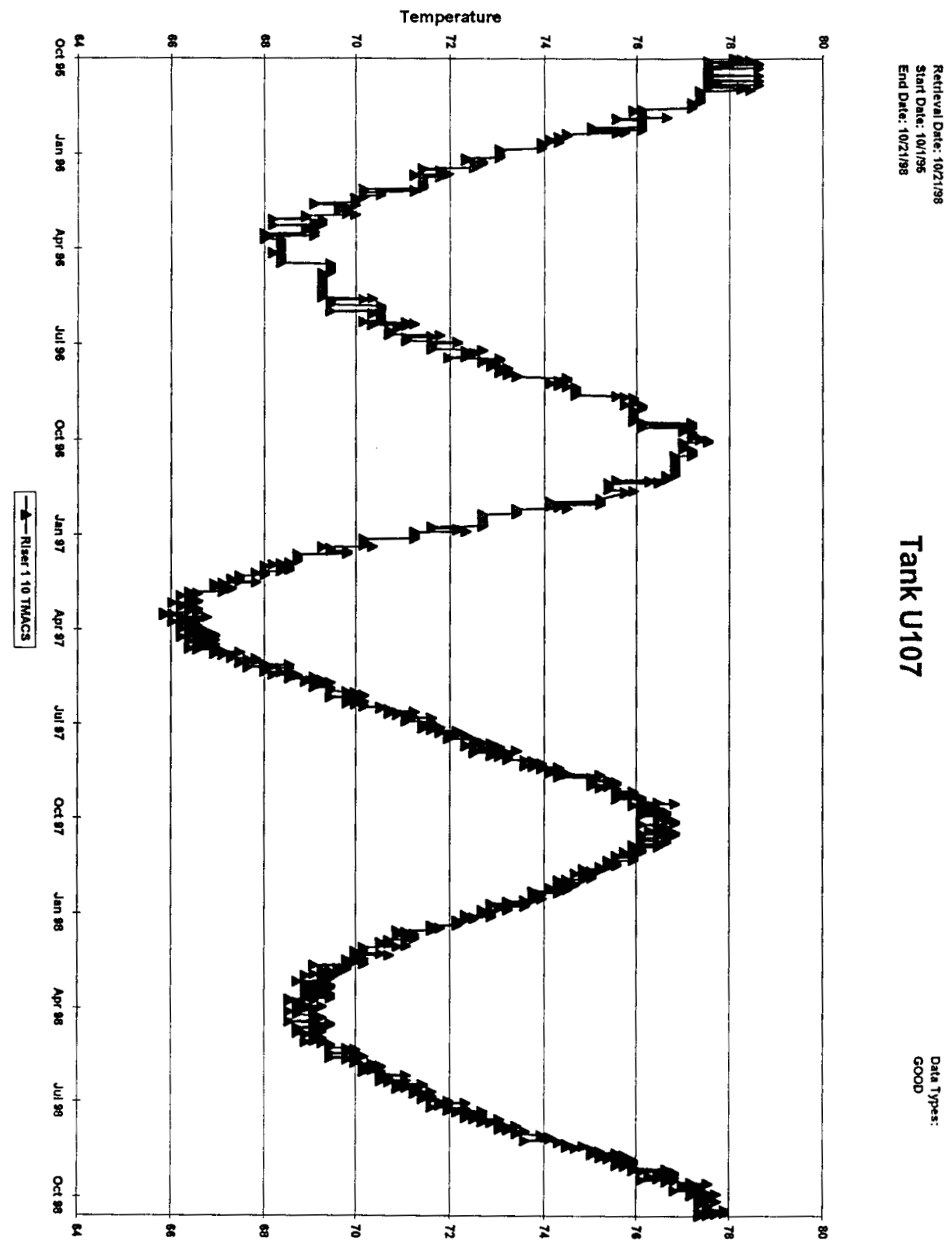


INF-3776 REV. O

PG. 82 OF 95

Surface Level (Inches)

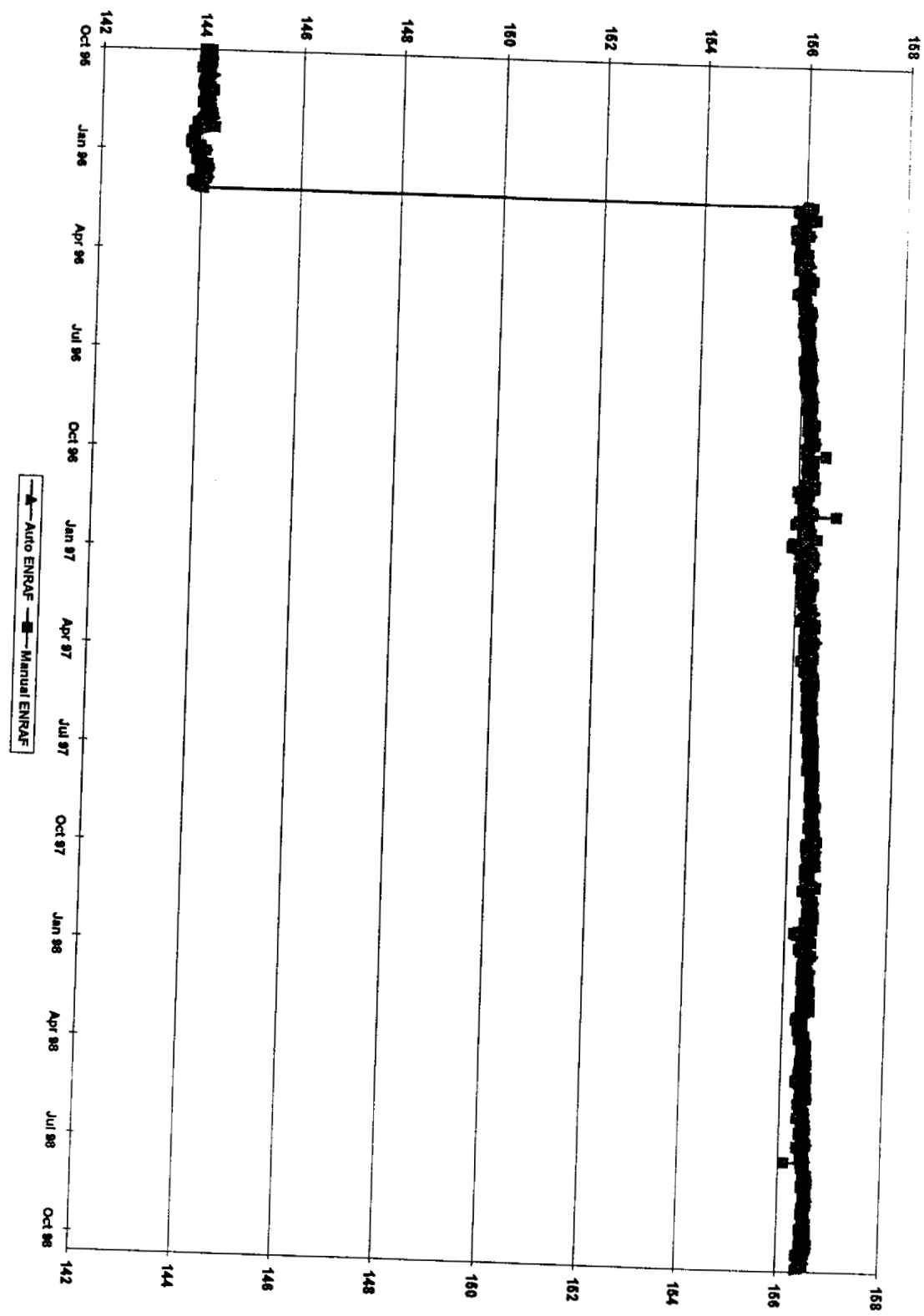

$\frac{}{2}$

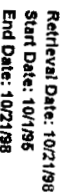

串题 
Sheet1

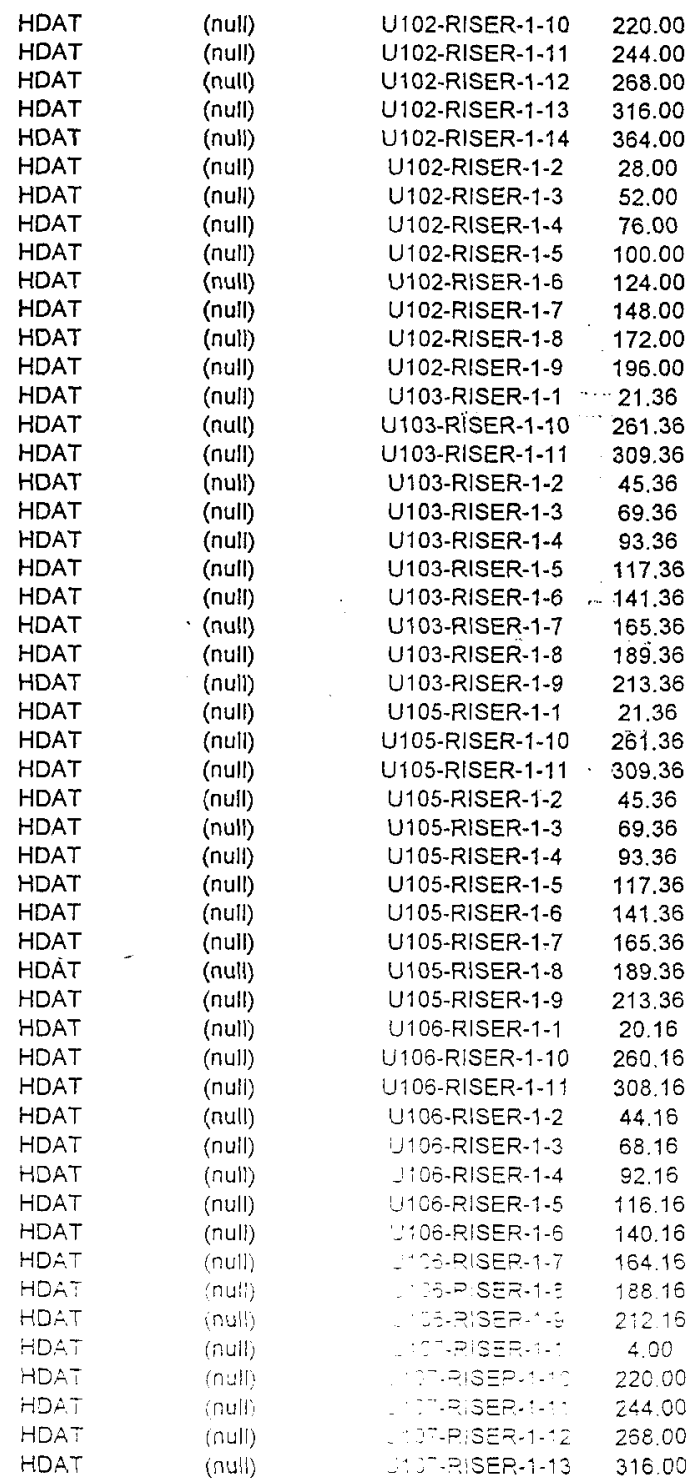




\begin{tabular}{|c|c|c|c|}
\hline HDAT & (nuIi) & U107-RISER-1-14 & 364.00 \\
\hline HDAT & (nuli) & U107-RISER-1-2 & 28.00 \\
\hline HDAT & (null) & U107-RISER-1-3 & 52.00 \\
\hline HDAT & (null) & U107-RISER-1-4 & 76.00 \\
\hline HDAT & (nuli) & U107-RISER-1-5 & 100,00 \\
\hline HDAT & (null) & U107-RISER-1-6 & 124.00 \\
\hline HDAT & (null) & U107-RISER-1-7 & 148.00 \\
\hline HDAT & (null) & U107-RISER-1-8 & 172.00 \\
\hline HDAT & (nuli) & U107-RISER-1-9 & 196.00 \\
\hline HDAT & (null) & U108-RISER-1-1 & 4.00 \\
\hline HDAT & (null) & U108-RISER-1-10 & 220.00 \\
\hline HDAT & (null) & Ut08-RISER-1-11 & 244.00 \\
\hline HDAT & (nuil) & U108-RISER-1-12 & 268.00 \\
\hline HDAT & (null) & U108-RISER-1-13 & 316.00 \\
\hline HDAT & (null) & U108-RISER-1-14 & 364.00 \\
\hline HDAT & (null) & U108-RISER-1-2 & 28.00 \\
\hline HDAT & (null) & U108-RISER-1-3 & 52.00 \\
\hline HDAT & (null) & U108-RISER-1-4 & 76.00 \\
\hline HDAT & (null) & U108-RISER-1-5 & 100.00 \\
\hline HDAT & (nuil) & U108-RISER-1-6 & 124.00 \\
\hline HDAT & (null) & U108-RISER-1-7 & 148.00 \\
\hline HDAT & (null) & U108-RISER-1-8 & 172.00 \\
\hline HDAT & (nuil) & U108-RISER-1-9 & 196.00 \\
\hline HDAT & (nuII) & U109-RISER-1-1 & 4.00 \\
\hline HDAT & (null) & U109-RISER-1-10 & 220.00 \\
\hline HDAT & (null) & U109-RISER-1-11 & 244.00 \\
\hline HDAT & (nuil) & U109-RISER-1-12 & 268.00 \\
\hline HDAT & (null) & U109-RISER-1-13 & 316.00 \\
\hline HDAT & (null) & U109-RISER-1-14 & 364.00 \\
\hline HDAT & (null) & U109-RISER-1-2 & 28.00 \\
\hline HDAT & (null) & U109-RISER-1-3 & 52.00 \\
\hline HDAT & (null) & U109-RISER-1-4 & 76.00 \\
\hline HDAT & (null) & U109-RISER-1-5 & 100.00 \\
\hline HDAT & (null) & U109-RISER-1-6 & 124.00 \\
\hline HDAT & (null) & U109-RISER-1-7 & 148.00 \\
\hline HDAT & (nuill) & U109-RISER-1-8 & 172.00 \\
\hline HDAT & (null) & U109-RISER-1-9 & 196.00 \\
\hline HDAT & (null) & U110-RISER-1-1 & 4.00 \\
\hline HDAT & (null) & U110-RISER-1-10 & 220.00 \\
\hline HDAT & (null) & I110-RISER-1-11 & 244.00 \\
\hline HDAT & (null) & U110-RISER-1-12 & 268.00 \\
\hline HDAT & (null) & L1110-RISER-1-13 & 316.00 \\
\hline HDAT & (nuli) & U:10-RISER-1-14 & 364.00 \\
\hline HDAT & (nuli) & $-10-R I S E R+1-2$ & 28.00 \\
\hline HDAT & (null) & - 10-RISER-1-3 & 52.00 \\
\hline HDAT & (nuli) & - OU-RISER- $-A$ & 76.00 \\
\hline HDAT & (null) & $-\cdots$-RISER- + -E & 100.00 \\
\hline HDAT & (null) & $\therefore$ - & 124.00 \\
\hline HDAT & (nult) & $-1 \hat{U}-F$ ISER- $\hat{1}-\bar{i}$ & 148.00 \\
\hline HDAT & (null) & -110-RISER-1-3 & 172.00 \\
\hline HDAT & (nuili) & -110-RISER-1-9 & 196.00 \\
\hline
\end{tabular}


HNF-3776 REV. O PG. 85 OF 95

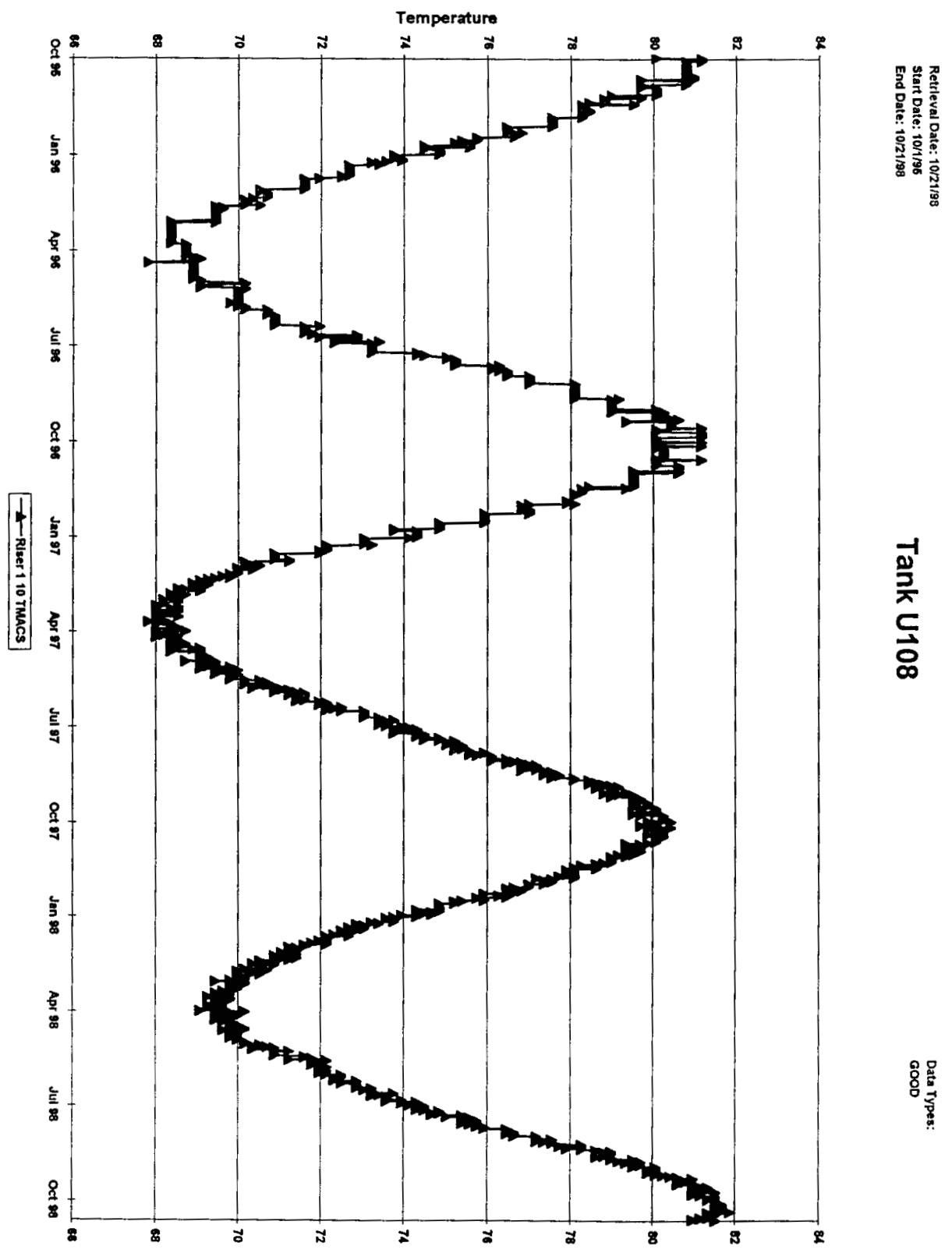




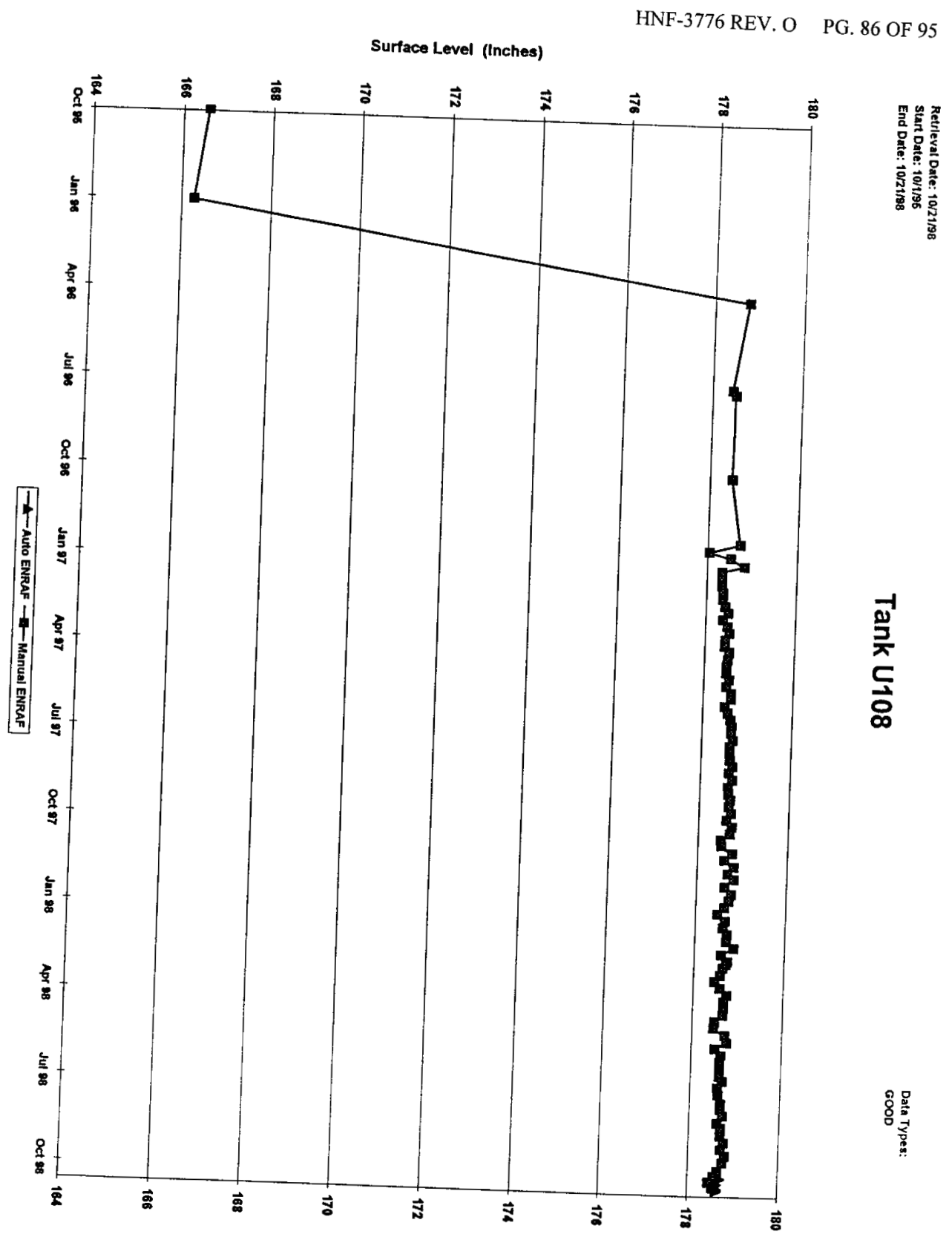


Sheett

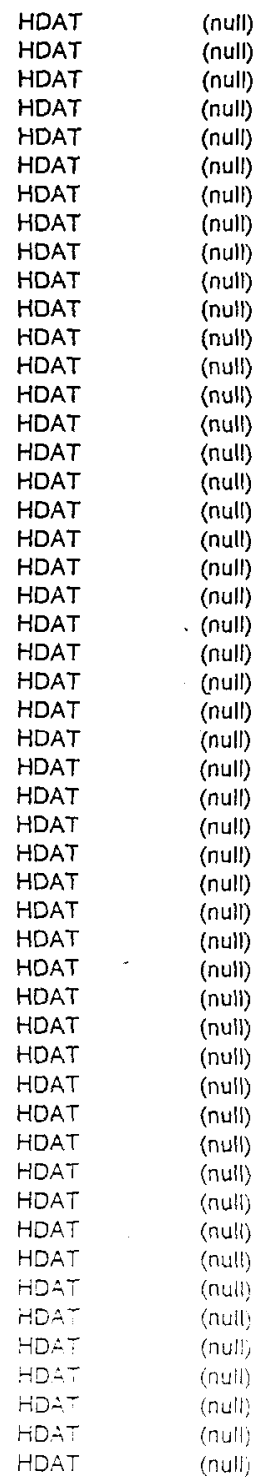

\begin{tabular}{|c|c|}
\hline 列, & \\
\hline U107-RISER-1-2 & \\
\hline U107-RISER-1-3 & 2.00 \\
\hline U107-RISER-1-4 & 76.00 \\
\hline U107-RISER-1-5 & 100.00 \\
\hline U107-RISER-1-6 & 124.00 \\
\hline U107-RISER-1-7 & 148.00 \\
\hline U107-RISER-1-8 & 172.00 \\
\hline U107-RISER-1-9 & 196.00 \\
\hline U108-RISER-1-1 & 4.00 \\
\hline 108-RISER-1-10 & 220.00 \\
\hline 108-RISER-1-11 & 244.00 \\
\hline 108-RISER-1-12 & 268.00 \\
\hline 108-RISER-1-13 & .316 .00 \\
\hline 108-RISER-1-14 & 364.00 \\
\hline ISER-1-2 & 28.00 \\
\hline ISER-1-3 & 52.00 \\
\hline ISER-1-4 & 76.00 \\
\hline SER-1.5 & 100.00 \\
\hline SER-1-6 & 124.00 \\
\hline ISER-1-7 & 148.00 \\
\hline ISER-1-8 & 172.00 \\
\hline SER-1-9 & 196.00 \\
\hline$S E R-1-1$ & 4.00 \\
\hline$E R-1-10$ & 220.00 \\
\hline SER-1-11 & 244.00 \\
\hline SER-1-12 & 268.00 \\
\hline SER-1-13 & 316.00 \\
\hline SER-1-14 & 364.00 \\
\hline SER-1-2 & 28.00 \\
\hline SER-1-3 & 52.00 \\
\hline SER-1-4 & 6.00 \\
\hline ERR-1-5 & 100.00 \\
\hline ER-1-6 & 124.00 \\
\hline ERR-1-7 & 148.00 \\
\hline SER-1-8 & 172.00 \\
\hline ER-1-9 & 196.00 \\
\hline SER-1-1 & 4.00 \\
\hline SER-1-10 & 220.00 \\
\hline U110-RISER-1-11 & 244.00 \\
\hline U: 10-RISER-1-12 & 268.00 \\
\hline U:10-RISER-1-13 & 316.00 \\
\hline U110-RISER-1-14 & 364.00 \\
\hline : 10 -RISER-1.2 & 28.00 \\
\hline -11S-R!SEF-A & 52.00 \\
\hline$\because$ ZSEF & 76.00 \\
\hline$-\cdots R-P B S P^{*}=$ & 100.00 \\
\hline APIBEF:- & 124.00 \\
\hline$\because P S E F-1-$ & 148.00 \\
\hline$-\cdots-3$ SER-1-z & 172.00 \\
\hline$-1 C-P I S E R-1-\hat{a}$ & 190.00 \\
\hline
\end{tabular}


HNF-3776 REV. O PG. 88 OF 95

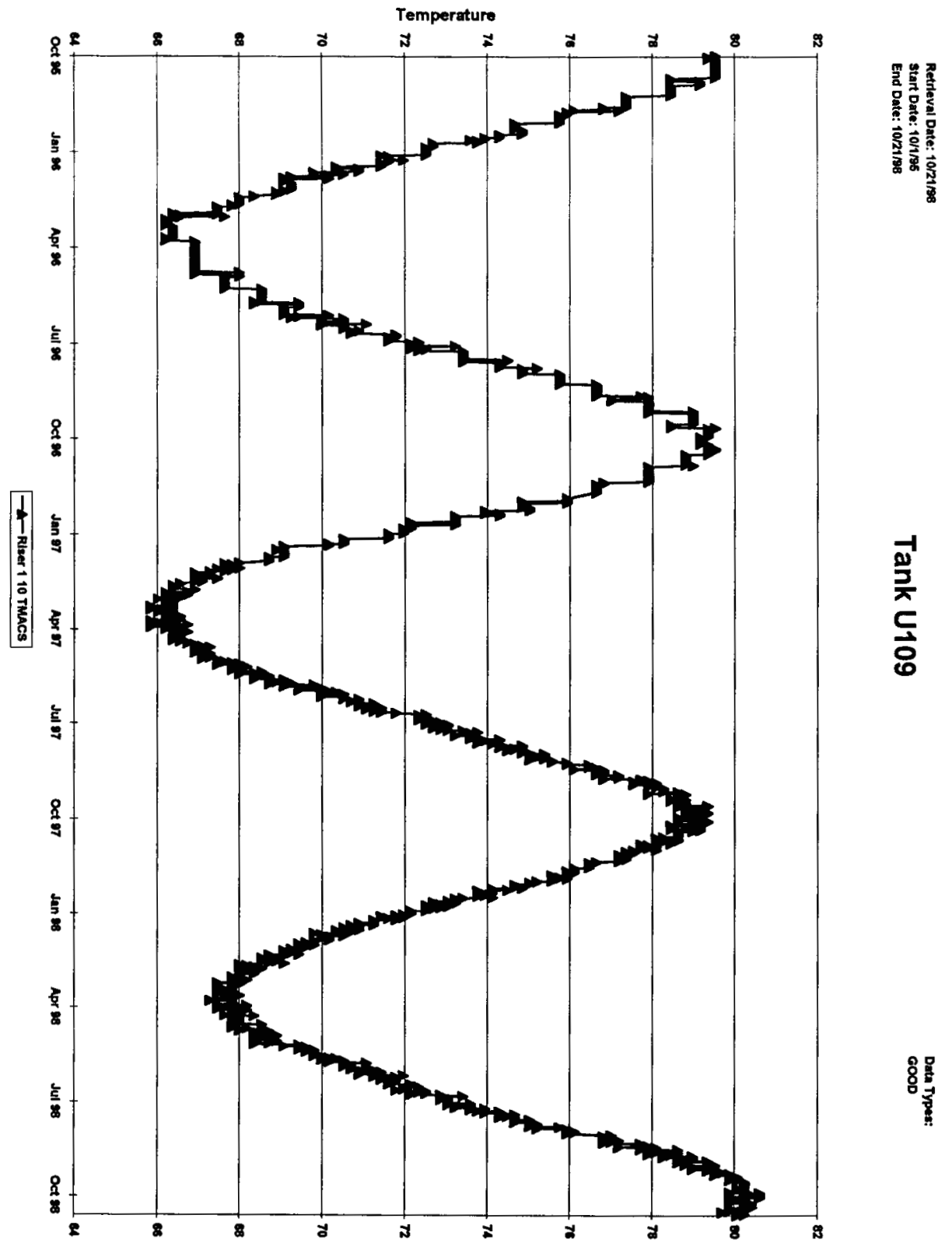




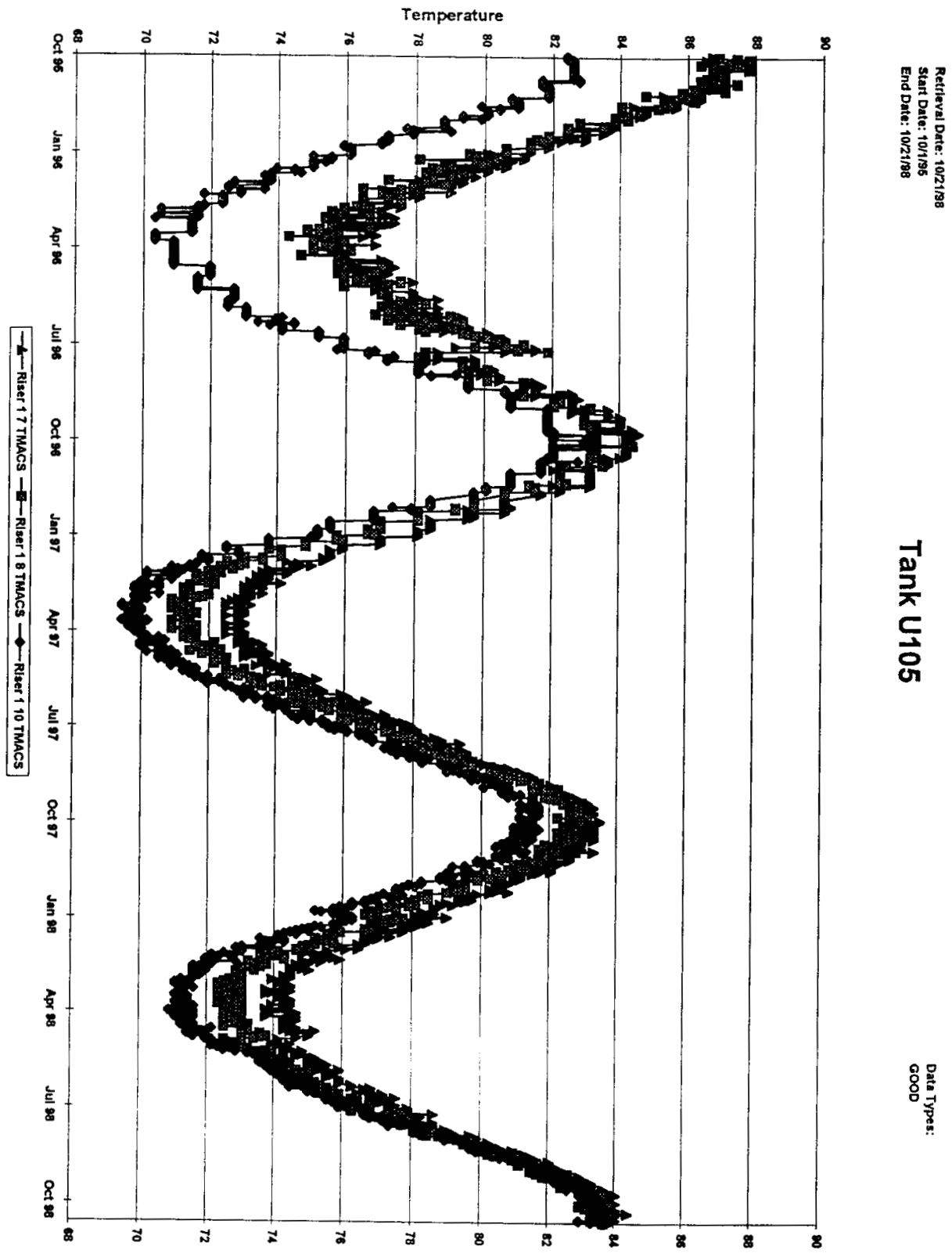


HNF-3776 REV. O PG. 90 OF 95

Surface Level (Inches)

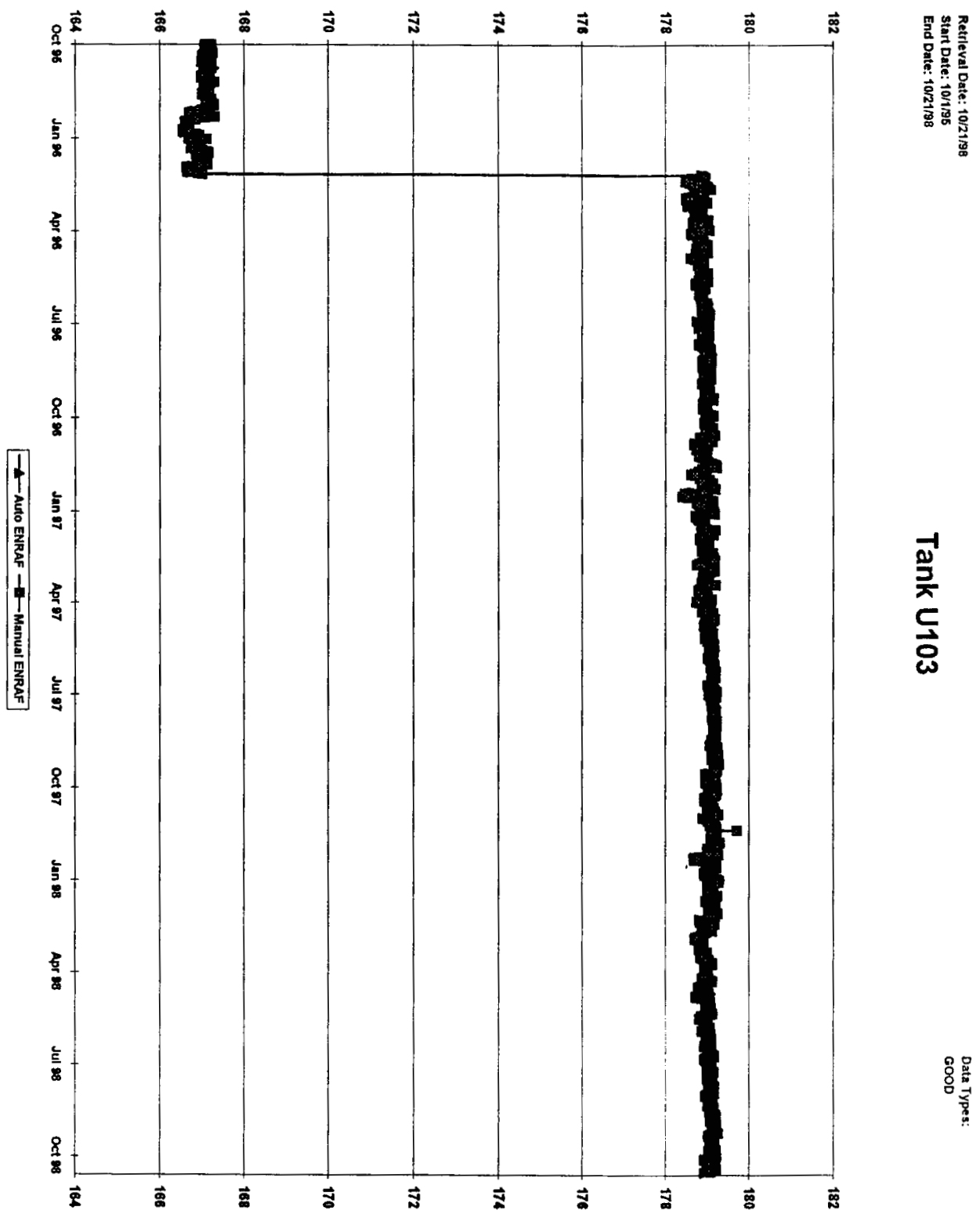


HNF-3776 REV. O PG. 91 OF 95

Surface Level (Inches)

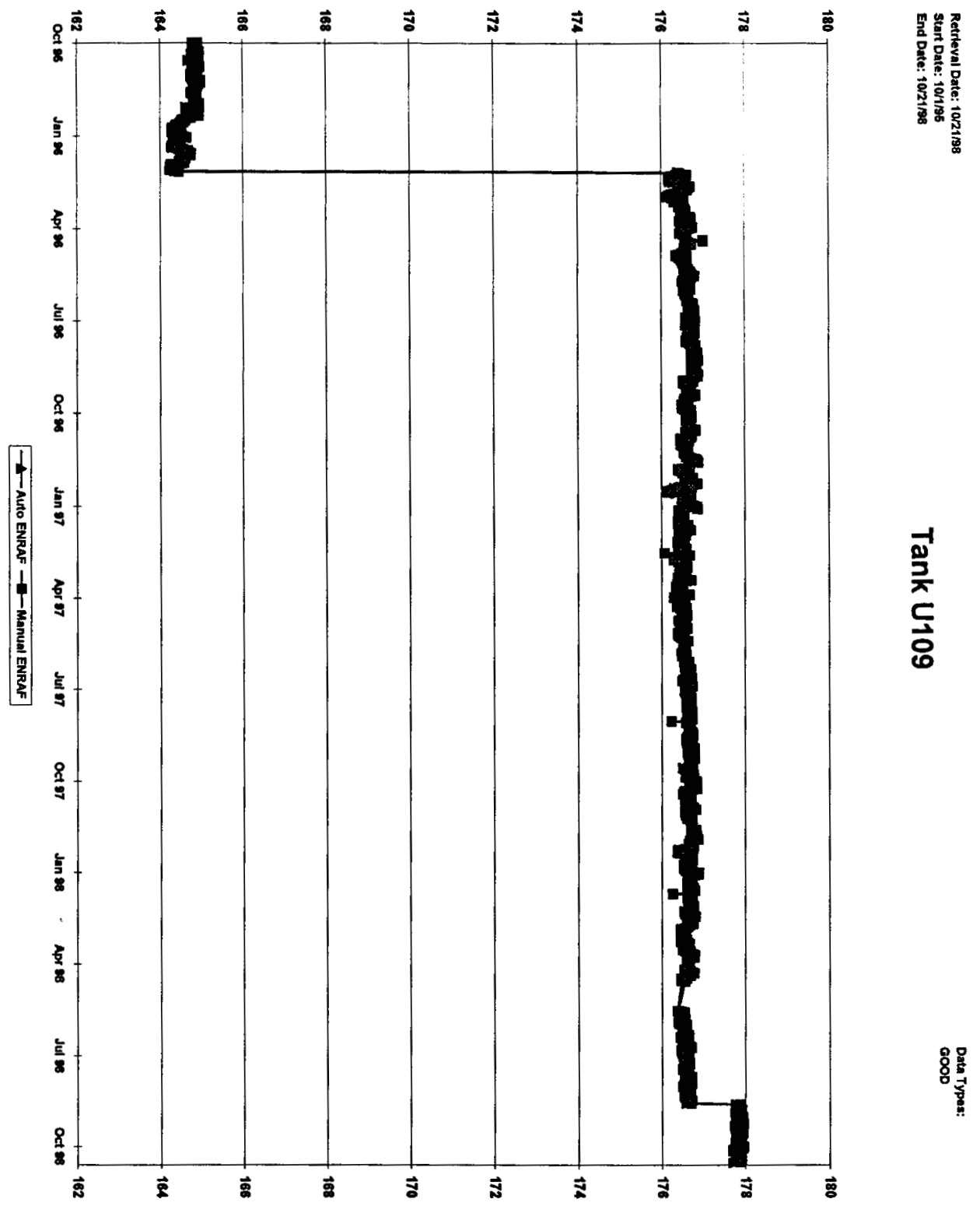




\begin{tabular}{|c|c|c|c|c|}
\hline HDAT & & (nuil) & U107-RISER-1-14 & 364.00 \\
\hline HDAT & & (null) & U107-RISER-1-2 & 28.00 \\
\hline HDAT & & (nuil) & U107-RISER-1-3 & 52.00 \\
\hline HDAT & & (null) & U107-RISER-1-4 & 76.00 \\
\hline HDAT & & (null) & U107-RISER-1-5 & 100.00 \\
\hline HDAT & & (null) & U107-RISER-1-6 & 124,00 \\
\hline HDAT & & (null) & U107-RISER-1-7 & 148.00 \\
\hline HDAT & & (null) & U107-RISER-1-8 & 172.00 \\
\hline HDAT & & (null) & U107-RISER-1-9 & 196.00 \\
\hline HDAT & & (null) & U108-RISER-1-1 & 4.00 \\
\hline HDAT & & (null) & U108-RISER-1-10 & 220.00 \\
\hline HDAT & & (null) & U108-RISER-1-11 & 244.00 \\
\hline HDAT & & (nuli) & U108-RISER-1-12 & 268.00 \\
\hline HDAT & & (nuill) & U108-RISER-1-13 & .316 .00 \\
\hline HDAT & & (null) & U108-RISER-1-14 & 364.00 \\
\hline HDAT & & (nuil) & U108-RISER-1-2 & 28.00 \\
\hline HDAT & & (null) & U108-RISER-1-3 & 52.00 \\
\hline HDAT & & (null) & U108-RISER-1-4 & 76.00 \\
\hline HDAT & & (null) & U108-RISER-1-5 & 100.00 \\
\hline HDAT & & (nuil) & U108-RISER-1-6 & 124.00 \\
\hline HDAT & & (null) & U108-RISER-1-7 & 148.00 \\
\hline HDAT & & (null) & U108-RISER-1-8 & 172.00 \\
\hline HDAT & & (nuil) & U108-RISER-1-9 & 196.00 \\
\hline HDAT & & (null) & U109-RISER-1-1 & 4.00 \\
\hline HDAT & & (null) & U109-RISER-1-10 & 220.00 \\
\hline HDAT & & (null) & U109-RISER-1-11 & 244.00 \\
\hline HDAT & & (null) & U109-RISER-1-12 & 268.00 \\
\hline HDAT & & (nuli) & U109-RISER-1-13 & 316.00 \\
\hline HDAT & & (null) & U109-RISER-1-14 & 364.00 \\
\hline HDAT & & (nuli) & U109-RISER-1-2 & 28.00 \\
\hline HDAT & & (null) & U109-RISER-1-3 & 52.00 \\
\hline HDAT & & (nuil) & U109-RISER-1-4 & 76.00 \\
\hline HDAT & & (null) & U109-RISER-1-5 & 100.00 \\
\hline HDAT & - & (null) & U109-RISER-1-6 & 124.00 \\
\hline HDAT & & (null) & U109-RISER-1-7 & 148.00 \\
\hline HDAT & & (nuIl) & U109-RISER-1-8 & 172.00 \\
\hline HDAT & & (nuil) & U109-RISER-1-9 & 196.00 \\
\hline HDAT & & (null) & U110-RISER-1-1 & 4.00 \\
\hline HDAT & & (null) & U110-RISER-1-10 & 220.00 \\
\hline HDAT & & (null) & U110-RISER-1-11 & 244.00 \\
\hline HDAT & & (null) & U110-RISER-1-12 & 268.00 \\
\hline HDAT & & (null) & U $110-R I S E R-1-13$ & 316.00 \\
\hline HDAT & & (null) & U110-RISER- $1-14$ & 364.00 \\
\hline HDAT & & (null) & U110-RISER-1-2 & 28.00 \\
\hline HDAT & & (nuli) & U110-RISER-1-3 & 52.00 \\
\hline HDAT & & (null) & U110-RISER-1-4 & 76.00 \\
\hline HDAT & & (null) & U110-RISER-1-5 & 100.00 \\
\hline HDAT & & (nuil) & U110-RISER-1-6 & 124,00 \\
\hline HDAT & & (nuli) & U110-RISER-1-7 & 148.00 \\
\hline HDAT & & (null) & U110-RISER-1-8 & 172.00 \\
\hline HDAT & & (null) & U110-RISER-1-9 & 196.00 \\
\hline
\end{tabular}


HNF-3776 REV. O PG. 93 OF 95

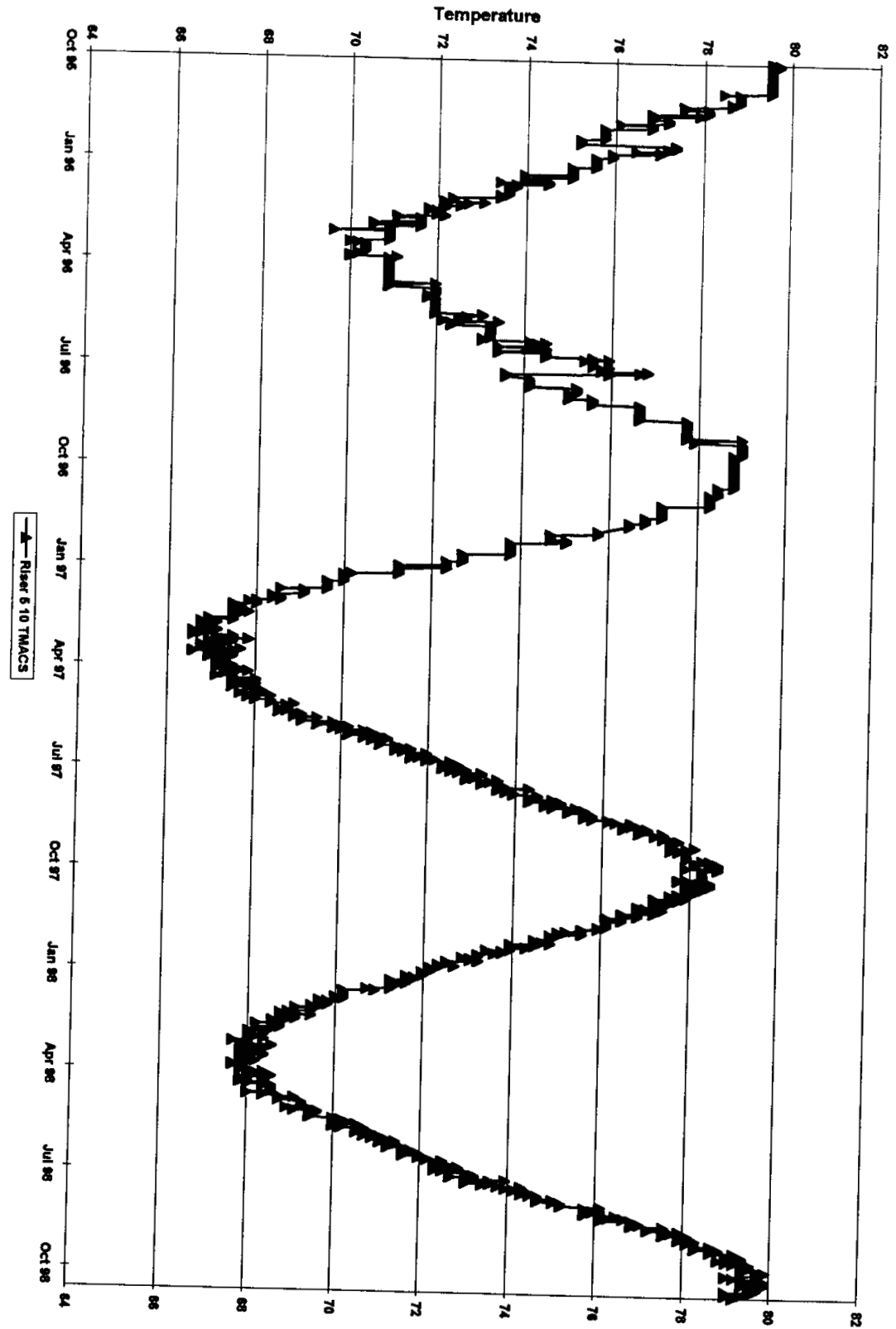

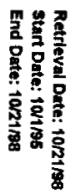

高

8 


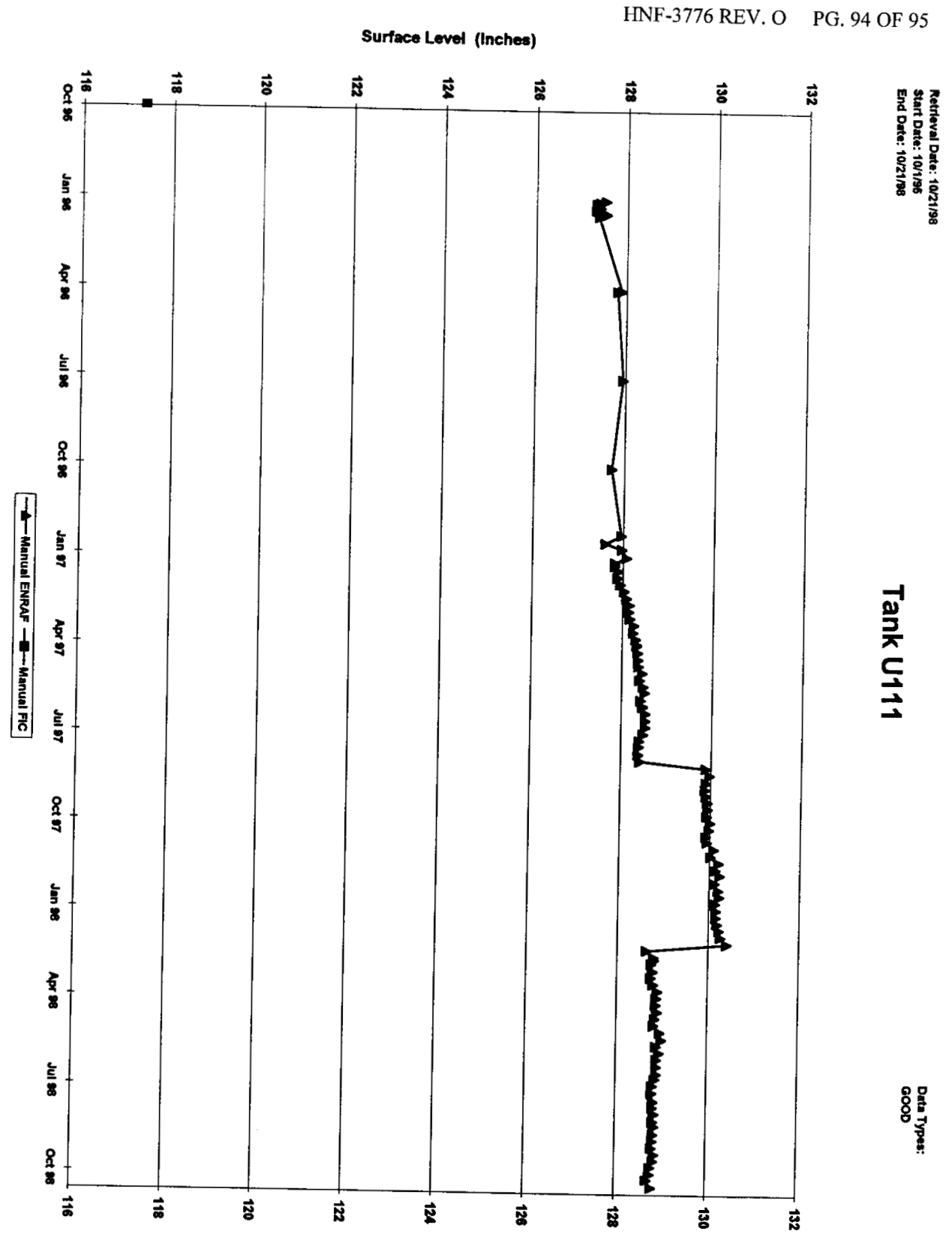


Sheet1

\begin{tabular}{|c|c|c|c|}
\hline HDAT & (null) & U111-RISER-5-1 & 4.00 \\
\hline HDAT & (null) & U111-RISER-5-10 & 220.00 \\
\hline HDAT & (null) & U111-RISER-5-11 & 244.00 \\
\hline HDAT & (null) & U111-RISER-5-12 & 268.00 \\
\hline HDAT & (null) & U111-RISER-5-13 & 316.00 \\
\hline HDAT & (null) & U111-RISER-5-14 & 364.00 \\
\hline HDAT & (null) & U111-RISER-5-2 & 28.00 \\
\hline HDAT & (nulli) & U111-RISER-5-3 & 52.00 \\
\hline HDAT & (null) & U111-RISER-5-4 & 76.00 \\
\hline HDAT & (null) & U111-RISER-5-5 & 100.00 \\
\hline HDAT & (nuil) & U111-RISER-5-6 & 124.00 \\
\hline HDAT & (null) & U111-RISER-5-7 & 148.00 \\
\hline HDAT & (null) & U111-RISER-5-8 & 172.00 \\
\hline HDAT & (null) & U111-RISER-5-9 & 196.00 \\
\hline HDAT & (null) & U112-RISER-5-1 & 4.00 \\
\hline HDAT & (null) & U112-RISER-5-10 & 220.00 \\
\hline HDAT & (null) & U112-RISER-5-11 & 244.00 \\
\hline HDAT & (null) & U112-RISER-5-12 & 268.00 \\
\hline HDAT & (null) & U112-RISER-5-13 & 316.00 \\
\hline HDAT & (null) & U112-RISER-5-14 & 364.00 \\
\hline HDAT & (null) & U112-RISER-5-2 & 28.00 \\
\hline HDAT & (nuil) & U112-RISER-5-3 & 52.00 \\
\hline HDAT & (null) & U112-RISER-5-4 & 76.00 \\
\hline HDAT & (nuli) & U112-RISER-5-5 & 100.00 \\
\hline HDAT & (null) & U112-RISER-5-6 & 124.00 \\
\hline HDAT & (null) & U112-RISER-5-7 & 148.00 \\
\hline HDAT & (nuil) & U112-RISER-5-8 & 172.00 \\
\hline HDAT & (null) & U112-RISER-5-9 & 196.00 \\
\hline HDAT & (null) & U201-RISER-4-1 & 4.00 \\
\hline HDAT & (null) & U201-RISER-4-10 & 220.00 \\
\hline HDAT & (null) & U201-RISER-4-11 & 244.00 \\
\hline HDAT & (null) & U201-RISER-4-12 & 268.00 \\
\hline HDAT & (null) & U201-RISER-4-13 & 316.00 \\
\hline HDAT & (null) & U201-RISER-4-14 & 364.00 \\
\hline HDAT & (nuli) & U201-RISER-4-2 & 28.00 \\
\hline HDAT & (null) & U201-RISER-4-3 & 52.00 \\
\hline HDAT & (nuil) & U201-RISER-4-4 & 76.00 \\
\hline HDAT & (null) & U201-RISER-4-5 & 100.00 \\
\hline HDAT & (nuli) & U201-RISER-4-6 & 124.00 \\
\hline HDAT & (null) & U201-RISER-4-7 & 148.00 \\
\hline HDAT & (null) & U201-RISER-4-8 & 172.00 \\
\hline HDAT & (null) & U201-RISER-4-9 & 196.00 \\
\hline HDAT & (null) & U202-RISER-4-1 & 4.00 \\
\hline HDAT & (nuil) & U202-RISER-4-10 & 220.00 \\
\hline HDAT & (null) & U202-RISER-4-11 & 244.00 \\
\hline HDAT & (null) & U202-RISER-4-12 & 268.00 \\
\hline HDAT & (null) & U202-RISER-4-13 & 316.00 \\
\hline HDAT & (null) & U202-RISER-4-14 & 364.00 \\
\hline HDAT & (null) & U202-RISER-4-2 & 28.00 \\
\hline HDAT & (null) & U202-RISER-4-3 & 52.00 \\
\hline HDAT & (null) & U202-RISER-4-4 & 76.00 \\
\hline
\end{tabular}




\section{DISTRIBUTION SHEET}

To

Distribution

\section{Project Title Work Order}

Stabilization/Portable Exhauster Mods

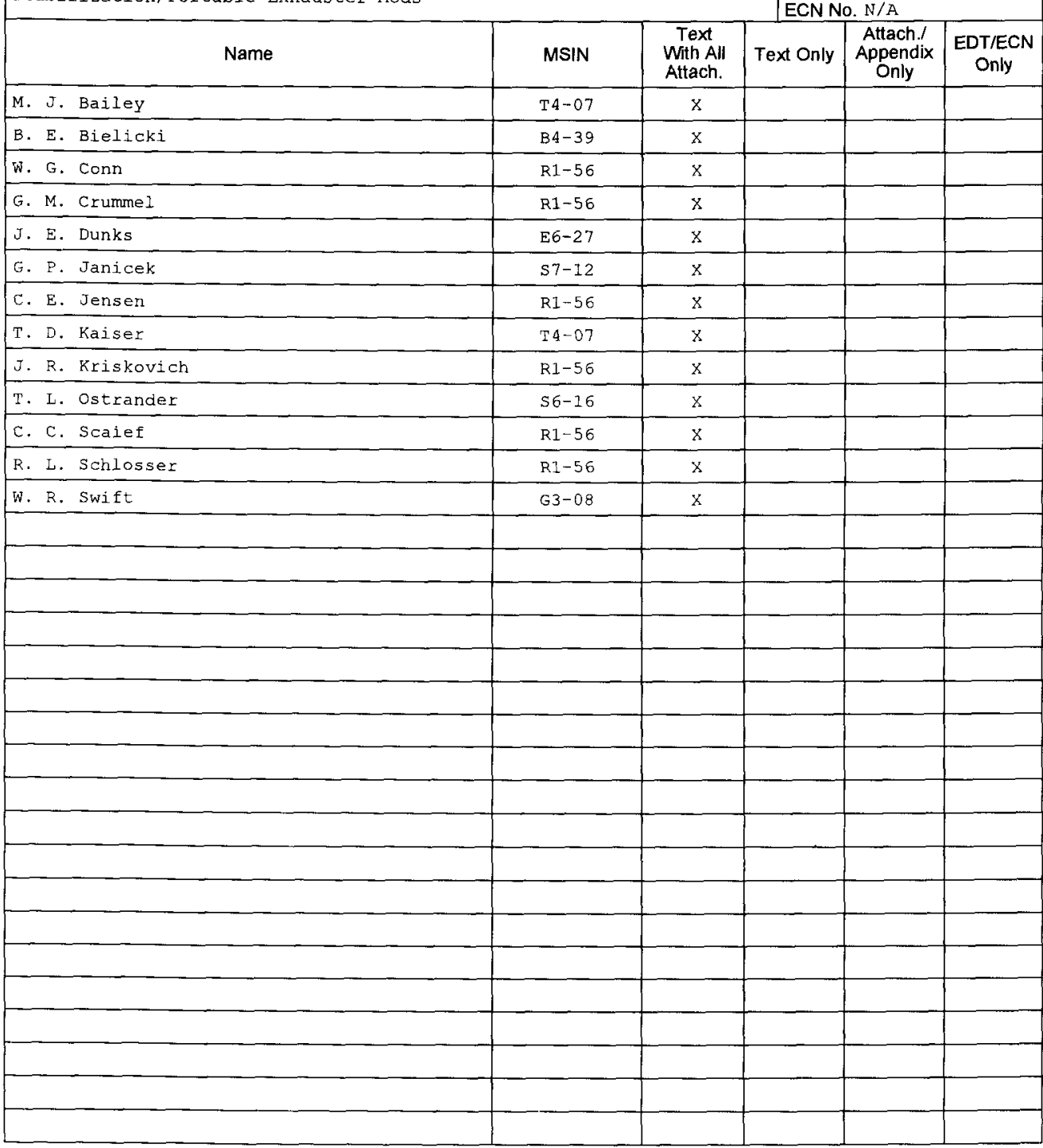

From

B.E. Bielicki, FDNW, 454
Page 1 of 1

Date $12 / 21 / 98$

EDT No. 620994

ECN No. N/A 